\title{
Three dimensional co-registration between a positron emission tracking system and a C-arm $\mathrm{x}$-ray imaging system
}

by

Benjamin A. Spencer

A Thesis submitted to

the Faculty of Graduate \& Post Doctoral Affairs

in partial fulfilment of

the requirements for the degree of

Master of Science

Ottawa-Carleton Institute of Physics

\author{
Department of Physics \\ Carleton University \\ Ottawa, Ontario, Canada
}

September 2012

Copyright (C)

2012 - Benjamin A. Spencer 
Library and Archives

Canada

Published Heritage

Branch

395 Wellington Street

Ottawa ON K1A ON4

Canada
Bibliothèque et

Archives Canada

Direction du

Patrimoine de l'édition

395 , rue Wellington

Ottawa ON K1A ON4

Canada
Your file Votre référence

ISBN: 978-0-494-93546-0

Our file Notre référence

ISBN: $978-0-494-93546-0$
NOTICE:

The author has granted a nonexclusive license allowing Library and Archives Canada to reproduce, publish, archive, preserve, conserve, communicate to the public by telecommunication or on the Internet, loan, distrbute and sell theses worldwide, for commercial or noncommercial purposes, in microform, paper, electronic and/or any other formats.

The author retains copyright ownership and moral rights in this thesis. Neither the thesis nor substantial extracts from it may be printed or otherwise reproduced without the author's permission.
AVIS:

L'auteur a accordé une licence non exclusive permettant à la Bibliothèque et Archives Canada de reproduire, publier, archiver, sauvegarder, conserver, transmettre au public par télécommunication ou par l'Internet, prêter, distribuer et vendre des thèses partout dans le monde, à des fins commerciales ou autres, sur support microforme, papier, électronique et/ou autres formats.

L'auteur conserve la propriété du droit d'auteur et des droits moraux qui protege cette thèse. $\mathrm{Ni}$ la thèse ni des extraits substantiels de celle-ci ne doivent être imprimés ou autrement reproduits sans son autorisation.
In compliance with the Canadian Privacy Act some supporting forms may have been removed from this thesis.

While these forms may be included in the document page count, their removal does not represent any loss of content from the thesis.
Conformément à la loi canadienne sur la protection de la vie privée, quelques formulaires secondaires ont été enlevés de cette thèse.

Bien que ces formulaires aient inclus dans la pagination, il n'y aura aucun contenu manquant. 


\section{Abstract}

Real-time motion tracking is required for accurate delivery of radiation therapy to tumours undergoing motion due to respiration, as well as for the precise guidance, manipulation, and operation of surgical tools or devices used during non-invasive interventional procedures. Positron emission real-time three-dimensional (3D) tracking (PeTrack) is a proposed solution to these problems which is currently being developed at Carleton University. The technique involves the localization and tracking of positron emission fiducial markers which could be implanted into a tumour volume or integrated into a surgical tool or instrument. The research presented here describes the co-registration of the PeTrack localization and tracking system with a $\mathrm{C}$-arm $\mathrm{x}$ ray imaging system capable of $3 \mathrm{D}$ cone-beam imaging. This co-registration allows the display of objects tracked in 3D by the PeTrack system on to a 3D reconstructed image.

The acquisition of accurate 3D images from the $\mathrm{x}$-ray imaging system requires: $\mathrm{x}$-ray detector distortion correction, geometric calibration of the C-arm $\mathrm{x}$-ray scanner, and then a method to reconstruct 3D images. PeTrack localization and tracking of positron sources requires a detector system capable of detecting positron sources, and an algorithm which can localize and track positron sources based on the information provided by the detector system. This tracking algorithm has previously been developed. The co-registration of the $\mathrm{x}$-ray imaging system and the PeTrack tracking system requires the geometric calibrations of the PeTrack and the $\mathrm{x}$-ray imaging 
systems both defined in a common 3D reference frame.

The results presented in this work show that distortion correction is essential for the acquisition of high quality 3D image reconstructions. The method of $x$-ray scanner geometric calibration implemented was validated for the the first time with real data. A method of PeTrack geometric calibration was developed and evaluated. A simulation study showed that under ideal conditions the calibration performs exceptionally well. The $\mathrm{x}$-ray and PeTrack geometric calibration were used along with the dual calibration phantom to successfully co-register the two systems. Evaluations of the co-registration exhibited an error of $1.8 \pm 0.2 \mathrm{~mm}$ between the location of sources identified on an image and their locations projected on to the image using the two geometric calibrations. A few sources of systematic error are discussed and subject to further investigation. 
I would like to dedicate this thesis to:

my undergraduate lab coordinator Penka Matanska

my supervisor Dr. Tong $\mathrm{Xu}$

and my friend and research collaborator Dr. Rolf Clackdoyle 


\section{Acknowledgments}

I would like to acknowledge the collaborators that made this research possible:

Dr. Rolf Clackdoyle for the development of the x-ray geometric calibration method and the best circle fitting process and for his essential assistance programming and implementing the $\mathrm{x}$-ray geometric calibration method and the process of generating three-dimensional(3D) image reconstructions,

Dr. Catherinne Mennessier for her work developing and programming the x-ray geometric calibration method and best circle fitting,

Marc Chamberland for the development of the PeTrack tracking algorithm and the GATE simulation data,

Simon Massad for his work on the detector development and calibration, and my supervisor Dr. Tong Xu. 


\section{Statement of Originality}

The work presented here contains contributions from several sources other than myself and my supervisor Dr. Tong Xu.

The distortion correction main algorithm was not new however I implemented and adapted it along with several other steps to accomplish the angle dependent image intensifier distortion correction.

The method of x-ray geometric calibration had previously been developed and published by Dr. Catherine Mennessier and Dr. Rolf Clackdoyle in [1]. The algorithm had also been programed by Dr. Mennessier and independently programed by Dr. Rolf Clackdoyle and I. The x-ray calibration phantom was uniquely designed and constructed by Dr. Tong Xu, Dr. Rolf Clackdoyle and myself.

The reconstruction algorithm as well as the program used to do image reconstruction had been previously developed and written. My contribution involved implementation of this program and the analysis and evaluation of the generated image reconstructions.

The PeTrack geometric calibration and the PeTrack/x-ray imaging system coregistration along with all the evaluations was completed by me. This work marked the first time a method of geometric calibration and co-registration has been developed for a positron emission based real-time tracking system. The construction of the PeTrack prototype was done in consultation with $\mathrm{Dr}$. Tong $\mathrm{Xu}$, by myself with help also from Marc Chamberland. 
This work has in part been presented at the 2011 IEE MIC conference in Valencia, Spain [2], and the CT meeting conference in Salt Lake City, Utah [3]. 


\section{Table of Contents}

$\begin{array}{ll}\text { Abstract } & \text { ii }\end{array}$

Acknowledgments $\quad$ v

Preface vi vi

Table of Contents viii

List of Tables $\quad$ xii

List of Figures xiii

Nomenclature $\quad$ xvi

1 Introduction 1

1.1 Real-time motion tracking . . . . . . . . . . . . . . . 1

1.1.1 Motion tracking in medicine . . . . . . . . . . . . . 1

1.1.2 PeTrack: a technique for real-time 3D motion tracking . . . . 6

1.1.3 PeTrack development to date ... . . . . . . . . . . 8

1.2 PeTrack project goals in this study . . . . . . . . . . . 9

1.3 PeTrack detector and $\mathrm{x}$-ray imaging devices . . . . . . . . . . . . 12

1.3.1 PeTrack detector system . . . . . . . . . . . . . . 12

1.3.2 C-arm for 3D imaging . . . . . . . . . . . . . 13 
1.3.3 The image intensifier (II) detector . . . . . . . . . 15

1.4 Summary . . . . . . . . . . . . . . . . . . . . 16

2 Distortion: Measurement \& Correction 18

2.1 Overview . . . . . . . . . . . . . . . . . . 18

2.1 .1 Distortion . . . . . . . . . . . . . . . . . . 19

2.1.2 Image intensifier distortion correction in the literature . . . . 21

2.1.3 Image intensifier pin-cushion distortion correction from the Siemens on-board system . . . . . . . . . . . . . . 23

2.1.4 Implemented method of distortion correction . . . . . . . . 24

2.2 Distortion measurement and correction procedure . . . . . . . 25

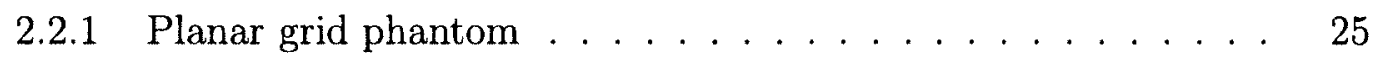

2.2 .2 Distortion measurement $\ldots \ldots \ldots \ldots$

2.2 .3 Ideal undistorted estimation . . . . . . . . . . . 27

2.2.4 Regional distortion correction: parametrization . . . . . 28

2.2 .5 Regions at the edge of the field of view . . . . . . . . 30

2.2 .6 Image correction $\ldots \ldots \ldots \ldots \ldots$

2.2.7 Distortion measurement \& correction procedure overview . . . 32

2.3 Distortion correction evaluation $\ldots \ldots \ldots \ldots \ldots$

2.3.1 Evaluation for a single projection image of the grid . . . . 33

2.3.2 Degree of distortion during a full scan $\ldots \ldots \ldots$. . . . . 34

2.4 Discussion . . . . . . . . . . . . . . . . . . . . . 36

3 Scanner Geometry Calibration 38

3.1 Overview . . . . . . . . . . . . . . . . . . 38

3.1.1 Siemens geometric calibration method . . . . . . . . . 39

3.1.2 Purpose for off-line geometric calibration . . . . . . . . 40

3.2 Geometric calibration method . . . . . . . . . . . . . . 41 
3.2 .1 Geometric calibration phantom $\ldots \ldots \ldots 42$

3.2.2 Mathematical formalism of geometric calibration . . . . . . 43

3.3 Geometric calibration implementation . . . . . . . . . . . . . . 47

3.3 .1 Overview . . . . . . . . . . . . . . . . 49

3.4 Results . . . . . . . . . . . . . . . . . . . . . . 49

3.4.1 Measurements of the calibration phantom . . . . . . 49

3.4.2 Evaluation with and without distortion correction . . . . . 50

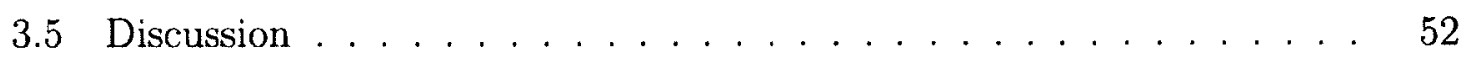

3.5 .1 Summary . . . . . . . . . . . . . . 54

4 Image Reconstruction and Evaluation $\quad 55$

4.1 Method . . . . . . . . . . . . . . . . . 56

4.1 .1 Image reconstruction theory $\ldots \ldots \ldots \ldots$

4.1.2 Re-expression of geometric calibration parameters . . . . . 58

4.1 .3 Reconstruction programs . . . . . . . . . . . . . . . 59

4.2 Reconstruction method application . . . . . . . . . . . 60

4.2.1 Image formatting \& pre-processing $\ldots \ldots \ldots \ldots$

4.2 .2 Orbit file generation $\ldots \ldots \ldots \ldots \ldots \ldots$

4.3 Evaluations . . . . . . . . . . . . . . . . . 62

4.4 Discussion . . . . . . . . . . . . . . . . 67

5 PeTrack: Prototype construction, Calibration \& Co-registration 70

5.1 Prototype construction . . . . . . . . . . . . 70

5.1 .1 Prototype assembly . . . . . . . . . . . . . 70

5.1 .2 Data acquisition. . . . . . . . . . . . . 73

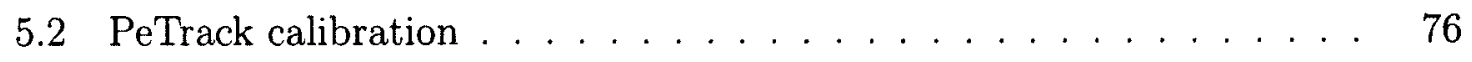

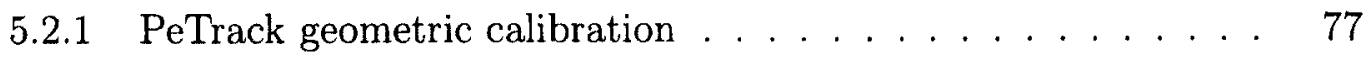

5.2 .2 Implementation $\ldots \ldots \ldots \ldots \ldots \ldots \ldots$ 
5.2.3 Calibration simulation ............... 82

5.3 PeTrack co-registration . . . . . . . . . . . . . . 85

5.3.1 Dual x-ray/PeTrack calibration phantom . . . . . . . . 85

5.3 .2 Co-registration method . . . . . . . . . . 87

5.3.3 Co-registration evaluation ............... 89

5.4 Discussion . . . . . . . . . . . . . . . . . . 93

5.4 .1 Summary ............................ 94

6 Conclusions \& Future Work 96

6.1 Distortion measurement \& correction . . . . . . . . . . . . . . . 96

6.2 Scanner geometry calibration . . . . . . . . . . . . . . . 97

6.3 Image reconstruction . . . . . . . . . . . . . . . . . . 97

6.4 PeTrack calibration \& co-registration . . . . . . . . . . . . . . . . . 98

6.5 Project summary . . . . . . . . . . . . . . . . . . 99

$\begin{array}{ll}\text { List of References } & 100\end{array}$ 


\section{List of Tables}

3.1 X-ray calibration phantom measurements. . . . . . . . . . 50

3.2 Informal comparison of the listed and average values of the focal length and source to isocenter distance. . . . . . . . . . . . . . . . 52

5.1 True and simulated source locations after rigid translation and rotation. 83

5.2 PeTrack localization of simulated source locations and the determination and application of a rigid translation and rotation calibration. . . 84

5.3 Measurements of the x-ray markers exterior ball to ball length . . . 87

5.4 Positron marker $k$ values calculated based on the $\mathrm{x}$-ray marker measurements and source housing length. . . . . . . . . . . . 88

5.5 Comparison between the projected $(u, v)$ source locations (determined from the tracking and co-registration) and the estimated source locations in the image $\ldots \ldots \ldots \ldots \ldots \ldots \ldots \ldots$

5.6 PeTrack tracking precision. The average and standard deviation of 30 measurements of the $(x, y, z)$ location of four tracked sources. . . . . 92 


\section{List of Figures}

1.1 An example of a positron annihilation event. . . . . . . . . 6

1.2 The research steps of this project illustrated in a flow chart. . . . . 11

1.3 Schematic diagram of the PeTrack system. . . . . . . . . . . . 13

1.4 Siemens Arcadis Orbic C-arm x-ray system. . . . . . . . . . . 14

1.5 Schematic of the image intensifier (II) and II input window. . . . . 17

2.1 An example of the effects of pin-cushion distortion . . . . . . . 20

2.2 The effects of magnetic fields on electrons traveling within the II. . . 21

2.3 An example of the effects of S-distortion . . . . . . . . . 21

2.4 Planar grid phantom used for distortion correction. . . . . . . . . 26

2.5 Grid phantom imaged directly on the II. . . . . . . . . . . 26

2.6 Mapping of each point from the ideal image to the distorted image. . 29

2.7 An example of triangular regions in which one or more balls are outside of the field of view. . . . . . . . . . . . . . . 30

2.8 Parameterization of regions with two or less balls in the image FOV. 31

2.9 Illustrative example of the process of gray level bilinear interpolation. 32

2.10 Illustration of uncorrected distortion and an indication of the fidelity

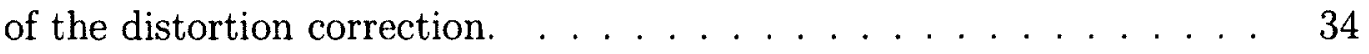

2.11 Distorted ball locations plotted on corrected projection image \#50 to illustrate the magnitude of the distortion. . . . . . . . . . 35

3.1 Siemens geometric calibration phantom. . . . . . . . . . . 40 
3.2 Geometric calibration parameters labeled on C-arm. . . . . . . . . . . 42

3.3 X-ray geometric calibration phantom. . . . . . . . . . . . . 43

3.4 Projection of a point in space onto the detector with relevant geometric parameters indicated. . . . . . . . . . . . . . 45

3.5 One of the projection images of the geometric calibration phantom suspended in a plastic cylindrical case. . . . . . . . . . . . . . . . 48

3.6 Plots of geometric calibration parameters over projection index with and without distortion correction. . . . . . . . . . . . 51

4.1 Diagram representing the source trajectory locations and the "best circle" determination of the source trajectory frame. . . . . . . . . . . 59

4.2 Volume view of a high resolution reconstruction of the geometric calibration phantom. ................... 63

$4.3 \mathrm{x}$ direction slice view of the reconstructed geometric calibration phantom. 64

$4.4 \mathrm{x}$ direction slice view of the reconstructed resolution phantom. . . . . 64

4.5 Profile plot of the calibration phantom. . . . . . . . . . . . 65

4.6 Profile plot of the resolution grid phantom. . . . . . . . . . . . . 66

$4.7 \mathrm{z}$ direction slice view of quality phantom. . . . . . . . . . . . 66

4.8 Hand phantom composed of bone encased in Lucite. . . . . . . . . . . 67

4.9 Volume view and $\mathrm{x}$ direction slice view of the reconstruction of the hand phantom. ..................... 69

5.1 Various steps of PMT construction. . . . . . . . . . . . . . . 72

5.2 Detector module mounted on frame. . . . . . . . . . . . . . 73

5.3 PeTrack detectors and structure. . . . . . . . . . . . . . . . . 74

5.4 The MPET-8 and MCPD2 electronics. . . . . . . . . . . . . . 75

5.5 Illustration of the PeTrack coordinate frame. . . . . . . . . . . . 77

5.6 Illustration of the calibration phantom design. . . . . . . . . . . . . . 80

5.7 Schematic of the dual calibration phantom design. . . . . . . . . . . . 86 


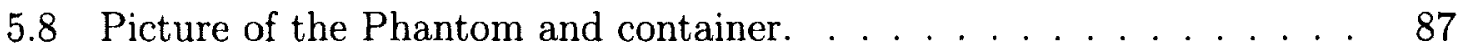

5.9 Projection image of the dual calibration phantom. . . . . . . 88

5.10 The projected location of tracked sources onto a projection image. . . 90

5.11 Example of x-ray markers visually observed on projection image. . . . 91 


\section{List of Abbreviations}

\begin{tabular}{|c|c|}
\hline $2 \mathrm{D}$ & Two dimensional \\
\hline $3 \mathrm{D}$ & Three dimensional \\
\hline $\mathrm{BGO}$ & Bismuth germinate $\left(\mathrm{Bi}_{4} \mathrm{Ge}_{3} \mathrm{O}_{12}\right)$ \\
\hline $\mathrm{CT}$ & Computed tomography \\
\hline $\mathrm{EM}$ & Electromagnetic \\
\hline FOV & Field of view \\
\hline II & Image intensifier \\
\hline IP & Interventional procedures \\
\hline Linac & Linear accelerator \\
\hline PeTrack & Positron emission real-time tracking \\
\hline PMT & Photo-multiplier tube \\
\hline SPECT & Single photon emission computed tomography \\
\hline US & Ultrasound \\
\hline XRT & X-ray tube \\
\hline
\end{tabular}




\section{Chapter 1}

\section{Introduction}

\section{$1.1 \quad$ Real-time motion tracking}

\subsubsection{Motion tracking in medicine}

There are many applications in medicine for the real-time motion tracking of an object to determine its position, orientation or possibly even shape. The application of real-time tracking of objects such as organs, tumours, endoscopes, and catheters is useful in medicine. Some examples of the necessity for motion tracking in medicine are discussed below.

Tracking of organs undergoing motion due to patient breathing is useful during tomographic imaging. The tracked organ motion during image acquisition can be accounted for in the image to create a $4 \mathrm{D}$ motion compensated image. Real-time instrument and tool tracking is useful for the precise operation and manipulation of tools during minimally invasive interventional procedures (IP). Additionally, and possibly most importantly, real-time tumour tracking is essential for the accurate delivery of radiation therapy to tumours which are undergoing motion due to patient breathing. The current techniques of motion tracking for the accurate delivery of radiation treatment and for interventional procedures (IP) will be described below. 


\section{Interventional procedures: tool and device tracking}

Many medical procedures and forms of treatment can be accomplished by the insertion of needles or small instruments into the skin, through blood vessel or possibly along the spine; such procedures are termed interventional procedures (IP). This allows access to the area under investigation or being treated in a minimally invasive way, which is quite favorable compared to the invasive alternative, a full surgical procedure. The tools and devices used need to be guided and monitored inside the patient during the procedure to ensure precise manipulation and correct operation. Currently this can be done in two ways: by using an imaging modality (such as ultrasound (US) or x-ray fluoroscopy) to see the instrument inside the patient, or by using markers placed on the instrument to track it in 3D while inside the patient.

One of the most common methods of instrument tracking for IP is tracking through $\mathrm{x}$-ray imaging. Guidance and monitoring of tools by $\mathrm{x}$-ray fluoroscopy imaging results in a significant amount of dose being delivered to both the patient and operator. The dose delivered becomes increasingly excessive when the procedure is complex. Ultrasound (US) imaging for tracking suffers from a limited ability to image the instrument in cases when the instrument is near certain types of tissue. For instance, US imaging degrades when trying to image objects in the vicinity of bone or lung.

The tracking of objects for IP can also be done through optical tracking, ultrasound tracking, and electromagnetic (EM) tracking. All of these tracking techniques have some advantages and disadvantages. Optical tracking simply uses video cameras to visually measure the movement of the instruments by tracking insignia (such as reflective tape or LED lights) marked on the outside of the instrument [4]. Based on the recorded movement of the insignia the position of the instrument in the patient is inferred [4]. Ultrasound tracking (as opposed to imaging)is accomplished by placing small US transmitters on the outside of the instrument, possibly attached near the 
handle. While the instrument is in use the generated US signal is measured using a system of microphones which is fixed in place in the room close to the patient [5]. This method of tracking is a passive method which only infers where the instrument is based on the movement of the handle. Both of these passive methods, optical and US tracking, are not adequate for non-rigid, maneuverable tools. They are only useful for procedures using rigid tools such as biopsies [4].

An accurate tracking technique in current use tracks the motion of instruments used for IP using an EM field generator and a miniature sensor coil placed in the instrument(eg. in a needle) tip. The EM field generator produces a varying magnetic field which passes through the patient and induces a signal in the sensor coil. The signal induced in the wired sensor coil can be measured to accurately indicate the 3D position and orientation of the instrument [6]. This technique has the disadvantage that the sensor coil must be wired and, along with all EM techniques, that the accuracy of the position and orientation of the sensor can suffer from a great deal of interference from metallic objects which are regularly used in the operating room [7-9].

The necessity for a reliable, versatile, accurate, and low dose method of real-time instrument and tool tracking during IP is apparent.

\section{Tumour tracking for radiation therapy}

External beam radiation therapy is one of the principle means of treating cancerous tumours. Current technology allows the very accurate delivery of radiation to the desired target volume of a tumour, however, tumours in the lungs or abdomen are constantly undergoing motion due to patient breathing. As a result, the accurate delivery of radiation therapy to tumors undergoing motion is often unrealistic, resulting in either an inadequate dose being delivered to the tumour or, and possibly as well as, an unacceptable amount of dose being delivered to healthy tissue. Accurate delivery 
of radiation therapy to tumours undergoing motion can be achieved through respiratory gated radiation therapy. This technique, however, requires knowledge of the exact location of the tumour in real-time; hence the necessity for real-time tracking.

There are two methods of tracking tumour motion: tracking of fiducial markers implanted in the tumour volume, or tracking of external markers placed on the patient's skin and estimating tumour motion by correlating the external marker motion with tumour motion. It has been shown both that external markers may not accurately characterize tumour motion and that the external marker to tumour correlation may change between treatment sessions resulting in an erroneous tracking technique [10,11]. External marker motion tracking may still be utilized with patients who cannot receive implantation of markers [11].

Methods of tracking using implanted markers include: $\mathrm{x}$-ray fluoroscopy of radio opaque markers, electromagnetic tracking using transponders, and a more recent technique of tracking using single photon emission from a radioactive source marker. Implanted radio opaque markers tracked by x-ray fluoroscopy can accurately track tumour motion in real-time $[12,13]$, however, this technique has a majour disadvantage. The constant $\mathrm{x}$-ray fluoroscopy needed for tracking delivers a significant amount of dose to the skin (possibly exceeding $1 \mathrm{~Gy} /$ hour of treatment) as well as dose to other healthy tissue [14].

Electromagnetic (EM) tracking uses a coil array to track implanted EM transponders. The array contains both source coils and receiver coils. The source coils generate an EM field which is induced and resonates in the transducers, then once the source coils are turned off the transducer EM field resonance is measured by the receiver coils to determine the transducers 3D location [15]. Although this technique delivers no ionizing radiation dose to the patient it still requires implantation of markers of impractical size [16] especially in the case of lung implantations and can cause serious problems such as pneumothorax (a collapsed lung) [17]. Additionally, the accuracy of 
the 3D tracking can be disrupted by electromagnetic interference [16], and there are majour artifacts produced by the transponders when imaging patients with magnetic resonance imaging (MRI) [18].

There is a more recent technique of positioning and tracking using an implanted radioactive source which is now in clinical use. The method was developed by NavoTek originally to be used for positioning of prostate cancer patients before radiation therapy treatment but can be extended to localization and motion tracking in organs such as the liver or lung $[19,20]$. A single radioactive source marker is implanted into the tumour and a series of 3 detectors independently measure the angle corresponding to the strongest source signal. Each detector determines a plane in which the source must lie; the intersection of the 3 planes determines the real-time $3 \mathrm{D}$ position of the source with a high degree of accuracy [19]. Further, the detector system is small and can be easily mounted on a linear accelerator (Linac) gantry. The implanted radioactive marker is very small, only 240 microns, which greatly facilitates complication free implantation. The marker used is an iridium (Ir-192) source with a half life of 74 days. Though only a small activity, $100 \mu \mathrm{Ci}$, is needed for localization, due to the long source half-life, long term dose affects should be considered [21]. The majour disadvantage of this method is that it cannot track tumour movement during radiation treatment using a beam energy of $10 \mathrm{MV}$ or above [19]. The high-energy beam creates many short lived radioactive isotopes which make the small source strength of the marker relatively invisible. At $10 \mathrm{MV}$ beam energy the source can be located immediately after irradiation, however, if the beam energy is at $15 \mathrm{MV}$ the source marker cannot be localized for tens of seconds afterward [19]. During irradiation with a beam energy of $6 \mathrm{MV}$, tracking can occur with some localization errors [19].

Though the above techniques offer well developed, suitable solutions of real-time 3D tracking they all possess limitations. The requirement of a method for real-time tumour tracking that: is accurate; uses very small implanted markers (if at all); does 
not involve significant radiation exposure to healthy tissue; and can be performed during radiation therapy; is essential in medicine.

\subsubsection{PeTrack: a technique for real-time 3D motion tracking}

The research presented in this thesis will be directed toward the development of a method of real-time motion tracking using implanted positron emission markers, called PeTrack [22]. The proposed technique provides a method of tracking which uses very small $(0.5-0.8 \mathrm{~mm})$ implanted fiducial markers, administers very little dose to the patient or operator, can effectively track during linear accelerator (Linac) operation, and has a proposed tracking accuracy on the order of $0.5 \mathrm{~mm}$.

The tracking uses positron emission sources as markers. These radioactive sources decay naturally releasing positrons which annihilate with electrons close to the location of their release. When the positron annihilates with an electron they create two $511 \mathrm{keV}$ photons which are emitted in opposite directions, (see Fig. 1.1). Provided both of these photons are detected they will be detected at close to the same time; this is called a coincidence event. The event then indicates that the annihilation occurred somewhere along the line joining the two detection locations. This is the main principle behind positron emission tracking and it has been implemented for imaging in positron emission tomography (PET) for many years [23].

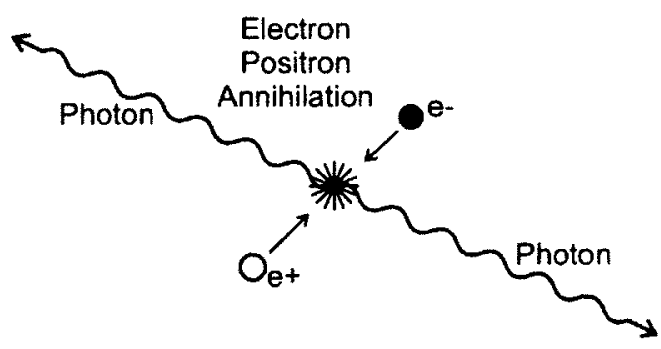

Figure 1.1: An example of a positron annihilation event. 
Unlike PET which uses a full $360^{\circ}$ ring of detectors, our system uses only two pairs of position sensitive gamma detectors to detect the coincidence events [23], (see Fig. 1.3), described in more detail in section 1.3.1. The PeTrack system would be light weight, small, and mobile so it can be easily added to existing systems for the incorporation of object tracking. In theory, by using this system accurate real-time 3D tracking of an object can be accomplished by the implantation of small, low activity positron markers.

In the case of interventional procedures (IP) markers would be placed in the handle of the tool or instrument requiring tracking. Using multiple markers the tool's location and orientation in 3D space could be accurately determined. The tracking would not require constant imaging of the patient, would deliver a minuscule dose to the patient and operator, and the tracking accuracy would not be affected by any tools or objects in the vicinity. The PeTrack localization system would have to be coregistered with an imaging system's coordinates so that the location of the instrument or tool could be displayed on the patient image in real-time to accurately visualize where the instrument is relative to the patients anatomy. This implementation would be a great advancement for IP and could later be applied to other areas of medicine.

PeTrack could be easily applied to real-time tumour tracking during radiation therapy. Up to three low activity, small, positron markers would be implanted in the tumour using a needle similar to those used for biopsy or brachytherapy. The positron source selected would be one with a half-life on the order of the treatment time with the purpose that the source will quickly decay over time and provide little dose to the patient once treatment has been completed. The lightweight and relatively small PeTrack detector system could be easily integrated into the Linac treatment room. The PeTrack coordinate system would have to be co-registered with the Linac system in order for the PeTrack localization and tracking to be accurately known on the Linac image-guided therapy system. Consequently accurate real-time tumour 
motion tracking and gated radiation therapy would be permitted; the ramifications of real-time tumour tracking for gated radiation therapy would be prevalent in medicine.

\subsubsection{PeTrack development to date}

In 2006 Tong $\mathrm{Xu}$ et al. [22] first envisioned and analyzed the PeTrack concept. The method was proposed as a real-time tumour tracking technique capable of submillimeter tracking accuracy using $(0.5-0.8 \mathrm{~mm})$ diameter positron markers which are about one half the size of markers currently employed by most other techniques. The markers would be labeled with low activity positron sources with half lives on the order of days too weeks, such as iodine-124 (I-124), arsenic-74 (As-74), or Rubidium$84(\mathrm{Rb}-84)$. This ensures the radioactive sources promptly decay to zero activity reducing the amount of lifetime dose delivered to the patient. A significant advancement was the development of an algorithm to localize and track multiple positron emission markers; an expectation-maximization clustering algorithm. Additionally, a Monte Carlo simulation model was developed to test the algorithm and concept of PeTrack. Through simulation studies it was shown that the PeTrack technique could potentially provide real-time tracking using low dose and physically small positron markers.

Further work was discussed in Churchill, Chamberland and Xu [24]. In this work a positron emission line source was proposed as an improved and more favourable means of $3 \mathrm{D}$ tracking rather than the implantation of multiple point sources. A positron emission localization algorithm for tracking line sources was developed and tested. The Monte Carlo simulation package GATE [25] was utilized to show that a line source marker mid-point and angle could be determined very accurately using the developed tracking algorithm. 
In 2011 Chamberland et al. [26] further developed the positron source tracking algorithm before preforming phantom testing. The algorithm was expanded with methods of: initialization, failure distinction of initialization, and then re-initialization in the event of failure. Another significant improvement to the algorithm was the capability of marker prediction during tracking. The tracking algorithm was analyzed based on its ability not only to track motion in real-time but predict marker location 100 - $200 \mathrm{~ms}$ in advance. A clinical PET machine was utilized to detect a marker undergoing simulated breathing motion within a dynamic thorax phantom. For a latency of $100 \mathrm{~ms}$ the $3 \mathrm{D}$ predictive error was $1.3 \pm 0.6 \mathrm{~mm}$, while for $200 \mathrm{~ms}$ the $3 \mathrm{D}$ predictive error was $1.4 \pm 0.7 \mathrm{~mm}$.

A great deal of research has been accomplished on the PeTrack project from the concept to the present. Much more research still needs to be done before the construction of a working prototype. Some of this research will be presented and discussed in this thesis.

\subsection{PeTrack project goals in this study}

The research presented here is directed toward the construction of a full scale PeTrack system prototype which has the ability to track the movement of multiple markers and accurately display their locations on a $2 \mathrm{D}$ or $3 \mathrm{D}$ image. The algorithm and interface for tracking multiple sources has already been developed and tested. Further, a two detector small scale example of the system has been tested. These aspects of the project will not be discussed but are discussed in detail in [26] for information about the tracking algorithm and $[27,28]$ for detector specific information.

The first step in this project is the construction of a full scale PeTrack detector system (described in chapter 5). As described above the detector system contains 
two pairs of detector modules mounted on a lightweight frame allowing for maneuverability.

Next the calibration of the PeTrack system must be achieved to accurately know where the positron emission markers are in 3D space, (see chapter 5). This is performed by using a known calibration phantom to measure the detector locations relative to the phantom frame. Once the detector locations are accurately known in the phantom frame any subsequent source locations determined will also be accurately known in the phantom frame.

The PeTrack system is then integrated with an imaging system capable of 3D image generation in order to display the tracked source locations on either $2 \mathrm{D}$ or 3D images (see Fig. 1.2). Such an imaging system could be a C-arm x-ray imaging machine capable of $2 \mathrm{D}$ image acquisition during gantry rotation and consequently $3 \mathrm{D}$ image reconstruction (see section 1.3.2).

In order to accurately display the source locations on an acquired image, coregistration between the PeTrack source location coordinate system and the imaging coordinate system is necessary (see section 5.3). Otherwise, for example, it would be impossible to know whether a $1 \mathrm{~cm}$ movement in the $\mathrm{x}$-direction of a tracked source corresponds to a $1 \mathrm{~cm}$ movement in the $\mathrm{x}$-direction or a $3 \mathrm{~cm}$ movement in the $y$-direction on the image. To co-register the two systems requires an object that describes a common reference frame for both systems, a dual imaging and PeTrack calibration phantom is a way to execute this. The phantom design should acquire the necessary information to calibrate the PeTrack system, as well as the necessary information to accurately define the imaging system. A method to calibrate the imaging system is required to know how the image is produced relative to the calibration object which also defines the relative movement of the tracked source locations. These two calibrations performed on the same phantom co-register the image with the PeTrack localization and tracking. 
Imaging System

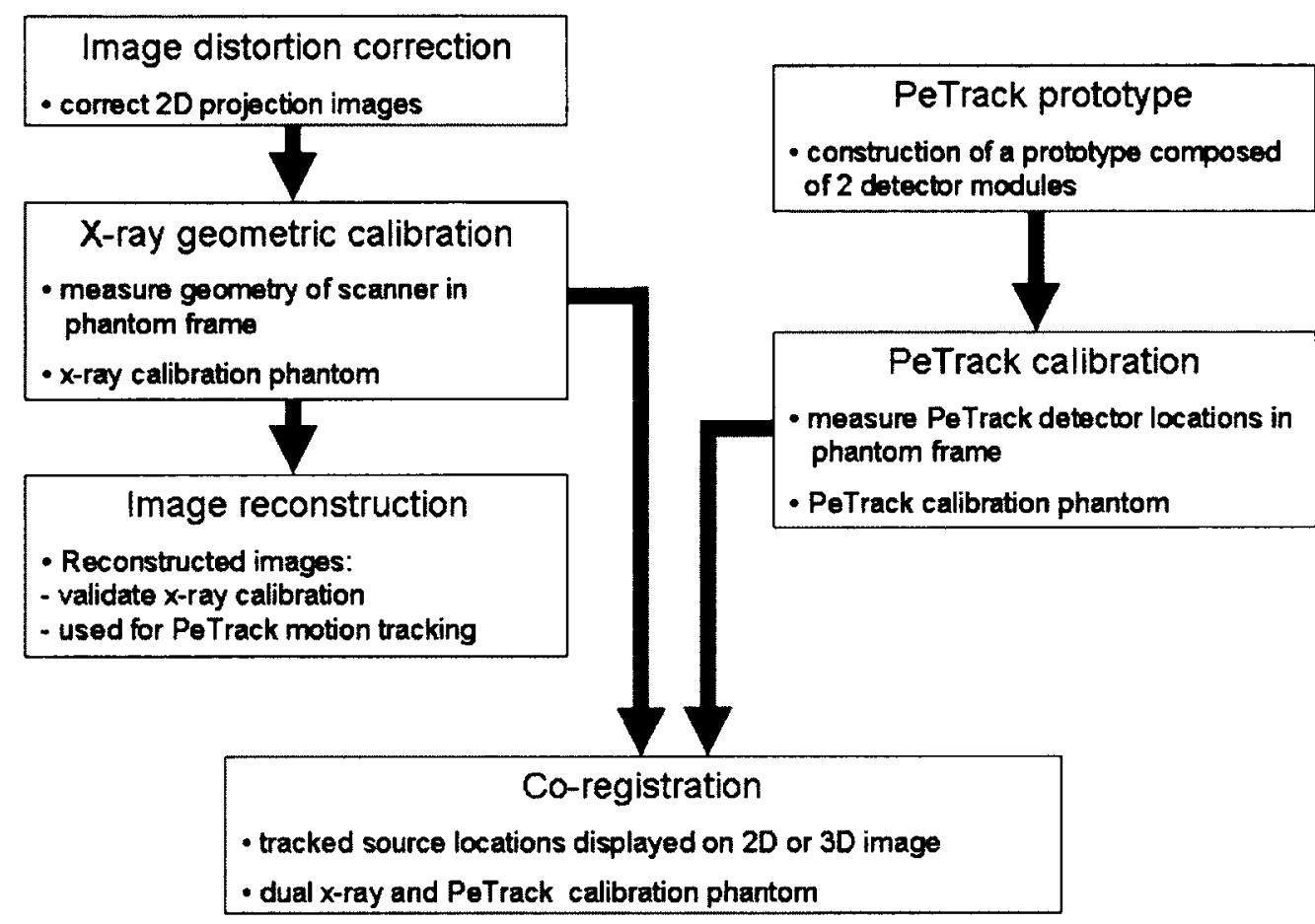

Figure 1.2: The research steps of this project illustrated in a flow chart.

Calibration of the imaging system entails exact measurement of the source and detector pair to describe how the projection of an object at a specific location would be observed by the detector, and in the 2D image (described in Chapter 3). Further, the imaging system calibration provides enough information to generate 3D reconstructions of acquired 2D image data sets using a simple analytic short-scan method (see chapter 4).

In order to ensure the accurate generation of a 3D image, the $2 \mathrm{D}$ projection data acquired must be displayed exactly. For many imaging systems this is not the case due to inherent sources of image distortion. In this case image distortion measurement and correction is essential before data acquisition (see chapter 2).

Implementation of the above steps would bring about multiple positron emission 
marker localization and tracking co-registered with accurate 2D or 3D image coordinates. Once this is completed it is theoretically possible, though not in real-time, to do tracking of instruments used for interventional procedures. This sets the stage for the integration of the PeTrack system with other systems such as a Linac to facilitate real-time image-guided gated radiation therapy.

\subsection{PeTrack detector and x-ray imaging devices}

\subsubsection{PeTrack detector system}

The positron emission detector system consists of 2 pairs of gamma ray photon detectors. Each pair of gamma detectors is comprised of 2 detector modules which face each other through the PeTrack system field of view (FOV) (see Fig. 1.3). The modules face each other to detect the positron emission annihilation events. The annihilation creates two $511 \mathrm{keV}$ photons traveling back-to-back from one another and if they are going in the correct direction each module pair may detect one of the two photons of the coincidence event.

Each detector module is composed of 4 scintillation crystal photomultiplier tube (PMT) detectors. The scintillation material is bismuth germinate $\left(\mathrm{Bi}_{4} \mathrm{Ge}_{3} \mathrm{O}_{12}\right)$ or BGO for short. BGO has been shown to be an exceptional scintillation material for gamma ray photons with $511 \mathrm{keV}$ energy, the energy of photons emitted from positron annihilation [29]. BGO scintillation material has both a very high density and a high atomic number which is optimal for interaction with high-energy photons. The scintillation material is arranged in a $12 \times 12$ array of crystals with a thickness of $2 \mathrm{~cm}$. When a high-energy gamma ray photon enters the scintillation material it interacts with the material by converting its energy to produce many lower energy visible light photons which travel down the length of the crystal to be detected by 


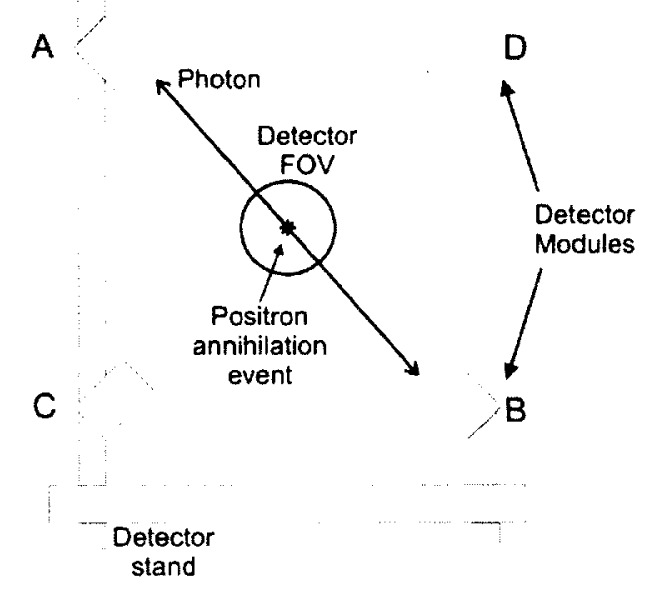

Figure 1.3: Schematic diagram of the PeTrack system.

the PMT. The PMT collects and amplifies the signal from the visible light photons produced in the crystal. A signal pre-amplifier is also part of the detector design, which along with the PMT and BGO scintillation material is all sealed inside a light tight detector housing, and placed into the detector module.

The PMT detector readout is accomplished by the "MPET-8 Readout system for position sensitive PMTs, coincidence timing" designed by mesytec [30]. One MPET-8 readout system is needed per pair of detector modules to determine the coincidence events detected by the modules. An intelligent central processing device (MCPD2) transmits the data from the MPET- 8 readouts to a PC to be analyzed on their MesyDaq software. Additionally each module is powered by a separate high voltage power supply.

\subsubsection{C-arm for 3D imaging}

A Siemens Arcadis Orbic mobile C-arm capable of cone-beam computed tomography $(\mathrm{CT})$ and 3D image generation is used in this project (see Fig. 1.4). The 
C-arm contains a trolley with dual monitors for imaging display and an imaging module. The imaging module contains an x-ray tube (XRT), which generates x-rays via bremsstrahlung and an image intensifier (II) detector to measure the x-ray signal and produce 2D projection images. The XRT and II face each other on opposite sides of the $\mathrm{C}$ shaped gantry. The patient or object would be placed in the isocenter for $2 \mathrm{D}$ imaging. To acquire 3D images the XRT and II would rotate $190^{\circ}$ around the isocenter collecting $2 \mathrm{D}$ projection images. A reconstruction technique would be used to create a $3 \mathrm{D}$ image from all the acquired 2D projections during the gantry rotation.

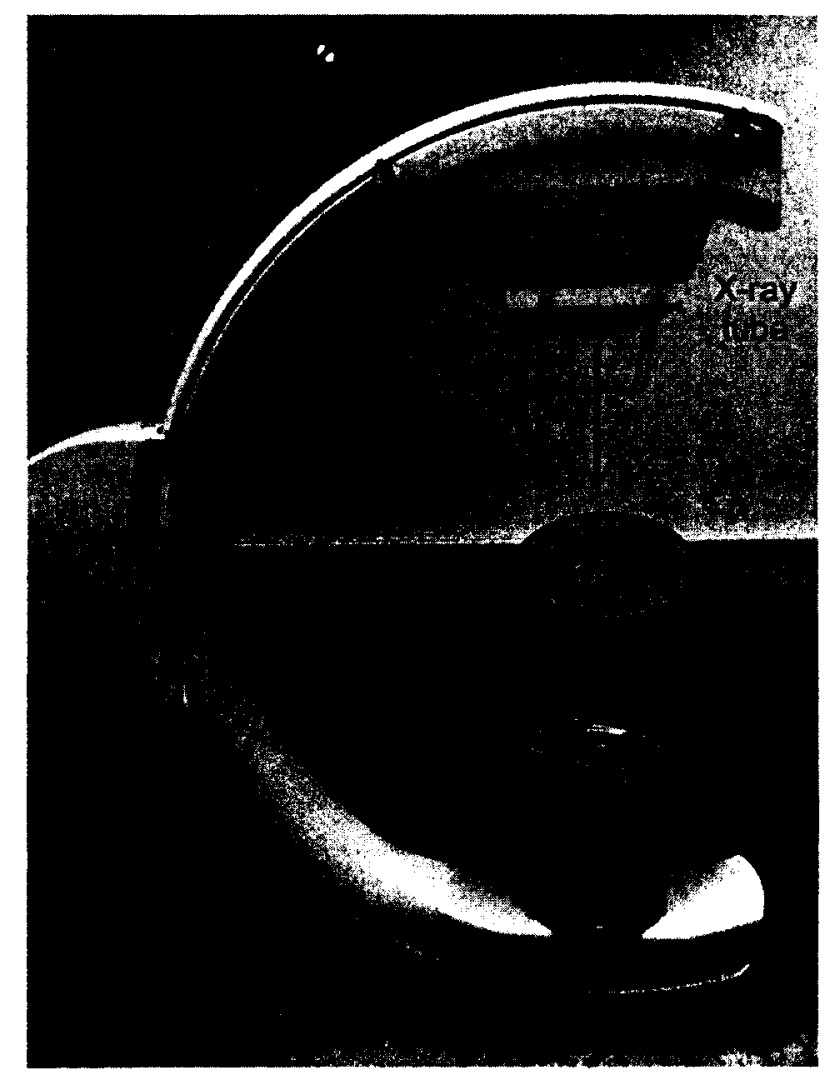

Figure 1.4: Siemens Arcadis Orbic C-arm x-ray system.

Siemens markets this system as the only mobile C-arm they produce capable of $3 \mathrm{D}$ volume reconstruction [31]. The system is designed for intra-operative use in orthopedics and trauma surgery. Physicians are able to image patients during interventional procedures so their progress and results can be immediately checked 
and the physician can react accordingly. The system operates with an II detector designed to considerably reduce $\mathrm{x}$-ray exposure to both patients and staff during use. The C-arm's isocentric design eliminates the need for repositioning of the patient if imaging the patient from more than a single orientation. This system allows for on-site 3D imaging of such things as patient bones, joints, entire spine, and hips.

The image intensifier of this system has a 9 inch $(22.86 \mathrm{~cm})$ field of view, obtains projection images which are $1024 \times 1024$ pixels, with a pixel size of $0.207 \times 0.207 \mathrm{~mm}^{2}$, and can reconstruct a volume of $(12 \mathrm{~cm})^{3}$ at the isocenter.

The Arcadis Orbic C-arm is capable of on-board 3D image reconstruction, however, the complex engineering requirements for a mobile x-ray system capable of gantry rotation inherently result in a significant amount of non-ideal motion of the $\mathrm{x}$-ray scanner and detector from such things as gravity effects during rotation. A good characterization of the $\mathrm{x}$-ray scanner geometry is required for such things as image reconstruction.

\subsubsection{The image intensifier (II) detector}

The image intensifier's purpose is to collect the incident $\mathrm{x}$-ray signal and transform it into an image of the object, however, due to the inherent design of the II the geometrical display of the image is significantly altered thus creating image distortion. These sources of distortion must be corrected for the accurate display of projection images needed in many applications such as 3D image reconstruction (chapter 4) and scanner geometry calibration (described in detail in section 2.1.1).

The II has four primary components housed inside a vacuum tube. The components are: 1) an x-ray absorbing, light emitting input phosphor screen, 2) a photocathode, 3) electrodes positioned to focus the electron beam, and 4) a flat output screen containing a thin anode $[23,32,33]$ (See Fig.1.5). X-rays are emitted from the C-arm $\mathrm{x}$-ray tube and travel through the object before entering the image intensifier 
and striking the input phosphor. The input phosphor is composed of caesium iodide (CsI) in a long thin needle like structure. The CsI needles absorb the $\mathrm{x}$-rays, convert their energy into visible light photons while directing the photons along the length of the needle towards the photocathode [23]. When these photons strike the photocathode they are converted into electrons which are then focused and accelerated towards the output window. The three focusing electrodes (G1, G2, G3) along with the photocathode and the anode of the output window comprise the electronic lens system [23]. Together this system focuses the electrons onto the small output window while maintaining their spatial pattern. Additionally they produce a $25-35 \mathrm{kV}$ electric field which substantially accelerates the electrons towards the output screen producing electronic gain [23]. The output window is constructed with a thin anode $(0.2 \mu \mathrm{m})$, an output phosphor $(4-8 \mu \mathrm{m})$, and a thick output window (14 mm) [23]. The output phosphor converts the incident electrons into visible light which travels through the output window and is digitally collected to produce the image [23]. The anode is a thin coating of aluminum on the top of the output window which simply acts to carry away the charge from the incident electrons.

\subsection{Summary}

This project focuses on the acquisition of accurate 3D images and the co-registration between the PeTrack positron tracking system and the 3D imaging system. Once both of these are achieved the $3 \mathrm{D}$ positions of positron markers tracked by the PeTrack system can be displayed on the $2 \mathrm{D}$ or $3 \mathrm{D}$ image in real-time.

In order to obtain accurate $2 \mathrm{D}$ and $3 \mathrm{D}$ images from an $\mathrm{x}$-ray II, angle dependent distortion must be measured under controlled conditions to allow for the correction of subsequent scans. The process of distortion measurement and correction is explained in chapter 2. 


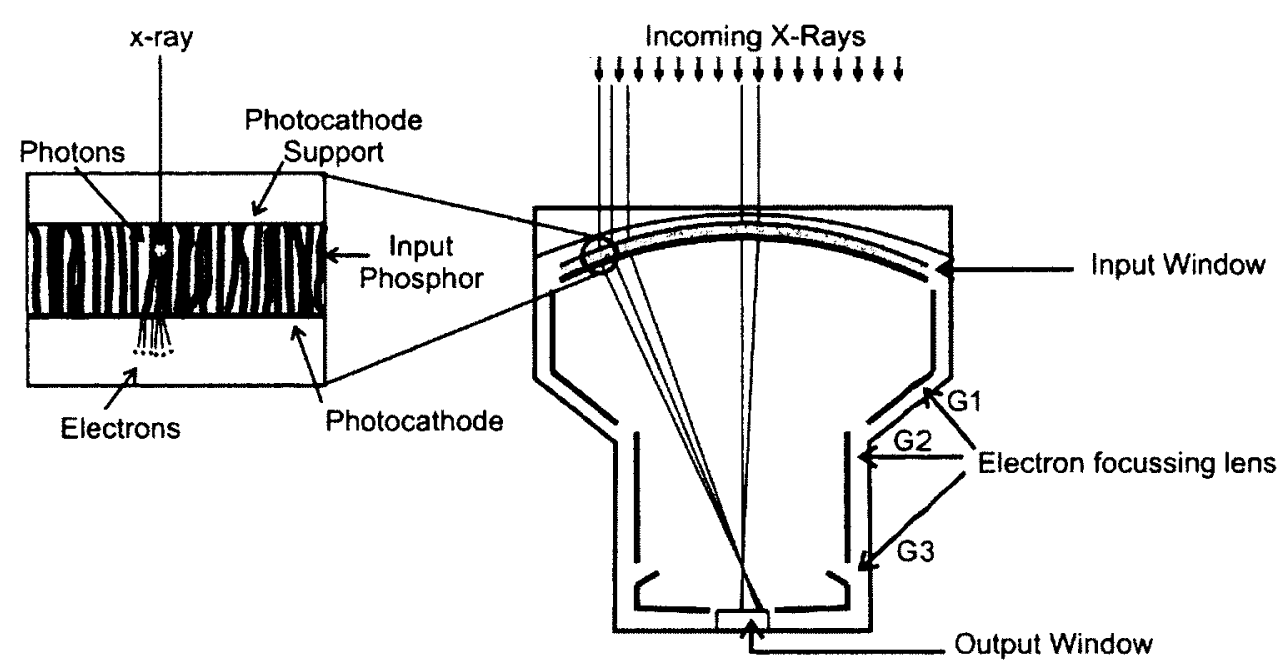

Figure 1.5: Schematic of the image intensifier (II) and II input window.

The method of geometric calibration of the C-arm x-ray scanner is shown in chapter 3. Here the precise behaviour of the scanner is measured for use in both the generation of $3 \mathrm{D}$ images and the co-registration of the $\mathrm{x}$-ray system with the PeTrack system.

Chapter 4 describes the reconstruction procedure to generate $3 \mathrm{D}$ images and further evaluates the performance and ability of the distortion correction and geometric calibration techniques.

The PeTrack detector system had to be geometrically calibrated before accurate localization and tracking could occur. The PeTrack calibration is accomplished with the addition of positron emission markers to the $\mathrm{x}$-ray calibration phantom. This dual calibration creates a common 3D reference frame for the co-registration of the PeTrack system with the $\mathrm{x}$-ray imaging system. The PeTrack calibration and co-registration are presented and discussed in chapter 5 .

Chapter 6 summarizes the results of the project and discusses possible improvements and the future goals of the project. 


\section{Chapter 2}

\section{Distortion: Measurement \& Correction}

\subsection{Overview}

For accurate 3D image generation, image intensifier (II) distortion must be measured so that subsequent projection images can be corrected. Using corrected projection images scanner geometry calibration can be achieved and then a series of corrected $2 \mathrm{D}$ projection images of an object can be reconstructed with the highest accuracy. Due to the fact that II distortion is position and orientation dependent, the distortion measurement \& correction is only valid for scans obtained with the C-arm in the same location, and scans obtained in the same direction (for example the II sweeps in a counter-clockwise "C" instead of clockwise). Hence, the correction can only apply if the distortion is measured when the II transverses the same space with the same orientation. It should also be noted that changes in the local magnetic field can affect the II distortion, so distortion measurements are also dependent on the location of objects in the vicinity of the C-arm II.

The standard approach to correcting inage intensifier (II) distortion always requires the use of a phantom of known specifications. A phantom used for 2D image correction is usually a planar grid phantom that spans the projection image field of view composed of either small radio opaque objects or an opaque material with 
transparent holes [34-37]. The phantom is imaged to generate a distorted projection of the phantom and thus a measurement of distortion at the pre-defined locations of the phantom. Using this measurement and the known specifications of the phantom, some type of interpolating function is used to provide a mapping from any distorted image to its corresponding corrected image for every pixel in the image. This interpolating function is determined for every projection of a scan required for a 3D image reconstruction and the mapping is applied to any subsequent $2 \mathrm{D}$ projections or full scans for $2 \mathrm{D}$ or $3 \mathrm{D}$ distortion corrected image generation.

\subsubsection{Distortion}

The electronic optics used in the II are synonymous in application to those used in standard light optics, therefore the same description of lens aberrations apply to the II which cause image distortion. There are 5 primary Seidel aberrations seen in II's [32]. The following aberrations cause image blurring: spherical aberration, coma, astigmatism, and curvature of field. These blurring affects can be minimized by careful construction of the electronic lens structure. The fifth aberration, distortion by displacement or stretching, is caused by the curvature of the photocathode, termed pin-cushion distortion, and will be further discussed.

\section{Pin-cushion distortion}

The image intensifier (II) optics require the photocathode to be curved however this in turn requires the output screen of the II to have the same curvature. The mapping of the curved input phosphor of the II to the flat output screen, (see Fig. 1.5), creates pin-cushion distortion. This effect is similar to that of a camera lens and will result in greater magnification of the object at distances further from the center of the image as seen in figure 2.1. Pin-cushion distortion can have quite a substantial effect on images generated from an II. Luckily the causes of this distortion are woll 
understood and are only dependent on the specific imaging system. Therefore, it is possible to mathematically describe this distortion by modeling the effect either based on the scanner details or a simple two parameter equation. This view independent distortion correction has been accurately achieved and is described in more detail in section 2.1.3.
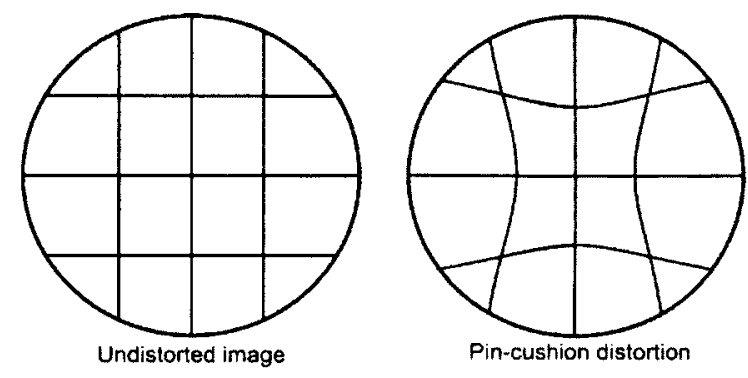

Figure 2.1: An example of the effects of pin-cushion distortion

\section{S-distortion}

S-distortion is another source of II distortion which is not caused by the optics of the II but caused by unavoidable physical affects. S-distortion is due to the earth's and other local magnetic field's influence on the velocity of traveling electrons within the II. S-distortion is therefore dependent not only on local magnetic fields but also on the II's orientation relative to magnetic fields.

As the electrons travel from the photocathode to the output screen the magnetic field acts to both rotate the image and to translate the image. The transverse component of the magnetic field will act on the downward component of electron velocity to translate the image (see Fig. 2.2(a)), whereas the component of the magnetic field which is parallel to the axis of the II will act on the radial component of electron velocity to rotate the image (see Fig 2.2(b)). An example of the effects of S-distortion can be seen in figure 2.3 . 


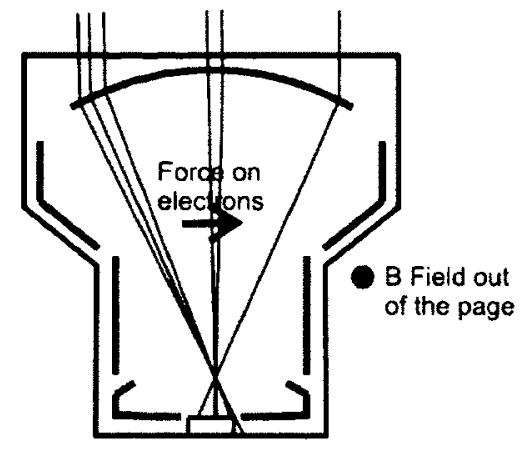

(a) Effects of the transverse component of $B$ field

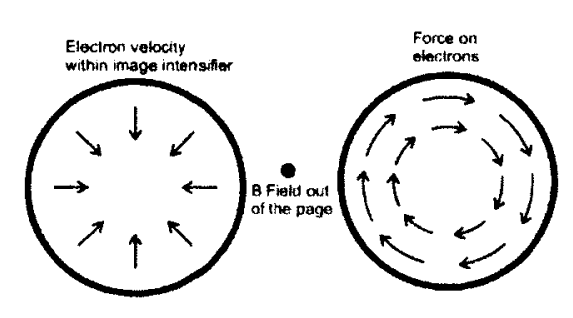

(b) Effects of the parallel component of $B$ field

Figure 2.2: The effects of magnetic fields on electrons traveling within the II.

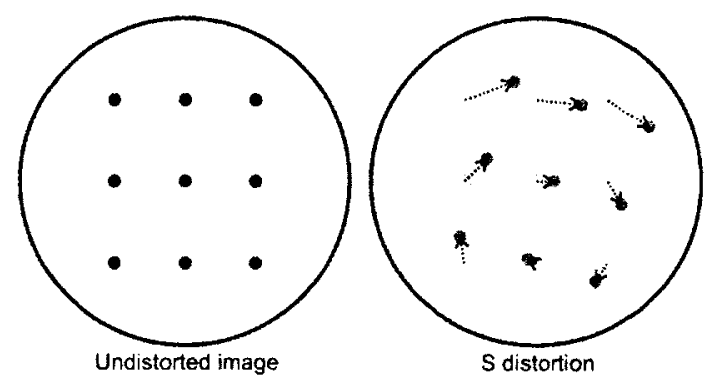

Figure 2.3: An example of the effects of S-distortion

Because the II rotates $190^{\circ}$ during image acquisition these magnetic fields introduce a significant amount of angle dependent S-distortion which is not usually compensated during scanner operation.

\subsubsection{Image intensifier distortion correction in the literature}

Previous authors have used a variety of different interpolating functions to map between the distorted and corrected images. Some authors use higher order bipolynomial fits (a polynomial fitting across both $\mathrm{x}$ and $\mathrm{y}$ directions of the image) to correct image distortion while others allow the phantom to partition the image into smaller 
regions then use a low order bipolynomial to do a regional distortion correction for all regions in the image. Both techniques have been shown in the literature to provide accurate methods of distortion correction.

Fahrig et al. [34] correct image distortion of a $27 \mathrm{~cm}$ diameter x-ray image intensifier (II) using a 5th degree bipolynomial fit. A planar phantom of 336 evenly spaced radio opaque beads is used to measure the distortion of each $2 \mathrm{D}$ projection image obtained at $2^{\circ}$ increments as the II rotates through $200^{\circ}$. The angle dependent correction coefficients are determined by the bipolynomial fitting and the correction coefficients are linearly interpolated at the required angles to correct an image obtained at an arbitrary angle of gantry rotation. These authors further investigate the effect of the order of the polynomial used for correction and determine that from the orders 3 rd to 7 th, a 5 th order bipolynomial is optimal for correction.

In Liu et al. [35], the authors discussed the process of correcting image distortion using a polynomial fit which interpolates for all gantry angles. This method uses a planar phantom composed of a 9 by 9 matrix of copper beads and implements a 5 th degree bipolynomial function to map a single projection image at a specific gantry angle. To extend this to all gantry angles the authors determine the polynomial fits for single projection images at $5^{\circ}$ increments and the 3rd order and higher terms are fitted as a function of gantry angle. This provides a 6 th degree polynomial for image distortion correction as a function of gantry angle.

Schueler et al. [36] describe a fairly simple technique in which a grid phantom is used to partition the image into small triangular regions and a first degree bipolynomial corrects for the local distortion within each region. In their article a grid phantom of lead pellets is used to partition the image into triangular regions. The 3 measured locations of each triangular region give 6 knowns ( $3 x$ 's and $3 y$ 's) to find the 3 parameter polynomial which determines the corrected pixel location in the $\mathrm{x}$ direction and another 3 parameters to determine the corrected pixel location in the 
$\mathrm{y}$ direction for all distorted pixels within that triangular region. Similar polynomials are found for every region in the image and stored to correct distortion of images acquired at that gantry angle. The pixel value in the corrected image is determined using bi-linear interpolation from the four nearest surrounding pixel values from the distorted image.

The authors Wang et al. [37] achieved distortion correction in a similar manner to

Schueler et al. [36] except the image is partitioned by a grid phantom into quadrilateral regions and there is the addition of a correction parameter for the $(x * y)$ cross term. This creates an 8 parameter first degree bipolynomial fitting along the $\mathrm{x}$ and $\mathrm{y}$ directions. The 8 parameter bipolynomial is determined for each four point quadrilateral region in a $2 \mathrm{D}$ image and stored to correct subsequent images. Many images are taken along small intervals of gantry angle from $0^{\circ}$ to $420^{\circ}$, and the correction parameters at many gantry angles are stored as a series of look-up tables. Again to find the corrected pixel value bi-linear interpolation between the four nearest neighbor pixels is done.

\subsubsection{Image intensifier pin-cushion distortion correction from the Siemens on-board system}

The literature demonstrates that II pin-cushion distortion can be easily corrected for.

The works of Pietka \& Huang [33] show that II distortion is simply characterized by: the radius of curvature of the input phosphor screen, the view angle, the distance from the focal spot to the object, and the distance from the focal spot to the input phosphor. The authors derived a mathematical model which can correct the pincushion distortion for any image projection of any II given that the view angle and II design specifications are known.

Additionally, Rudin et al. [32] have shown (see Eqn. 2.1) that II pin-cushion 
distortion can be characterized as a radial distortion from the center of the image and approximated by a simple two parameter ( $\mathrm{E}$ and $\mathrm{D}$ ) odd-power polynomial:

$$
r^{\prime}=r+D r^{3}+E r^{5}
$$

where $r^{\prime}$ is the radial distance of a feature from the center of the II field with distortion and $r$ is the radial distance of the feature without distortion [32]. This corrects for both the geometrically induced effects, from the curvature of the photocathode, as well as any electronic factors due to the electron optics. This technique has the advantages that the shape of the photocathode does not need to be known.

Each image obtained from the Siemens on-board C-arm software has already been corrected for the view independent, pin-cushion distortion but the Siemens system cannot correct for location and orientation dependent S-distortion. In principle, however, S-distortion can be measured and corrected for each II position and orientation.

\subsubsection{Implemented method of distortion correction}

The distortion correction method reported in this study was adapted and modified from [37] with some concepts included from [36]. The regional distortion correction technique seemed to be well suited for the needs of this project as it was an accurate, efficient, and simple method requiring an easy to construct low-cost phantom.

The correction procedure was very similar to [37] using a 4 point quadrilateral mapping. An imaged grid phantom acts to partition the image into many small quadrilateral regions and provided the regions are small compared to the image dis-

tortion an accurate correction can be achieved. Unlike [37], however, there were some special considerations incorporated for regions at the edge of the image field of view (FOV). Further, parameters were measured at every interval of gantry rotation so 
no interpolation between correction parameters was needed for arbitrary imaging angles. Section 2.2 gives an in-depth description of the method of distortion correction implemented in this study.

\subsection{Distortion measurement and correction proce- dure}

This method implemented a standard planar grid phantom of radio opaque steel balls to measure II distortion at each angular projection view. Distortion was measured and correction parameters were stored for all 100 projections of a full $190^{\circ} \mathrm{C}$-arm scan. The correction procedure can then be used to correct angular dependent II distortion for any subsequent scans acquired under the same conditions.

The stored correction parameters are used in the correction procedure to "pull" the distorted pixel to the correct pixel location in the undistorted image. The correction parameters therefore, are determined to find the distorted image location for any given undistorted pixel.

\subsubsection{Planar grid phantom}

A planar grid phantom was constructed to measure distortion, see Fig. 2.4. The grid was constructed by drilling holes in a $12 \times 12 \times 1 / 2$ inch square piece of Lucite using a computer automated system. The holes were arranged in a $17 \times 17$ matrix centered at the center of the Lucite and contained 289 radio opaque steel balls of $3.5 \mathrm{~mm}$ diameter. The center to center distance between the balls in the matrix was $14.0 \mathrm{~mm}$. The balls of the grid phantom completely span the image field of view. The specifications of the phantom were designed based on cost and an estimated acceptable distortion correction accuracy. 


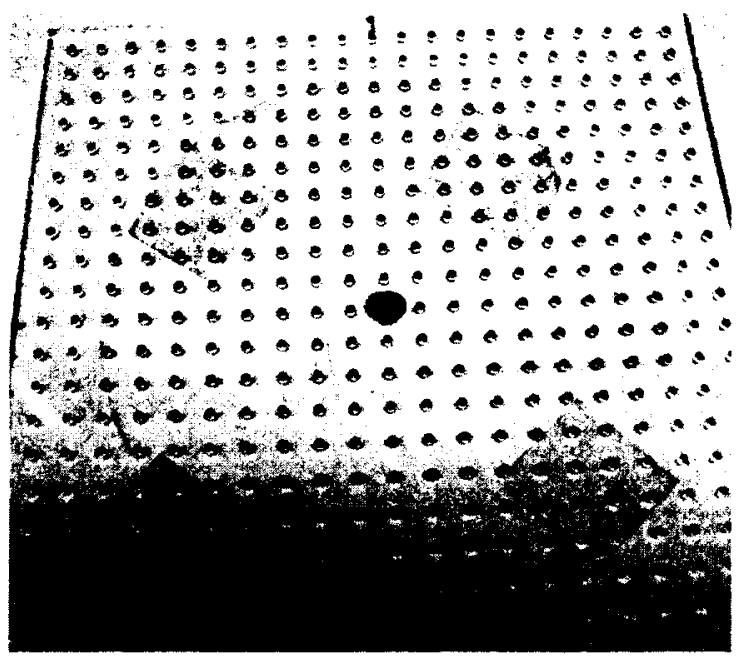

Figure 2.4: Planar grid phantom used for distortion correction.

\subsubsection{Distortion measurement}

The planar grid phantom is attached directly to the face of the image intensifier and imaged to provide a measurement of distortion, see Fig. 2.5. The planar grid phantom acts to partition the the image into many small quadrilateral regions. Each region is established by four measured points in the image.

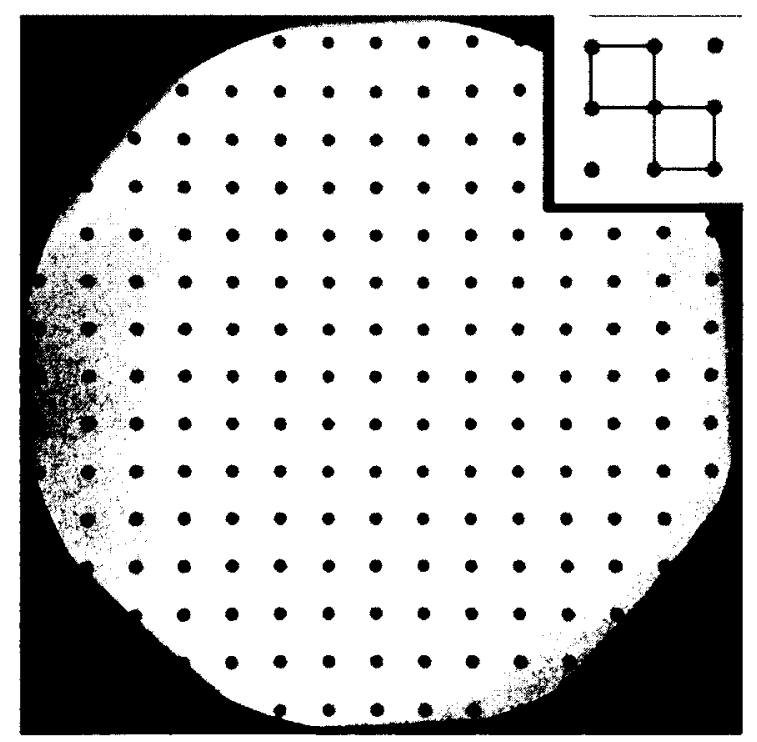

Figure 2.5: Grid phantom imaged directly on the II. The inset visually displays the quadrilateral regions partitioned by the phantom. 
The phantom was imaged during a full scan of the $\mathrm{C}$-arm acquiring 100 projection images around $190^{\circ}$ of gantry rotation. These 100 projection images indicate the locations of every ball in the image field of view (FOV) and thus the distortion measurement of every projection. The images were processed automatically using an ImageJ macro [38]. The images were thresholded at a predefined level to separate the radio opaque balls from the background and then a function was used to identify the position of every ball in the image. The sub-pixel ball locations were determined by a center of mass weighting from the thresholded (binary) image. For subsequent use, each ball was assigned an $(i, j)$ index based on its location relative to the center of the image (such that the central ball of the image has index $(0,0)$ ). The index and location of every ball from every projection was stored.

\subsubsection{Ideal undistorted estimation}

At this stage an approximation was made to determine an ideal undistorted measurement of the ball locations of the imaged phantom. Under ideal conditions, hence no distortion or scanner geometry affects, each projection of the grid should be a rigid matrix of evenly spaced balls. Furthermore, under ideal conditions the 100 projection images of the grid as the $\mathrm{C}$-arm rotates $190^{\circ}$ should not rotate or translate from one projection to the next. Therefore an ideal undistorted measurement should simply be only one ideal rigid grid of ball locations. The assumption that the center of a single projection image has the least amount of distortion is valid for both pin-cushion and S-distortion.

Projection frame \#50 was selected arbitrarily and the locations of the center 3 by 3 balls in the distorted image are assumed to be ideal. These central $9(x, y)$ locations provided 18 scalars to a $\chi^{2}$ minimization method to fit for 5 parameters $\left(x_{0}, y_{0}\right), \Delta x, \Delta y$, and $\theta$. These 5 parameters link the predetermined $(i, j)$ indices to ideal $(x, y)$ locations on the grid. 


$$
\left[\begin{array}{l}
x \\
y
\end{array}\right]=\left[\begin{array}{cc}
\cos \theta & -\sin \theta \\
\sin \theta & \cos \theta
\end{array}\right]\left[\begin{array}{l}
i \Delta x \\
j \Delta y
\end{array}\right]+\left[\begin{array}{l}
x_{0} \\
y_{0}
\end{array}\right]
$$

The parameters determined allow for a fixed shift $\left(x_{0}, y_{0}\right)$, fixed rotation $\theta$, and independent pitches $\Delta x$ and $\Delta y$. The ideal location of every ball in all 100 projection images was then found using these 5 parameters and its associated index.

\subsubsection{Regional distortion correction: parametrization}

The ball locations of the distorted images have been measured and their ideal undistorted location can be calculated with the fitted 5 parameters, Eqn. 2.2. Every image has been partitioned into many small quadrilateral regions established by four points. Each distorted quadrilateral region corresponds to a calculated ideal square region. For every quadrilateral region an 8 parameter, $a-h$, bipolynomial function was used to map the corners of the distorted quadrilateral, $(\hat{x}, \hat{y})$, to the corners of the ideal square, $(x, y)[37]$.

$$
\widehat{x}=a x+b y+c x y+d \quad \widehat{y}=e x+f y+g x y+h
$$

Equation 2.2 gives the distorted image location, $(\hat{x}, \hat{y})$, in terms of the correction parameters and an ideal pixel location, $(x, y)$. This form is a repercussion of the correction procedure in which the corrected image pixels are filled by pulling the correct pixel from the distorted image, hence the need to determine the distorted image locations.

Using the matrix below provided a mapping for every point in the ideal square region to a corresponding point inside the quadrilateral region (see Fig. 2.6). 


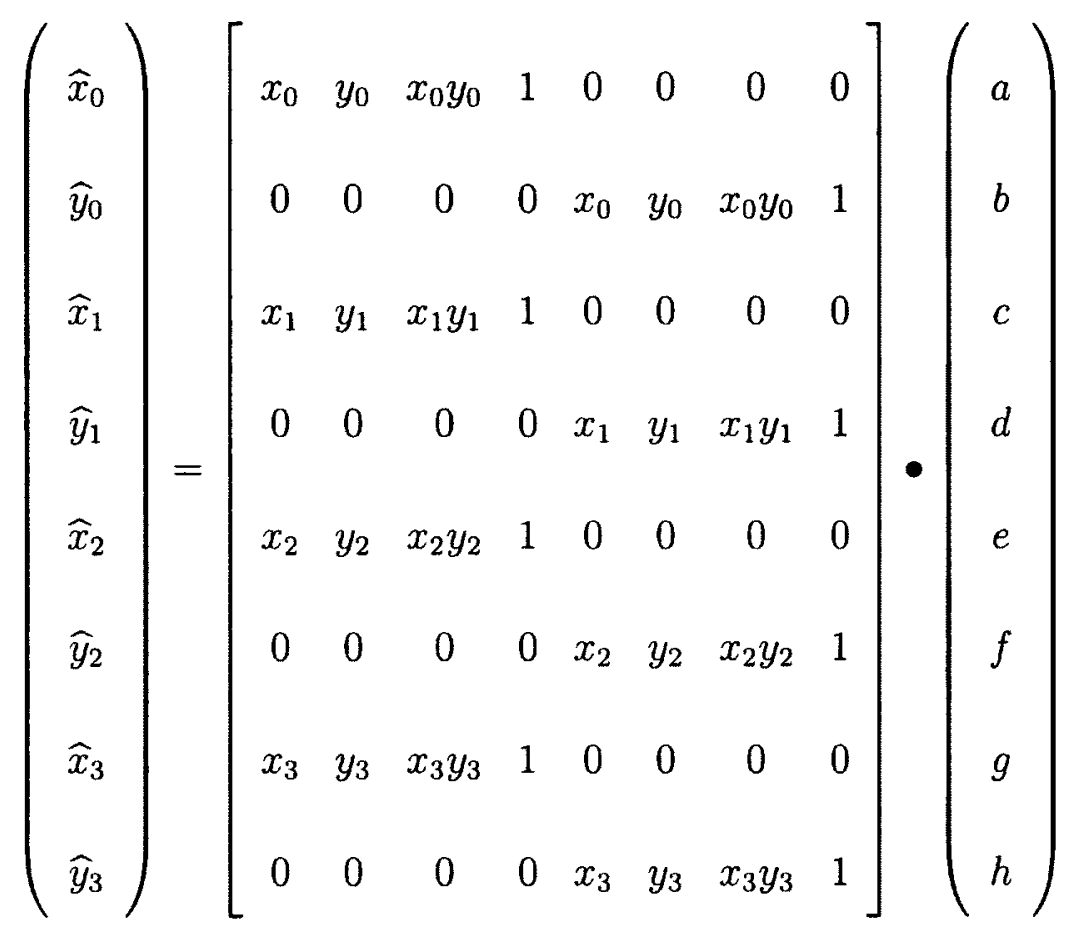

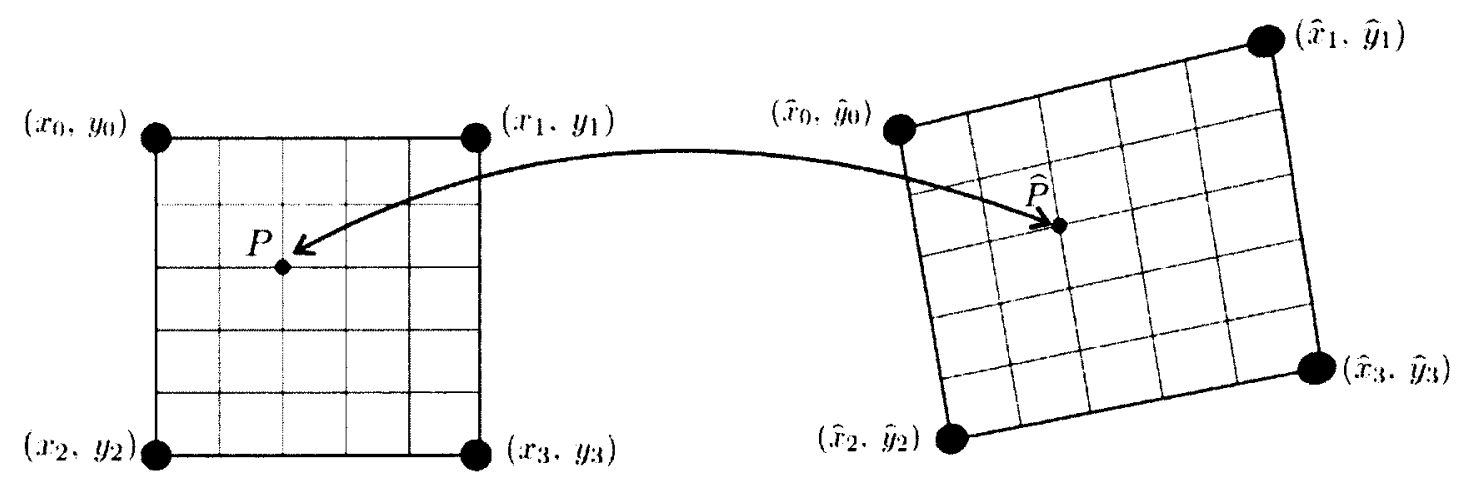

Figure 2.6: Mapping of each point from the ideal image to the distorted image.

This parametrization was repeated for every quadrilateral region in the distorted projection image giving a separate 8 parameter bipolynomial to correct for each region. Following this procedure, all other distorted projection images were mapped back to the single ideal undistorted grid using their own set of bipolynomials for each region.

The polynomials found for every region contained in all 100 projection images 
were stored to be used to correct the distortion in subsequent scans.

\subsubsection{Regions at the edge of the field of view}

The mapping procedure was modified near the edge of the image field of view (FOV) where one or more balls defining a quadrilateral region are missing, as seen in Fig. 2.7 .

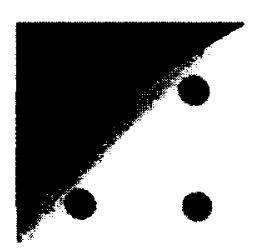

Figure 2.7: An example of triangular regions in which one or more balls are outside of the field of view.

If one ball is missing, a lower order polynomial fit is used to map between triangular regions [36]. In this case the $x * y$ cross term is omitted and the 3 points of the triangular region are used to fit a 6 parameter bipolynomial (see Eqn. 2.5). This mapping is also applied to the adjacent triangular region with only two points in the image FOV to obtain correction parameters for the full quadrilateral region.

$$
\widehat{x}=a x+b y+d \quad \widehat{y}=e x+f y+h
$$

If two or more balls were missing the polynomial from the closest adjacent quadrilateral region was extended to the truncated quadrilateral, see Fig. 2.8.

Again the obtained correction parameters are stored to be used to correct subsequent scans.

This correction process undoubtedly decreases the accuracy of distortion correction at the edge of the image field of view however this decrease in fidelity is assumed to be acceptable given our purposes. 


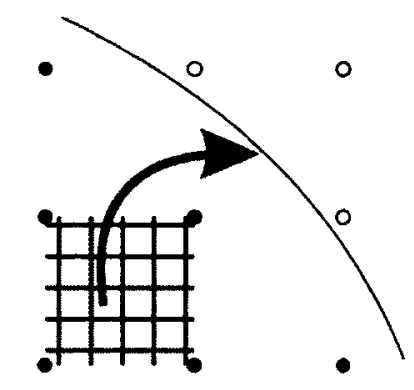

(a) regions with only one ball
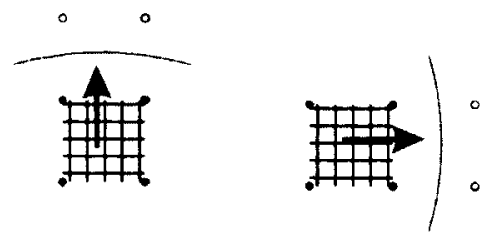

(b) regions with two balls

Figure 2.8: Parameterization of regions with two or less balls in the image FOV.

\subsubsection{Image correction}

The correction parameters have been obtained and stored for every region of every projection so that any image acquired under the same conditions can be corrected by pulling the correct distorted pixel value into the corrected image. The corrected image is generated by determining the value of each pixel one by one.

To determine the corrected image pixel value, first the corrected pixels corresponding location in the distorted image is determined within sub-pixel accuracy. This is done using the 8 correction parameters determined for each region in the image, however, the appropriate region must first be determined. This is done by applying the inverse of Eqn. 2.2 to the corrected pixel location, $(x, y)$, to determine an associated $(i, j)$ index and hence the associated region.

Once the location in the distorted image is determined to within sub-pixel accuracy then the four pixels nearest to that location are used in a gray level bilinear interpolation to calculate the pixel value in the corrected image (see Eqn. 2.6 and Fig. 2.9). In Fig. 2.9 and Eqn. 2.6 the variables $Q_{i, j}$ are the four nearest pixel 
values in the distorted image, and the value $Q_{c}$ is the corrected pixel value, and the sub-pixel point $\mathrm{P}$ is at $(\hat{x}, \hat{y})$

$$
Q_{c}=\left(y_{2}-\hat{y}\right)\left[\left(x_{2}-\hat{x}\right) Q_{11}+\left(\hat{x}-x_{1}\right) Q_{21}\right]+\left(\hat{y}-y_{1}\right)\left[\left(x_{2}-\hat{x}\right) Q_{12}+\left(\hat{x}-x_{1}\right) Q_{22}\right]
$$

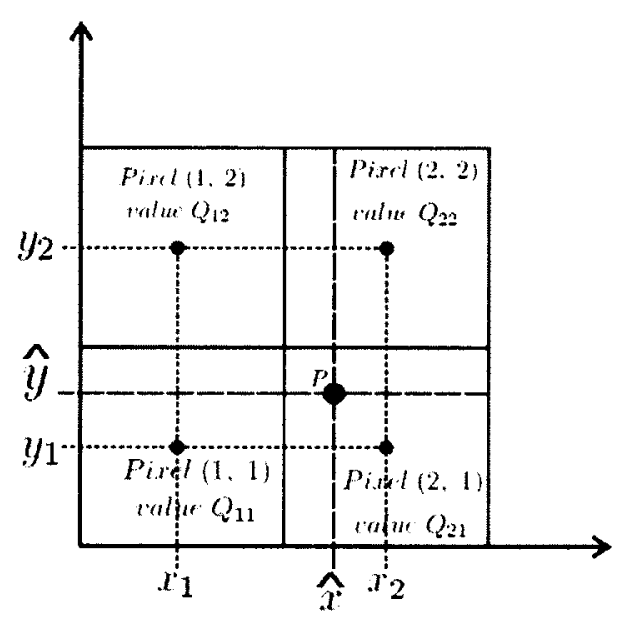

Figure 2.9: Illustrative example of the process of gray level bilinear interpolation.

This procedure is repeated to find the pixel value of each pixel of all 100 corrected projections of the scan.

\subsubsection{Distortion measurement \& correction procedure overview}

The process of measuring distortion requires ImageJ [38] for image pre-processing, a non-linear $\chi^{2}$ fitting program to define the ideal/undistorted grid, and a Matlab program to find the polynomial coefficients used for mapping. This process required approximately 5 minutes of computer processing time and about 10 minutes of operator time for all 100 projection images. The distortion correction procedure was 
managed automatically using a Matlab program, taking approximately 25 seconds of computation time per projection, or about 42 minutes per scan.

\subsection{Distortion correction evaluation}

The distortion correction procedure was done for a full 100 projection scan. All subsequent scans to be corrected were imaged with the $\mathrm{C}$-arm in the same location and with the same scan direction (ie. scans starting with the II closest to the C-arm support structure and ending with the XRT closest to the support structure).

A single projection was selected and corrected to evaluate the distortion correction procedure of a single image. The degree of distortion was also inspected for a full 100 projection scan.

\subsubsection{Evaluation for a single projection image of the grid}

The distortion correction was evaluated for a single projection by imaging the gird phantom on the II in a different location than the correction grid. A rectangular lattice was fitted to all the ball locations in both the corrected and distorted images. In the distorted image the distortion will not allow the ball locations to construct a rigid evenly spaced lattice. The residual distance between the lattice and the distorted ball locations will give an indication of the severity of the distortion (see Fig. 2.10(a)). Alternatively, if the distortion correction is flawless the ball locations in the corrected image will form a perfect lattice. The residual error between the fitted lattice and the actual ball locations will represent the performance of the distortion correction (see Fig. 2.10(b)).

The calculated RMS error between the corrected grid and the fitted lattice was 0.63 pixels (with maximum grid point difference of 2.01 pixels); and the RMS error 


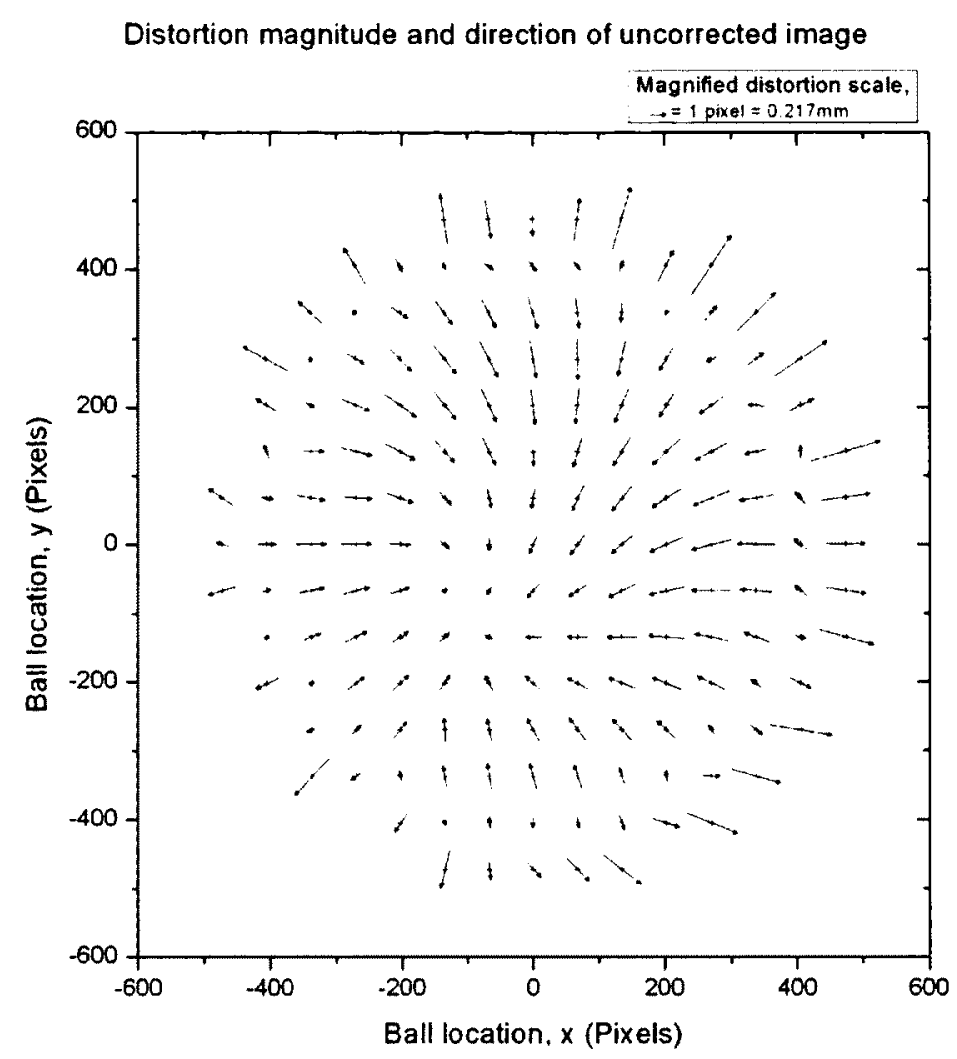

(a)

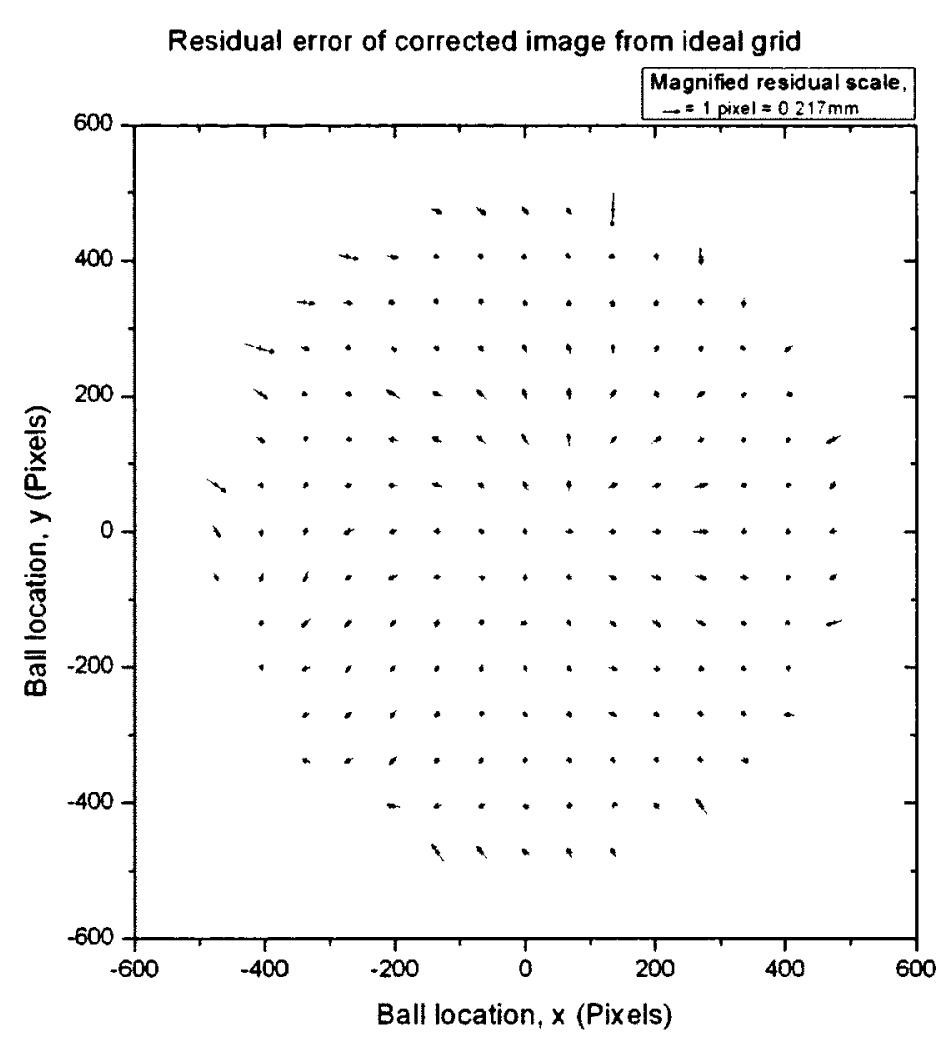

(b)

Figure 2.10: Illustration of uncorrected distortion (a), and an indication of the fidelity of the distortion correction (b). Distortion/residual error is represented by an arrow depicting the magnitude and direction at the $(x, y)$ pixel location in the image shown by a cross at the center of the arrow.

between the distorted (uncorrected) grid and its fitted lattice was 1.71 pixels (maximum difference of 3.7 pixels). The maximum grid point difference is shown to be a locations on the edge of the field of view. This is expected as the distortion correction method degrades when vertices of a quadrilateral region are outside the field of view.

\subsubsection{Degree of distortion during a full scan}

To inspect the degree of distortion incurred during a $190^{\circ}$ scan 100 projection images of the grid phantom before correction were observed and the distorted grid points 
of each projection were plotted as red circles superimposed on one of the corrected projection images (see Fig. 2.11). This image provides a visual description of the magnitude of the corrections.

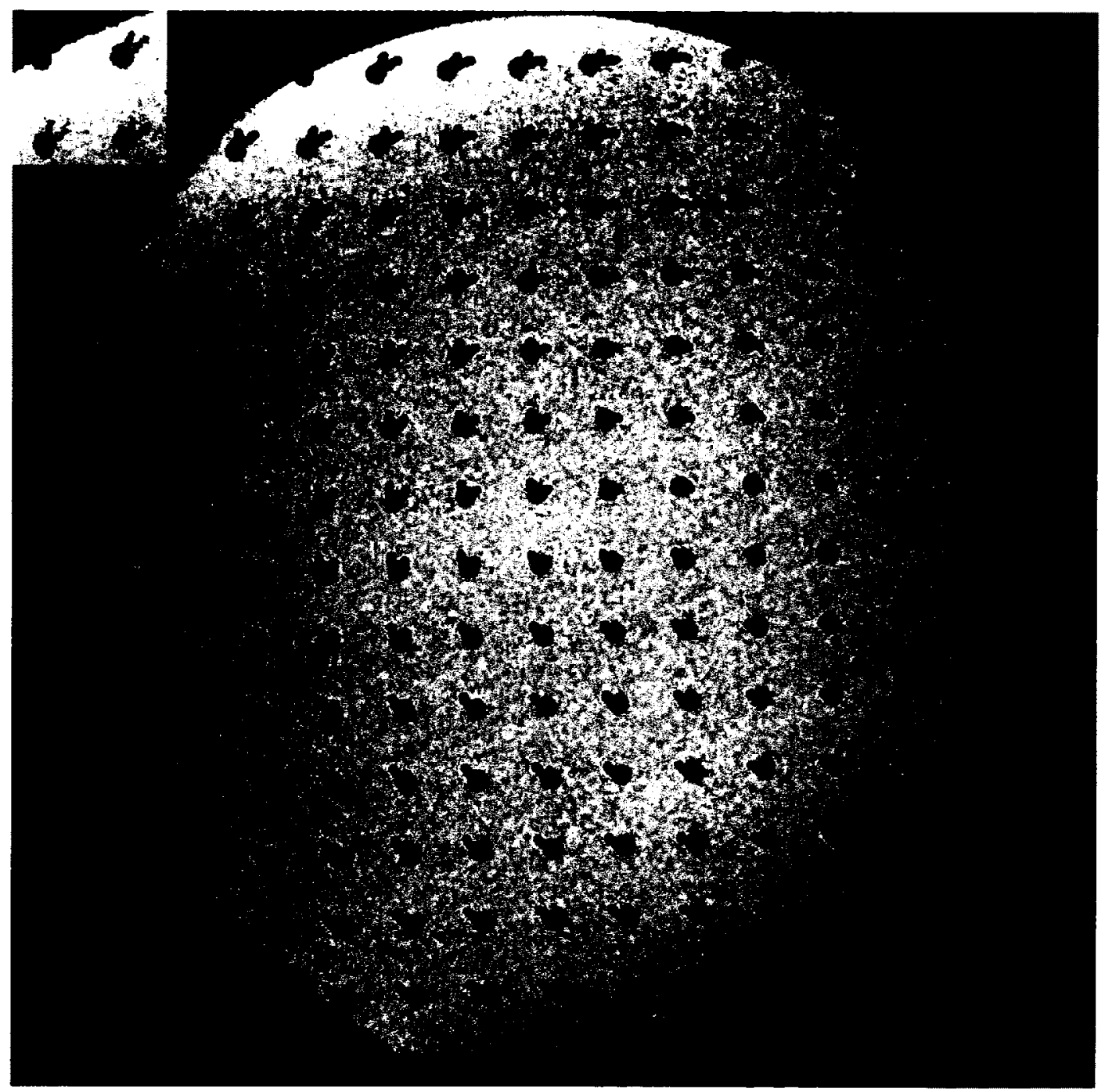

Figure 2.11: Distorted ball locations plotted on corrected projection image \#50 to illustrate the magnitude of the distortion. The inset shows a magnified view of the top left corner. 


\subsection{Discussion}

Our distortion correction evaluation yielded a mean error of 0.63 pixels with a maximum error of 2.01 pixels. Fig. 2.10 above shows that the error around the edge of the image FOV is substantially larger than in the rest of the image and that the maximum error is somewhat of an aberration. The error at the edge of the image field of view is substantially higher as this corresponds to quadrilateral regions in which one or more of the vertices was outside of the image FOV and the correction parameters for that region had to be determined by a less accurate means.

In comparison to the similar methods of Wang et al. [37], which had a correction error of 0.05-0.12 pixels, and Scheuler et al. [36], which had a mean error of 0.10 pixels and a maximum error of 0.36 pixels, our correction error is fairly large. This large discrepancy of the errors can be attributed to one main factor, the grid phantom specifications. The accuracy of the distortion correction is directly related to the size of the correction regions, which in our case were significantly larger with a center to center distance of $14 \mathrm{~mm}$. In contrast, Wang et al. corrected quadrilateral regions composed of markers separated by $5.08 \mathrm{~mm}$ center to center distance. This phantom had regions almost one 10 th of the size of ours. If our phantom were constructed with a much smaller center to center distance between balls and a smaller ball diameter and smaller ball spacing leading to more correction regions in the image field of view the correction accuracy would undoubtedly improve. Further, a smaller quadrilateral region size would allow more full quadrilateral regions to be discerned at the edge of the field of view. Ultimately the correction accuracy desired for PeTrack localization and tracking on images was attained.

The effects of S-distortion during a full $190^{\circ}$ scan are shown to be quite significant, and the necessity for II distortion correction is justified. The correction parameters 
are stored to correct distortion for any subsequent scans done under the same conditions. The distortion correction will be further evaluated by observing its effects on the scanner geometry calibration (chapter 3) and the visual effects seen on 3D reconstructed images (chapter 4 ). 


\section{Chapter 3}

\section{Scanner Geometry Calibration}

\subsection{Overview}

In order to achieve 3D image reconstruction from an $\mathrm{x}$-ray scanner geometric calibration is needed. Geometric calibration is the process of determining the exact detector and source locations and orientations during the scan. Algorithms used for image reconstruction assume that the $\mathrm{x}$-ray source and the detector follow a perfectly circular trajectory [39]; if there are nonidealities in this trajectory high-quality 3D images cannot be constructed. During C-arm rotation there is a significant amount of non-ideal motion such as bearing irregularities, sagging, bending, and stretching due to gravity [39] which must be measured.

Geometric calibration measurements can be done in two main ways: by monitoring the motion of external markers placed on the C-arm, or by using an image based marker system to monitor gantry motion during rotation. The latter technique is implemented more often in the literature and proves to have higher fidelity. Some image based calibration objects have been used during patient scans to calibrate scanner geometry individually during each scan [40]. These calibration objects however cannot be placed in the center or span the image field of view. Other techniques characterize gantry motion before image acquisition $[39,40]$. These techniques prove to more 
accurately characterize the scanner geometry however require that the motion of the gantry is reproducible at some level (from days to weeks or even months) $[39,40]$. If the gantry motion is characterized before image acquisition any acquired image data sets can be digitally processed to corrected for nonidealities before reconstruction or knowledge of the motion could be simply incorporated into the back projection algorithm used for reconstruction [39].

With careful selection of an appropriate calibration object and a robust geometric calibration method, the full transformation matrices (projection, rotation, and translation) can be determined relating a 3D point in space precisely to the projections of a scan. A cone beam CT imaging system requires nine parameters to completely specify the geometry calibration: three for the source position, three for the detector position, and three for the detector orientation [1].

In the case of imaging with an image intensifier (II), the effects of distortion are inter-tangled with those of calibration. Therefore, if distortion correction is accomplished before geometric calibration the distortion correction method will correct for some of the geometry effects. On the other hand, if no distortion correction is completed the x-ray scanner geometric calibration will correct for some of the effects of distortion.

\subsubsection{Siemens geometric calibration method}

The Siemens on-board C-arm system is capable of 3D image reconstruction so geometric calibration is performed. Siemens does their own method of C-arm geometric calibration described in [40]. They use a helical phantom design with radio opaque beads of different sizes placed in a thin cylinder of Lucite (see Fig. 3.1). The size and the arrangement of the beads allow for automatic detection and labeling of the beads to facilitate the calibration procedure. The phantom is aligned in the iso-center and 


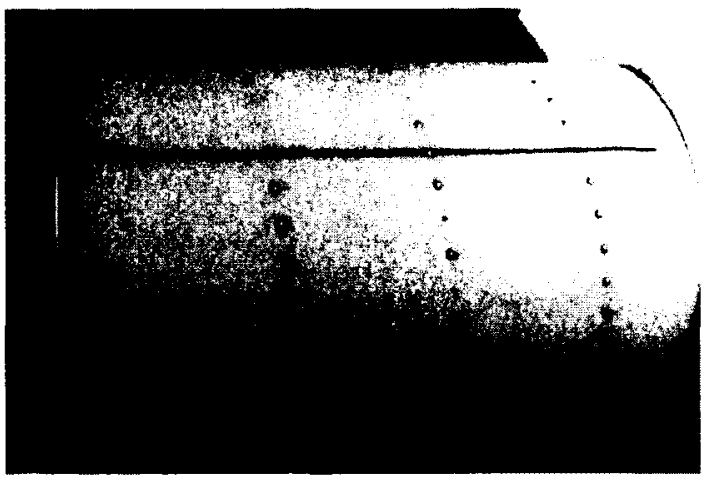

(a) Picture of the calibration phantom

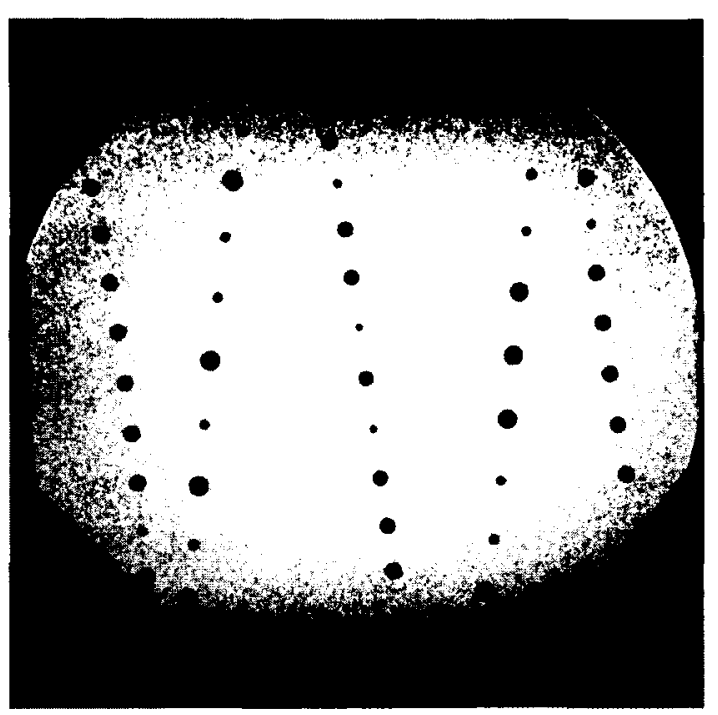

(b) Projection image of the calibration phantom

Figure 3.1: Siemens geometric calibration phantom.

scanned with the C-arm system in calibration mode. It should be noted that this calibration cannot correct for the inherent S-distortion seen in every projection image, it can only estimate the geometry of the scanner.

\subsubsection{Purpose for off-line geometric calibration}

There are two purposes in which off-line geometric calibration must be performed in this study. The first purpose is to achieve distortion-corrected high-quality $3 \mathrm{D}$ image reconstruction. This process can only be done off-line in order to correct distortion in the 100 projection scan of the object before reconstruction and also to correct the 100 projection scan of the calibration object used to preform geometric calibration of the scanner; a step which is required before image reconstruction.

The second purpose is to provide a common 3D reference frame for both the C-arm imaging system and the PeTrack 3D localization system in order to co-register the two systems. This is only possible with a dual calibration phantom. As a result, the 
Siemens method of C-arm geometric calibration cannot be used and a new method must be implemented which uses a different phantom. The dual calibration phantom will be described in more detail in chapter 5 .

\subsection{Geometric calibration method}

The geometric calibration process implemented in this study was done in collaboration with Dr. Rolf Clackdoyle and Dr. Catherine Mennessier from Laboratoire Hubert Curien and CNRS. The calibration method implemented their recently published calibration procedure [1] to completely specify the geometry of our C-arm scanner. There were some modifications made to the calibration phantom design.

The geometric calibration method uses an image based marker system to monitor the motion of the $\mathrm{C}$-arm gantry during rotation. The calibration follows the classical approach of imaging a phantom to map known points of the phantom to their projection. The fully analytic method determines all 9 geometric parameters to specify the geometry of our C-arm scanner: the focal length and the projection of the source on the detector i.e. the detector position ( 3 scalars), the detector orientation (the 3 Euler angles), and the source position ( 3 scalars), see Fig. 3.2. The 9 parameters are found separately for each projection image and each image is calibrated independently. No assumptions need to be made about the source trajectory or the detector position of the C-arm. The calibration object is imaged prior to patient image acquisition and the calibration parameters are incorporated into the reconstruction algorithm to subsequently provide high quality image reconstruction.

A unique $\mathrm{x}$-ray geometric calibration phantom was constructed to implement the calibration method on our x-ray scanner. The mathematical model derivation to determine the analytic formula is notably not very complex. Further, the method can be applied to any scanning geometry as well as other imaging modalities such as 


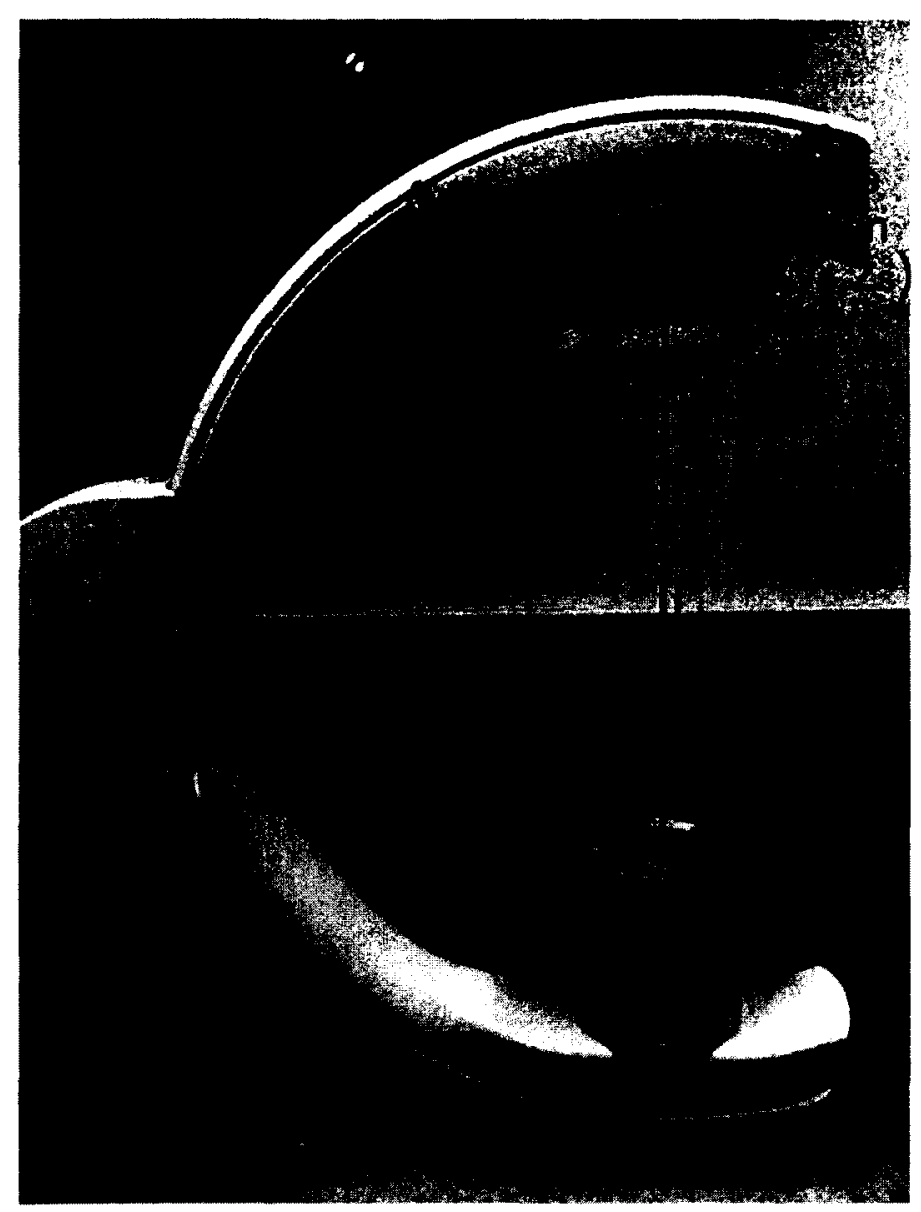

Figure 3.2: Geometric calibration parameters labeled on $\mathrm{C}$-arm.

single photon emission computed tomography (SPECT). This study marks the first time this calibration method has been used and validated with real data.

\subsubsection{Geometric calibration phantom}

The design of the phantom was unique and specific to our $\mathrm{x}$-ray scanner. The phantom was composed of 6 steel balls $(2.5 \mathrm{~mm}$ in diameter) extended from the center of a cube by aluminum tubes (see Fig. 3.3). It was necessary to position the phantom in the $\mathrm{C}$-arm carefully such that during a scan the ball projections did not overlap thus creating a loss of information. As a result, to help with alignment a thin copper wire was attached around the center of the cube, such that when the phantom was aligned 
properly the wire would appear as a line instead of an ellipse in a projection image. Also, to facilitate imaging, a phantom holder case was constructed. The cube and the aluminum tubes used to construct the phantom had to be very precisely machined in order to ensure an accurate calibration. The phantom was constructed with a desired precision of $\pm 50 \mu \mathrm{m}$. for each phantom ball location.

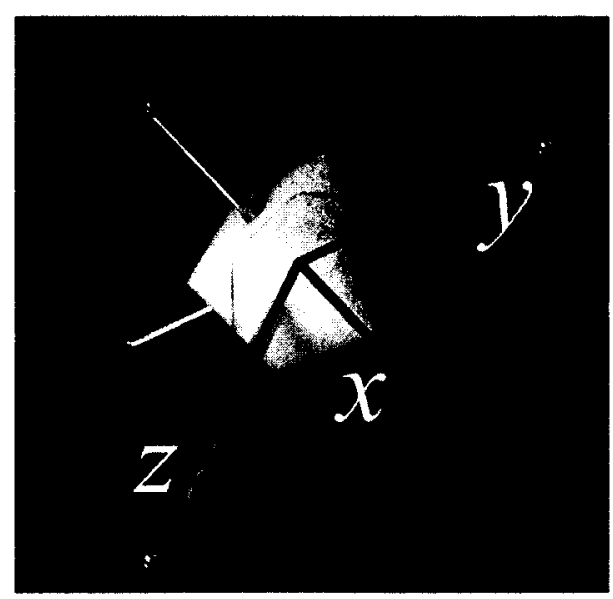

Figure 3.3: X-ray geometric calibration phantom.

This phantom design differed from that proposed in [1]. The original method suggested a phantom in which the steel balls are suspended from the cube at distance of $k$, and that all the balls are suspended at the same length, ie. $k=k_{x}=k_{y}=k_{z}$. This was designed to ensure the projections of the balls do not overlap during the scan [1]. This unfortunately was not the case for our C-arm scanner because the source to detector distance was too large, so the phantom design was modified with $k: k_{x}=30 \mathrm{~mm} k_{y}=40 \mathrm{~mm} k_{z}=50 \mathrm{~mm}$, and minor adjustments were made to the calibration method.

\subsubsection{Mathematical formalism of geometric calibration}

A brief description of the mathematical formalism, which describes how the geometric calibration parameters are determined, follows, though a more in depth description 
can be found in Mennessier et al. [1]. The method determined the 9 parameters using the known specifications of the phantom and images of the 6 ball phantom which gave 12 knowns; a $(u, v)$ position for each ball in the projection image.

The formalism starts by identifying the mathematical description of how a point in $3 \mathrm{D}$ space would be projected onto the detector or image in terms of the parameters under investigation. The calibration parameters are then expressed in terms of three $3 \mathrm{D}$ vectors. Taking advantage of the known phantom locations the 3 vectors are expressed in terms of the of the physically known ball locations relative to the center of the phantom and the 12 known phantom projection locations $(u, v)$. Thus the solution of the vectors is an overdetermined system. The 3 vectors can then describe 15 calibration parameters, 9 of which specify the detector orientation but are later needed for reconstruction purposes. The following describes this process with all the relevant equations.

A projection point $(u(\vec{r}), v(\vec{r}))$ specifies where a point, $\vec{r}$, in 3D space would be projected onto the detector, see Fig. 3.4. Equation 3.1 describes the projection location of point $\vec{r}$, given: the projection point of the source onto the detector, $\left(u_{s}, v_{s}\right)$, the distance from the source to the detector, or focal length $f$, the vector describing the location of the source, $\vec{s}$, and the 3 detector unit vectors, $\hat{u}, \hat{v}, \hat{n}$, (which describe the detector orientation).

$$
u(r)=u_{s}+f \frac{(\vec{r}-\vec{s}) \cdot \hat{u}}{(\vec{r}-\vec{s}) \cdot \hat{n}} \quad v(r)=v_{s}+f \frac{(\vec{r}-\vec{s}) \cdot \hat{v}}{(\vec{r}-\vec{s}) \cdot \hat{n}}
$$

A projection image of the phantom gives 6 projection locations, $\left(u_{i}, v_{i}\right)$, for the 6 phantom points $\overrightarrow{r_{i}}$ for $i=1 \rightarrow 6$. The phantom points are known relative to the center of the phantom:

$$
\vec{r}_{1}=\left(k_{x}, 0,0\right) \quad \vec{r}_{2}=\left(-k_{x}, 0,0\right) \quad \vec{r}_{3}=\left(0, k_{y}, 0\right)
$$




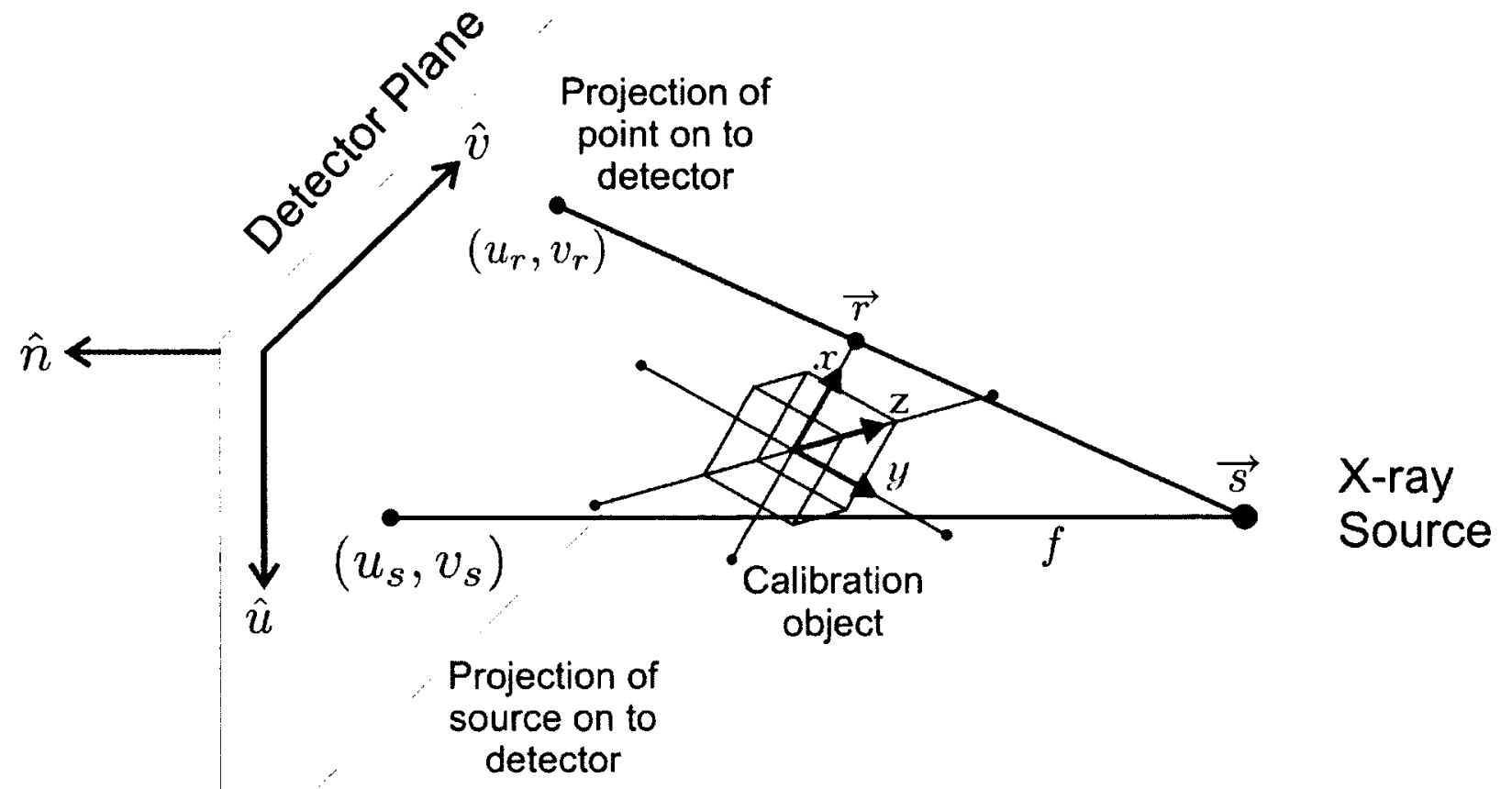

Figure 3.4: Projection of a point in space onto the detector with relevant geometric parameters indicated.

$$
\vec{r}_{4}=\left(0,-k_{y}, 0\right) \quad \vec{r}_{5}=\left(0,0, k_{z}\right) \quad \vec{r}_{6}=\left(0,0,-k_{z}\right)
$$

Nine intermediate parameters are introduced in the form of 3 vectors, $\vec{a}, \vec{b}, \vec{c}$, to describe the 9 sought after geometric calibration parameters. The solution to the vectors, $\vec{a}, \vec{b}, \vec{c}$, can be determined from the imaged locations of the 6 marker locations and the known values of $k_{x}, k_{y}$, and $k_{z}$. The components $a_{x}, b_{x}$, and $c_{x}$ are determined using the imaged locations $\left(u_{1}, v_{1}\right)$ and $\left(u_{1}, v_{2}\right)$ and the value $k_{x}$, see Eqn. 3.2. The other components are found similarly.

$$
a_{x}=\frac{2 u_{1} u_{2}\left(u_{2}-u_{1}\right)+\left(u_{1} v_{2}+u_{2} v_{1}\right)\left(v_{2}-v_{1}\right)}{k\left(\left(u_{2}-u_{1}\right)^{2}+\left(v_{2}-v_{1}\right)^{2}\right)}
$$




$$
\begin{aligned}
& b_{x}=\frac{2 v_{1} v_{2}\left(v_{2}-v_{1}\right)+\left(u_{1} v_{2}+u_{2} v_{1}\right)\left(u_{2}-u_{1}\right)}{k\left(\left(u_{2}-u_{1}\right)^{2}+\left(v_{2}-v_{1}\right)^{2}\right)} \\
& c_{x}=\frac{\left(u_{2}+u_{1}\right)\left(u_{2}-u_{1}\right)+\left(v_{2}+v_{1}\right)\left(v_{2}-v_{1}\right)}{k\left(\left(u_{2}-u_{1}\right)^{2}+\left(v_{2}-v_{1}\right)^{2}\right)}
\end{aligned}
$$

With the help of the 3 vectors, $\vec{a}, \vec{b}, \vec{c}$ the projected location $\left(u_{i}, v_{i}\right)$ can be expressed by equation 3.3 .

$$
u\left(r_{i}\right)=\frac{\vec{a} \cdot \vec{r}_{i}}{\vec{c} \cdot \vec{r}_{i}+1} \quad v\left(r_{i}\right)=\frac{\vec{b} \cdot \vec{r}_{i}}{\vec{c} \cdot \vec{r}_{i}+1}
$$

From the 3 intermediate vectors $\vec{a}, \vec{b}, \vec{c}$, equations 3.3 and 3.1 above, directly describe the 3 scalars, $u_{s}, v_{s}, f$, and the 4 vectors, $\vec{s}, \hat{u}, \hat{v}, \hat{n}$, which give the sought after 9 geometric calibration parameters.

$$
\begin{gathered}
f=\epsilon_{f} \frac{\|\vec{a} \times \vec{c}\|}{\|\vec{c}\|^{2}}=\epsilon_{f} \frac{\|\vec{b} \times \vec{c}\|}{\|\vec{c}\|^{2}} \\
u_{s}=\frac{\vec{a} \cdot \vec{c}}{\|\vec{c}\|^{2}}+u_{c} \quad v_{s}=\frac{\vec{b} \cdot \vec{c}}{\|\vec{c}\|^{2}}+v_{c} \\
\hat{u}=\epsilon_{f} \frac{\vec{b} \times \vec{c}}{\|\vec{b} \times \vec{c}\|} \quad \hat{v}=-\epsilon_{f} \frac{\vec{a} \times \vec{c}}{\|\vec{a} \times \vec{c}\|} \quad \hat{n}=\hat{u} \times \hat{v}=-\epsilon_{f} s_{p} \frac{\vec{c}}{\|\vec{c}\|} \\
\vec{s}=\frac{-s_{p}}{\|\vec{c}\|}\left(\frac{\vec{a} \cdot \vec{c}}{\|\vec{a} \times \vec{c}\|} \hat{u}+\frac{\vec{b} \cdot \vec{c}}{\|\vec{b} \times \vec{c}\|} \hat{v}-\epsilon_{f} \hat{n}\right)
\end{gathered}
$$

The variable $\left(u_{c}, v_{c}\right)$ is the cone-beam projection of $\vec{r}=(0,0,0)$, the center of the phantom, onto the detector plane. The variables $s_{p}$ and $\epsilon_{f}$ are simply sign conventions based on the type of imaging system being calibrated, either pin-hole or cone-beam imaging. For our system $s_{p}=-1$ and $\epsilon_{f}=+1$.

Now, by using Eqn. 3.1 and the determined values of $u_{s}, v_{s}, f, \vec{s}, \hat{u}, \hat{v}, \hat{n}$, 
the projection of any point in 3D space (relative to the calibration frame) can be calculated and easily identified on the projection image.

The 3 scalars, $u_{s}, v_{s}, f$, and the vector, $\vec{s}$, (6 geometric calibration parameters) are given directly while the detector orientation, $(\theta, \phi, \eta)$ is calculated as follows:

$$
\theta=\arcsin \left(\hat{n}_{z}\right) \quad \phi=\arctan 2\left(\frac{\hat{n}_{x}}{\hat{n}_{y}}\right) \quad \eta=\arctan 2\left(\frac{\hat{u}_{z}}{\hat{v}_{z}}\right)
$$

where $\arctan 2$ is the four quadrant inverse tangent.

The 9 geometric parameters completely specify the scanner geometry however all the determined scalars and vectors are useful and stored for subsequent use.

\subsection{Geometric calibration implementation}

The calibration method was implemented using a Matlab program first written by Dr. Catherine Mennessier in France and also independently implemented here in Ottawa. This was done to validate and corroborate results. The programs had different methods of image segmentation and ball localization but the localization was the same within sub-pixel accuracy leading to virtually identical results. The most recent implementation of the geometric calibration which was used for the results presented in this study was done by myself and will be described below.

The geometric calibration phantom was imaged for a full $190^{\circ} \mathrm{scan}$, obtaining 100 projection images, see Fig. 3.5. An ImageJ [38] macro was used to automatically process the images and find the $(u, v)$ detector positions for the 6 balls of the calibration phantom in each projection. ImageJ found the locations of the balls by simply thresholding the image at a predefined level, converting the image to binary and then using a function designed to locate particles in an image. The macro specifies the particles' sphericity and approximate area to easily identify the balls. The sub-pixel location of the balls was determined by the center of mass location of the binary 


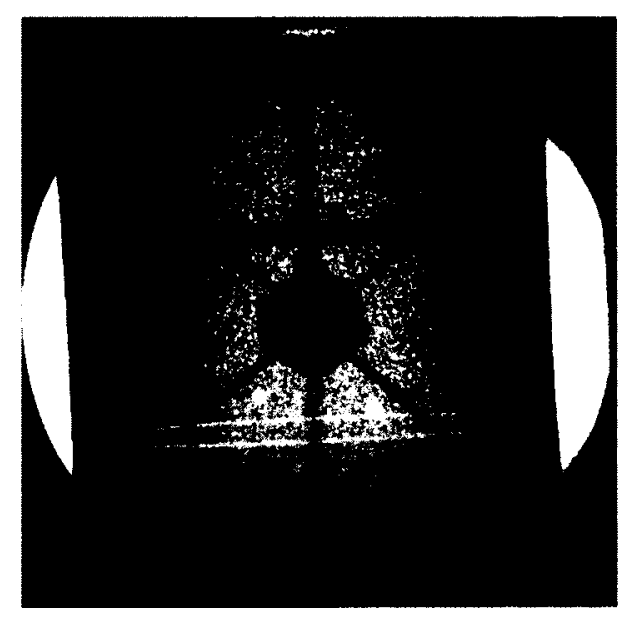

Figure 3.5: One of the projection images of the geometric calibration phantom suspended in a plastic cylindrical case.

image of the ball.

Due to the careful positioning of the phantom in the scanner in each projection image the distance each ball is from the mid-line of the phantom, the copper wire, adequately identifies the ball. This is certainly not the case if the phantom is misaligned. Consequently, the balls corresponding to $\pm k x, \pm k y, \pm k z$, were easily identifiable based on their vertical or y position in the image, see Fig. 3.5. When the phantom is aligned the balls which are highest and lowest in the image correspond to the $+k z=50 \mathrm{~mm}$ and $-k z=50 \mathrm{~mm}$ respectively.

From the 100 projection images of the 6 ball calibration phantom $600(u, v)$ ball locations were identified and stored to be read directly into the calibration program.

For each projection, using the $6(u, v)$ detector positions of the balls, (12 scalars), the 9 geometric parameters that completely specify the geometry of the scanner were automatically calculated with the direct mathematical formulas implemented in the Matlab program. The program also required that the image size and detector pixel size are known and input. The program calculated the 9 fundamental parameters but also calculated other related parameters needed for image reconstruction:

1. the focal length, $f$, or distance from the source to the detector - 1 scalar 
2. the projection of the source onto the detector, $(u s, v s)$ - 2 scalars

3. the vector from the source to the calibration phantom center, $\vec{s}-3$ scalars

4. the vectors $\hat{u}, \hat{v}, \hat{n}$, whose triple product determines the detector orientation

5. additionally the vector from the calibration object center to the detector, $\vec{c}-3$ scalars

These 18 parameters (which are all expressed in the calibration object frame) were stored to be later used for image reconstruction or co-registration. This was repeated for every projection of the scan.

\subsubsection{Overview}

The entire process of calibration required the use of an ImageJ macro for image segmentation and the determination of the $(u, v)$ ball location in the image and a Matlab program to determine the calibration parameters. The calibration program obtained and stored all 18 necessary parameters for reconstruction of all 100 projections of the scan in approximately 30 seconds. The process of scanner geometry calibration is fully automated such that the operator interaction time is relatively negligible.

\subsection{Results}

\subsubsection{Measurements of the calibration phantom}

Before geometric calibration could be achieved the dimensions of the phantom had to be measured precisely. The distance between the outer edges of the two balls along each axis was measured, $d_{a x i s}$, with a vernier caliper to within $\pm 0.01 \mathrm{~mm}$. The $\mathrm{k}$ value for each axis was determined as one half of this distance, $d_{a x i s}$, with the radius of a ball subtracted: $k_{x}=\left(d_{x} / 2\right)-r_{b}$. 
Table 3.1: X-ray calibration phantom measurements.

\begin{tabular}{|c|c|c|}
\hline & Measured phantom ball to ball distances & Design specification \\
\hline \hline$k_{x}(\mathrm{~mm})$ & $62.49 \pm 0.02$ & 62.50 \\
\hline$k_{y}(\mathrm{~mm})$ & $82.45 \pm 0.02$ & 82.50 \\
\hline$k_{z}(\mathrm{~mm})$ & $102.44 \pm 0.02$ & 102.50 \\
\hline
\end{tabular}

The measured ball to ball lengths, seen in table 3.1 , were $0.01-0.03 \mathrm{~mm}$ different than expected lengths from the machining process, which is within the desired machined precision of $\pm 0.05 \mathrm{~mm}$.

\subsubsection{Evaluation with and without distortion correction}

The calibration phantom was scanned using the standard protocol of 100 projections over $190^{\circ}$. The projections were corrected for distortion using the procedure described in chapter 2 above. Each projection was then processed to obtain 9 geometric parameters for the corresponding scanner position. These parameters were obtained in the reference frame defined by the phantom. Further, geometric calibration was performed on the original un-corrected image stack for two reasons. First, the trend and variation of the calibration parameters with distortion versus without provides an indication of the severity of angle dependent distortion and an indication of the importance of distortion correction. Secondly, the comparison confers a notion of the calibration's sensitivity to the $(u, v)$ locations of the imaged phantom balls.

The calibration parameters of the focal length, $f$, and the norm of the vector $\vec{s}$, $|\vec{s}|$ are shown in Fig. 3.6 with and without distortion correction. The plots show that values of the parameters obtained with the uncorrected (distorted) image vary over a much larger range than the parameters from the corrected image. The figures also show that the local fluctuations (point to point fluctuations) of the calibration parameters are comparable. 


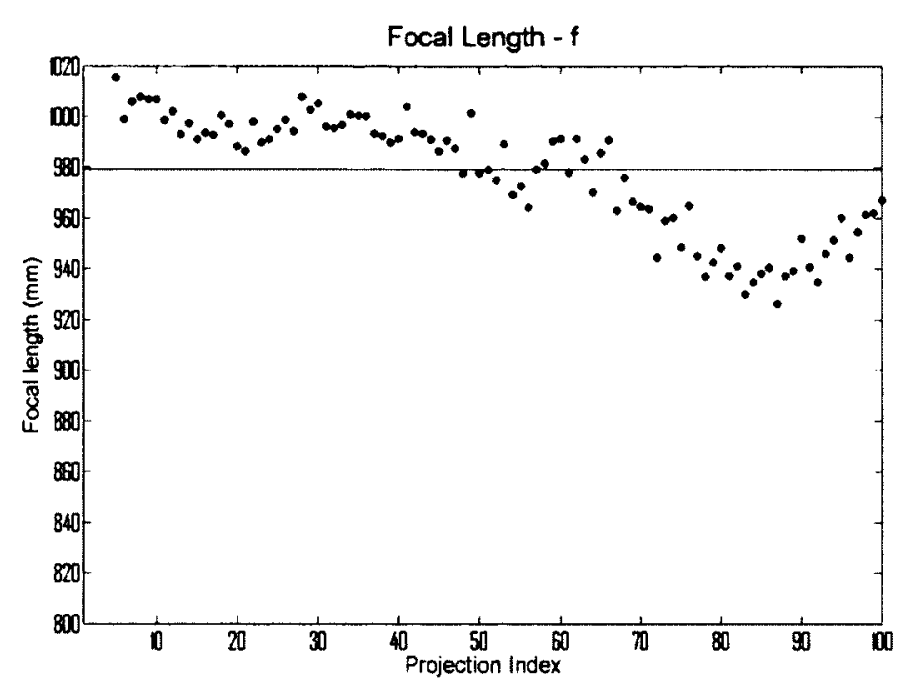

(a) Focal length with distortion correction

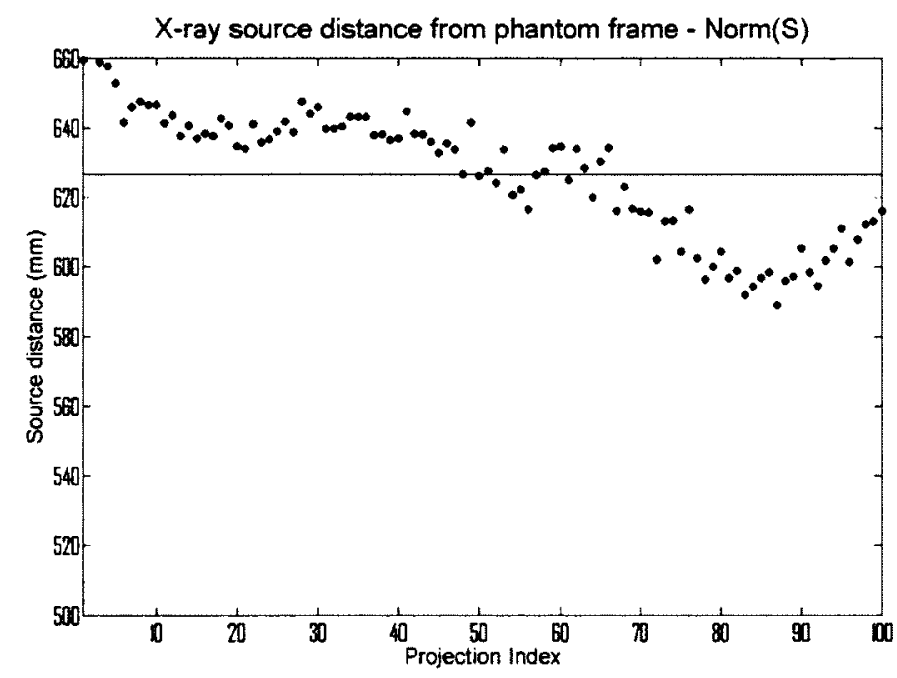

(c) Source Norm with distortion correction

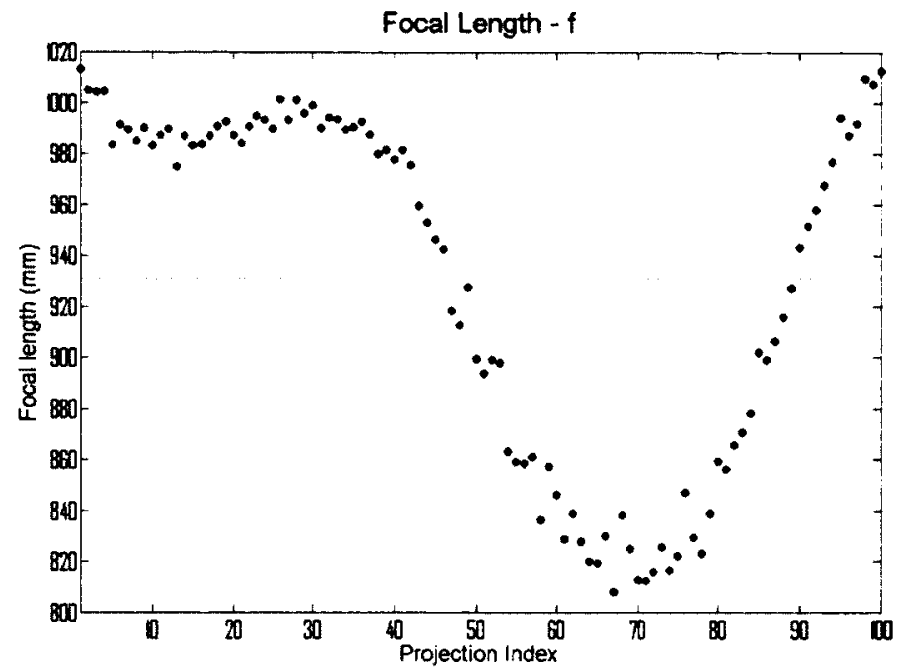

(b) Focal length without distortion correction

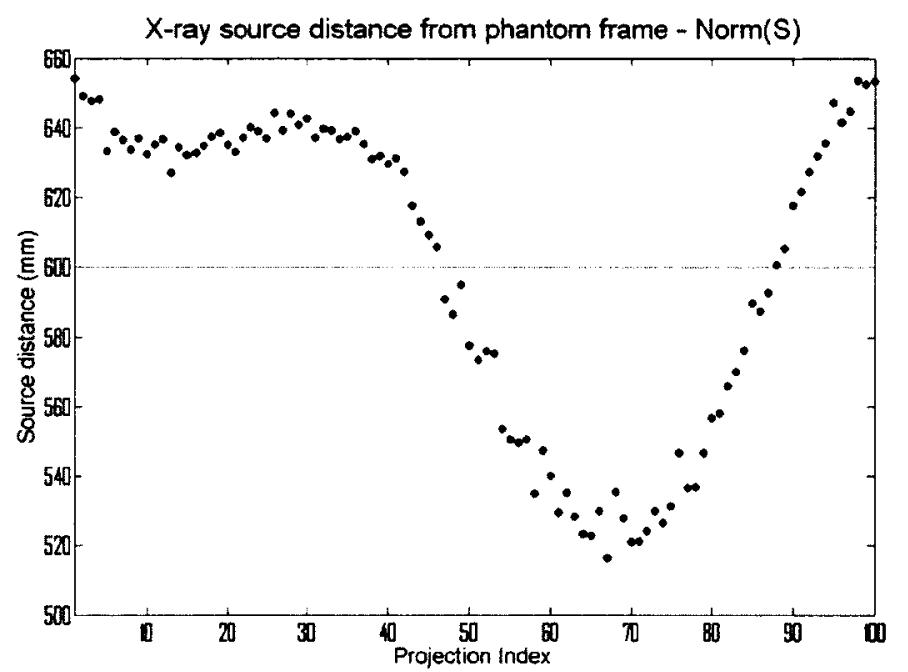

(d) Source Norm without distortion correction

Figure 3.6: Plots of geometric calibration parameters over projection index with and without distortion correction: (a) and (b) are plots of the x-ray source to detector distance, or the focal length, (c) and (d) are plots of the distance from the x-ray source to phantom.

The vector $\vec{s}$ represents the location of the x-ray source in the calibration object frame, therefore the norm of $\vec{s}$ would correspond to the distance from the calibration 
Table 3.2: Informal comparison of the listed and average values of the focal length and source to isocenter distance.

\begin{tabular}{|c|c|c|}
\hline Siemens Brochure & $\begin{array}{c}\text { focal length, } \\
f(\mathrm{~cm})\end{array}$ & $\begin{array}{c}\text { source to iso-center distance, } \\
|\vec{s}|(\mathrm{cm})\end{array}$ \\
\hline \hline $\begin{array}{c}\text { Avg. parameter value calculated } \\
\text { with distortion correction }\end{array}$ & 98 & 65 \\
\hline $\begin{array}{c}\text { Avg. parameter value calculated } \\
\text { without distortion correction }\end{array}$ & $93.1 \pm 6.9$ & $62.7 \pm 1.8$ \\
\hline
\end{tabular}

object (approximately the isocenter) to the source. The focal length is the distance from the source to the detector. These two values are listed by Seimens as: focal length, $f=98 \mathrm{~cm}$, and the source to isocenter distance, $|\vec{s}|=65 \mathrm{~cm}$ [31]. These values are approximate and considering both suffer considerably from effects such as gravity would vary during scanner rotation. Again assuming the calibration phantom is at the iso-center (such that the source to isocenter distance is the same as $|\vec{s}|$ ), an informal comparison can be made between the listed focal length and source to isocenter distance and the average of the calculated $f$ and $|\vec{s}|$, see table 3.2. The calculated average values of $f$ and $|\vec{s}|$ are given with their associated RMS uncertainty.

It is clear that the average calculated values with distortion correction of both the focal length and the source norm, $|\vec{s}|$, are closer to the values listed by Siemens. The actual bearing these comparisons have is limited.

\subsection{Discussion}

The calibration method successfully determined the 9 independent geometric parameters for each projection in the image data set. The parameters display not only large trends during the 100 projection scan but also a significant amount of local variation 
(fluctuations of $f$ on the order of $10 \mathrm{~mm}$ ). The scanner geometry is assumed to be imperfect during rotation, although the actual degree of this is unknown so a formal comparison of the trends of the calibration parameters with the theoretical scanner geometry is not possible. There are several factors that could disrupt the calibration.

The geometric calibration is understood to be sensitive to the imaged positions of the phantom balls as well as the physically measured phantom positions. The imaged ball locations have an associated error due to both the image thresholding to segment the ball locations and the determination of the center of the ball in the image. The center is determined using a binary center of mass weighting which is not exactly correct. The projection center of the ball could be more accurately estimated by using the center of the ellipse of the ball as described in [41]. These effects are estimated to be on the order of 0.1 pixels.

The residual error of the distortion correction is on average 0.63 pixels, which would likely be significant given the sensitivity of the calibration. It is known, however, that the correction accuracy is most accurate near the center of the image, and greatly degrades near the edges. All calibration phantom balls were located in the center of the image so the 0.63 average pixel error is an overestimate.

Additionally, the phantom construction and measurements could have corrupted the calibration. The phantom was machined such that the two balls on each axis should be equidistant from the center, such that $k_{x+}=-k_{x-}$. The calibration program heavily relies on this fact for accurate calibration, however, it was suspected that the distances may not be the same. Several different techniques were investigated to measure the distance from the center of the cube to each ball, however all proved to be ineffective at the accuracy required. The construction precision was on the order of $50 \mu \mathrm{m}$ which is $\sim 0.25$ pixels. These affects, though small, may have a significant impact on the geometric calibration. 


\subsubsection{Summary}

The comparison of the calibration parameters obtained with and without distortion correction clearly shows that angle dependent distortion strongly influences the calibration. The comparison further suggests that distortion negatively affects the calibration. As a result, angle dependent II distortion should be taken into account before attempting geometric calibration.

The calibration parameter trends and variations do not necessarily say if the calibration method was successful or not. It is not possible to deduce what the calibration parameters should look like based on our scanner. Furthermore, this research marks the first time this geometric calibration method has ever been applied to real data. In order to validate the geometric calibration method and evaluate its performance further steps must be taken. One such way to validate and evaluate the geometric calibration is through 3D image reconstruction, which is described in chapter 4 . 


\section{Chapter 4}

\section{Image Reconstruction and Evaluation}

In this project there are two main reasons to perform image reconstruction (IR). First, 3D image acquisition is required for PeTrack real-time tracking. Provided the imaging system and the Petrack system have been co-registered, the localization and tracking of positron markers can be accurately displayed on the 3D image. The Siemens system can also perform IR, however, the calibration employed to reconstruct these images is not accessible. A separate geometry calibration had to be performed to co-register PeTrack with the imaging system and reconstruct images for tracking. The PeTrack localization and tracking accuracy desired for this project is $\pm 0.5 \mathrm{~mm}$; therefore, if possible, the acquired images should have a similar accuracy.

Also, as described in chapter 3 , the acquisition of geometric parameters does not necessarily validate the calibration method; however, the successful reconstruction

of projection images using these parameters would conclusively validate the method using real data. Furthermore, the images obtained can be compared to images generated by the Siemens IR to evaluate not only the geometric calibration but also the benefits of angle dependent distortion correction. 


\subsection{Method}

\subsubsection{Image reconstruction theory}

The following describes the general principles of image reconstruction (IR) leading to the techniques used to generate images in this work.

Some basic principles will be described through examination of a simple case of reconstruction. The 2D reconstruction of a single slice of an object using data

obtained from a 1D fan beam as it rotates around an object can be used to illustrate basic computed tomography (CT) principles.

It is first imperative to understand what an x-ray projection image measures and what a reconstruction should show. An x-ray projection measures the intensity of x-rays which pass through the object and reach the detector, $I . I$ is a characteristic of the object material, and density, as well as the object thickness, the ray transversed to reach the detector. The following equation describes the relationship between the intensity of the measured ray and the object material and density, which is expressed by the material linear attenuation coefficient, $\mu$.

$$
I=I_{0} \exp \left(-\int_{\text {path }} \mu(x) d x\right) \Rightarrow \int_{\text {path }} \mu(x) d x=-\ln \left(\frac{I}{I_{0}}\right)
$$

The integration in the above equation is along the path of the ray and $I_{0}$ is the image intensity without an object in the path of the ray.

As indicated in Eqn. 4.1, the intensity of every recorded ray carries the information of $\mu(x)$ along the path of the ray. Hundreds of rays are measured for each projection and tens to hundreds of projections are obtained. The value of $\mu(x)$ of the object can be reconstructed through the process of backprojection.

There is another step which is essential for correct reconstructions, filtering. Due to the nature of backprojection, there is an over representation of the low spatial 
frequency component of the projection data and relatively an under representation of the high frequency component [42]. To account for this discrepancy the projection data must be filtered in the frequency domain to suppress the low frequency component of the projection data.

The general principles of $3 \mathrm{D}$ volume reconstruction can be explained by extending the $2 \mathrm{D}$ fan beam case. The same fan beam imaging used to obtain $2 \mathrm{D}$ reconstructions can simply be shifted up or down several times to obtain the reconstruction of several slices of the object to generate a $3 \mathrm{D}$ reconstructed volume [42]. The process of shifting the fan beam up or down many times and acquiring projection data is unfeasible as it would involve rotation around the object 100 times or more for even the simplest volume reconstruction. The inefficiency lead to the development of cone-beam imaging, which, as opposed to a 1D fan beam of x-rays, utilizes a cone of x-rays to measure a $2 \mathrm{D}$ projection image of the object [42]. The $2 \mathrm{D}$ projection would be an image of $n$ rows and $m$ columns which, in the fan beam case, would represent $n$ 1D fan beam projections of $m$ pixels in length.

To a good approximation, the $2 \mathrm{D}$ projection data can be filtered and back projected row by row to reconstruct the image. Instead of converting the 1D row of projection data to the frequency domain for filtering before converting back to the spatial domain for backprojection, to save computation time, the 1D row of projection data is simply convolved with a filtering kernel; a process that is mathematically identical [42].

The very complex process of filtering, backprojection, and volume reconstruction for cone-beam imaging can be completely handled using the Feldkamp algorithm [43]. The algorithm is approximate but useful, with relatively small errors. Advantages of the Feldkamp algorithm are that the reconstructions are managed analytically, it is specific for cone-beam imaging, and has relatively fast computation speed. The Feldkamp algorithm requires the precise knowledge of the scanner geometry for each 
projection of the scan. Moreover, the algorithm requires that the geometric calibration parameters be expressed in the source trajectory reference frame.

Additional steps must be performed in order to do short-scan cone-beam reconstruction. Standard cone-beam imaging involves rotating $360^{\circ}$ around the object which actually measures each ray through the object twice. Short-scan imaging, on the other hand, involves rotation of only $180^{\circ}+\delta$, where $\delta$ is the cone-beam angle. The addition of $\delta$ is a small correction factor to account for the diverging rays of the cone-beam. The short-scan reconstruction method must be modified to use Parker short-scan weights [44] to achieve reconstruction with the suppression of short-scan imaging artifacts.

In this study volume reconstruction was done analytically using the Feldkamp algorithm [43], modified to use Parker short-scan weights [44] to achieve short-scan cone-beam reconstruction.

\subsubsection{Re-expression of geometric calibration parameters}

The geometric calibration parameters are originally determined in the reference frame of the phantom, however, in order to be correctly used by the Feldkamp reconstruction algorithm they need to be re-expressed in the x-ray source trajectory reference frame.

The source trajectory frame must first be determined before the calibration parameters can be re-expressed in this frame. The geometric calibration calculates a source location, $\vec{s}$, in the reference frame of the phantom for each projection of the scan. The source trajectory is then determined as the "best circle" through all the x-ray source positions of the scan, see Fig. 4.1. The source trajectory frame was setup such that the best circle defines the $x, y$ plane, and the center of the circle defines the origin of the frame. The best circle is calculated using an orthogonal least squares method $[2,45]$. 


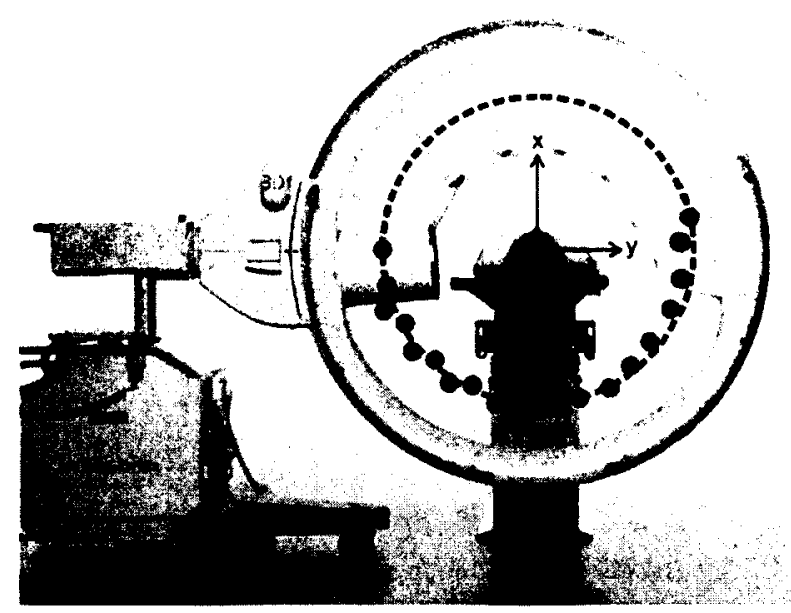

Figure 4.1: Diagram representing the source trajectory locations and the "best circle" determination of the source trajectory frame. (Diagram was adapted from [31])

Once the source trajectory reference frame has been determined all of the geometric calibration parameters need to be rotated to this frame and stored to be later used for image reconstruction.

\subsubsection{Reconstruction programs}

The image reconstructions in this study were done using a $\mathrm{C}$ program which had been previously written by Dr. Fred Noo. The program was operated as a "black box" in that only the input parameters were modified with no significant changes to the programing algorithm. The Hanning filter is used for filtered back projection.

The program employed to determine the source trajectory frame and re-express the calibration parameters was written by Dr. Catherine Mennessier, as a portion of her geometric calibration program.

This research was done in collaboration with Dr. Rolf Clackdoyle. 


\subsection{Reconstruction method application}

To accomplish full or short-scan reconstruction requires: the formatted projection images of the object (described in section 4.2.1), and an orbit file which specifies the exact cone-beam geometry and any other parameters needed for reconstruction (described in section 4.2.2). The orbit file was generated using the previously obtained geometric calibration parameters after they were re-expressed in the source trajectory frame (described above). Additionally, image reconstruction depended upon the input of a few known parameters of the scanner: image pixel size, image size, number of projections, approximate cone-beam radius (or source to detector distance/focal length) and then the desired size and resolution of the reconstructed image.

\subsubsection{Image formatting \& pre-processing}

The projection images to be reconstructed had to first be formatted to allow for the correct input into the IR program.

$\mathrm{C}$-arm image acquisition produces a stack of 100 projection images in Big Endian, unsigned 16 bit integer format. The stack of images remains in the same format after distortion correction.

The projection images needed to be formatted and saved in 32-bit real, little Endian format before input. Also, and more importantly, the images needed to be

preprocessed such that the projection data provided the measurement of $\int_{p a t h} \mu(x) d x$ instead of the intensity, $I$, see Eqn. 4.1 and section 4.1.1. To pre-process the images, first, the average background level in the image was measured, $I_{0}$, and the image stack was divided by that value. Subsequently, the natural log of each image was taken and the entire stack was multiplied by negative 1 . This converted the image into the necessary format required by the reconstruction algorithm.

An ImageJ [38] macro was again used to quickly convert the projection image 
stack to the necessary format for IR.

\subsubsection{Orbit file generation}

The orbit file is the means by which the image reconstruction algorithm determines which voxel (volume pixels) a pixel on a given projection image should be backprojected to. The orbit file format is specified by the IR program. For each projection image the orbit file presents 7 scalars ( 3 integers and 4 floats) and 5 vectors to the IR program. Some of the these may not be needed or not used for our IR. The input parameters are listed below and most are shown in Fig. 3.4.

1. Integer: discontinuity - not needed

2. Integer: neighbour - not needed

3. Float: smoothing - not used

4. Vector (3 floats): focus - synonymous to vector $\vec{s}$ from calibration

5. Vector (3 floats): tangent - not needed

6. Float: focal length, $f$ - supplied by calibration

7. Vector (3 floats): normal - synonymous to vector $\hat{n}$ from calibration

8. Vector ( 3 floats): $u_{\text {axis }}$ - synonymous to vector $\hat{u}$ from calibration

9. Vector (3 floats): $v_{\text {axis }}$ - synonymous to vector $\hat{v}$ from calibration

10. Float: $u_{\text {off }}$ - synonymous to vector $u_{s}$ from calibration

11. Float: $v_{o f f}$ - synonymous to vector $v_{s}$ from calibration

12. Vector ( 3 floats): center, $\vec{c}$ - supplied by calibration 


\section{Integer: $m_{\text {index }}-$ not needed}

A Matlab program is used to create the orbit file using the stored best circle rotated geometric calibration parameters. For all parameters of the orbit file which are not used or needed the value of zero was input. The orbit file can now be directly read into the reconstruction program.

\subsection{Evaluations}

Projection images of several phantoms have been acquired, corrected for distortion and reconstructed using the previously obtained geometric calibration parameters. The geometric calibration parameters were determined from distortion corrected images of the calibration phantom.

A high resolution image of the geometric calibration phantom has been reconstructed and shown in volume view representation to give an indication of the overall validity of the reconstruction(see Fig. 4.2). The figure can be visually compared with a photograph of the phantom in Fig. 3.3. The phantom was reconstructed using projection images with distortion correction as well as without correction to show the effects of distortion. These reconstructions were compared with the reconstruction done by the C-arm on-board system of the same scan. The slice view in the $\mathrm{x}$ direction of the three reconstructions is shown in Fig. 4.3 in which all reconstructions have the same voxel size. The effects of distortion can be clearly seen in both the reconstruction without correction and in the on-board Siemens reconstruction. The on-board reconstruction applied a heavier smoothing filter, and indicates some truncation compensation; seen as a bright ring at the edge of our image reconstructions. These differences prevent any quantitative comparisons with the Siemens on-board image reconstructions.

A planar resolution phantom was also scanned and reconstructed using projection 


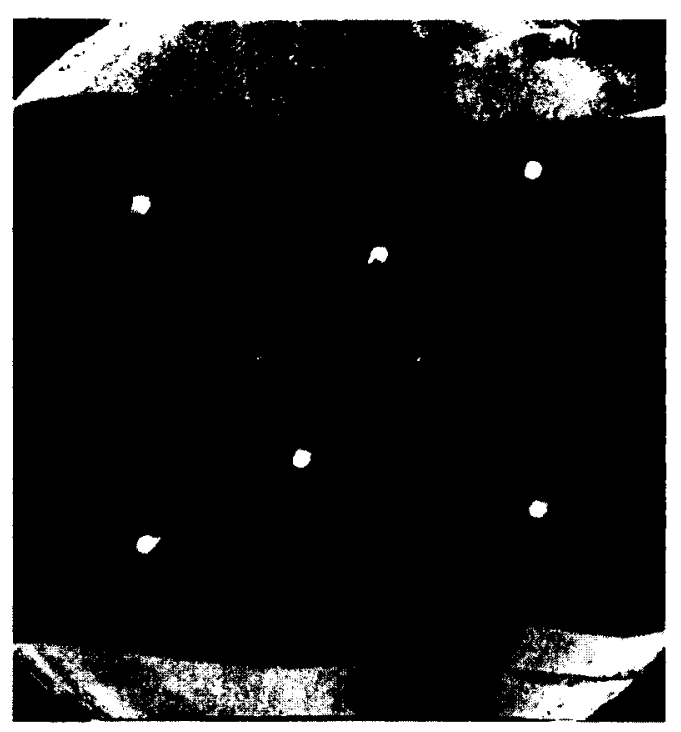

Figure 4.2: Volume view of a high resolution reconstruction of the geometric calibration phantom (Voxel size: $\left.(0.245 \mathrm{~mm})^{3}\right)$.

images both with and without distortion correction. Fig. 4.4 shows the slice view in the $\mathrm{x}$ direction of the phantom reconstructed with high resolution. The images in Fig. 4.4 are an average of a series of 23 sequential $x$ slices in order to show the full depth of the planar resolution phantom. The vertical resolution pattern does not appear to have much difference, however, the horizontal pattern and the clarity of the number scale shows improved quality in the reconstruction with distortion correction. Close inspection of the two images revaled that the $1.6 \mathrm{LP} / \mathrm{mm}$ were seen in the distorted image and $1.8 \mathrm{LP} / \mathrm{mm}$ were seen in the image with correction.

To provide a more quantitative evaluation of the reconstructed images with versus without distortion correction the image profile of the calibration phantom (Fig. 4.3), and the resolution phantom (Fig. 4.4), was measured along the indicated white line. The image profiles are plotted in figures 4.5 and 4.6. Both pairs of profile plots clearly show the quality of reconstructions without distortion correction suffers significantly. The profile plot with correction shows the wall of the aluminum tube and the background while the distorted image reconstruction does not. The actual 


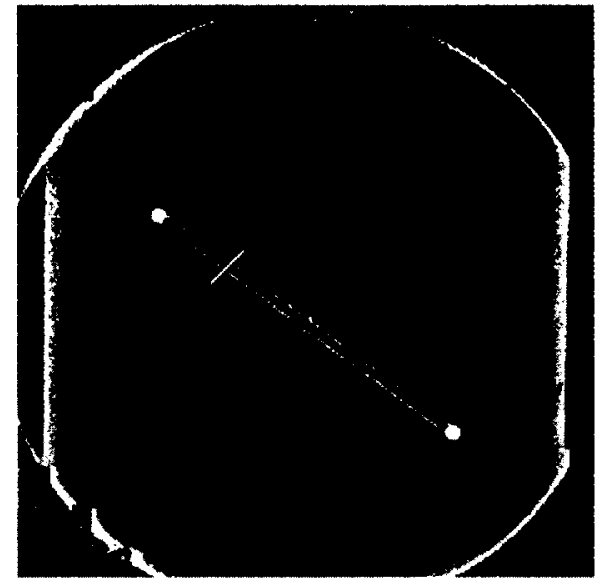

(a) Reconstruction with distortion correction

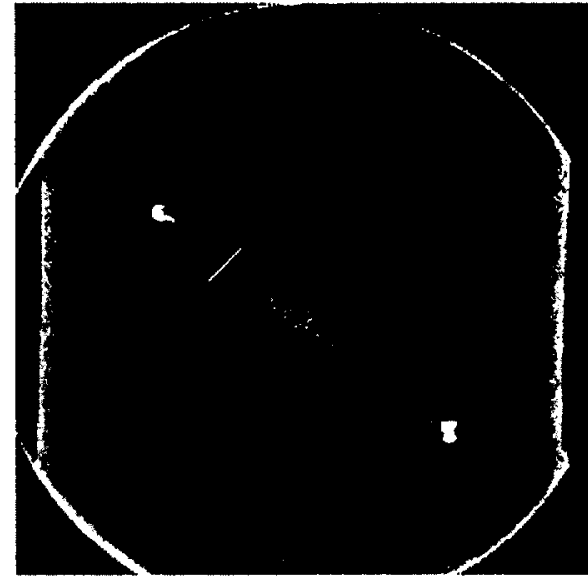

(b) Reconstruction without distortion correction

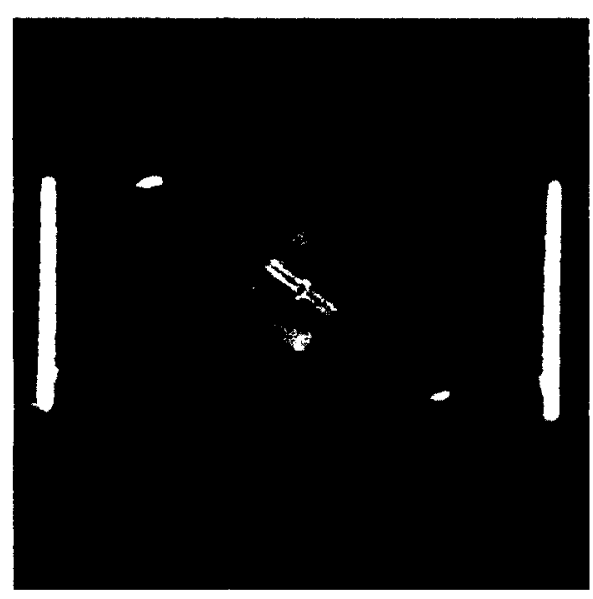

(c) Siemens on-board reconstruction

Figure 4.3: $x$ direction slice view of the reconstructed geometric calibration phantom (Voxel size: $\left.(0.49 \mathrm{~mm})^{3}\right)$. (Note: white line indicates location of profile plot - see Fig. 4.5)

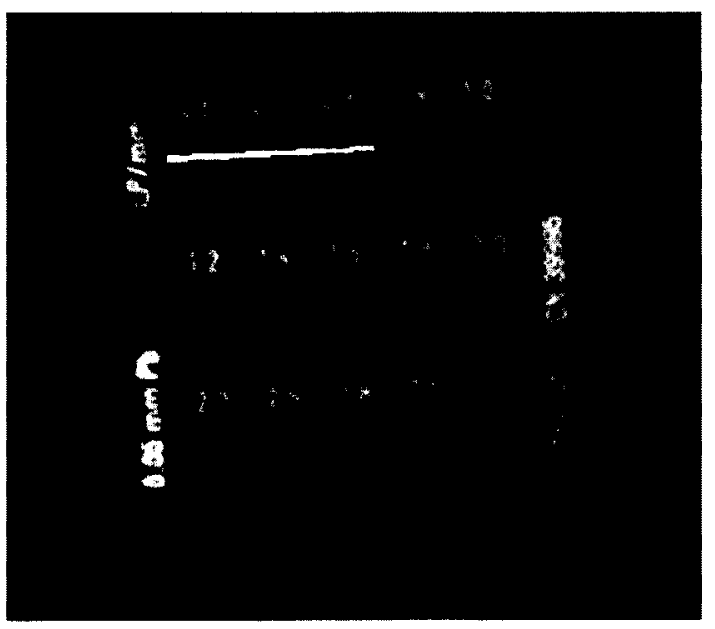

(a) Reconstruction with distortion correction

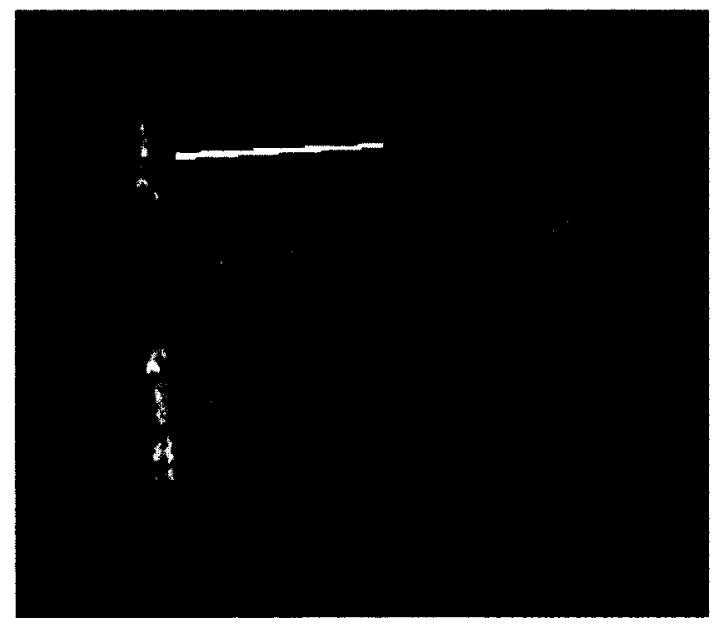

(b) Reconstruction without distortion correction

Figure 4.4: $x$ direction slice view of the reconstructed resolution phantom (Voxel size: $\left.(0.245 \mathrm{~mm})^{3}\right)$. (Note: white line indicates location of profile plot - see Fig. 4.6) 


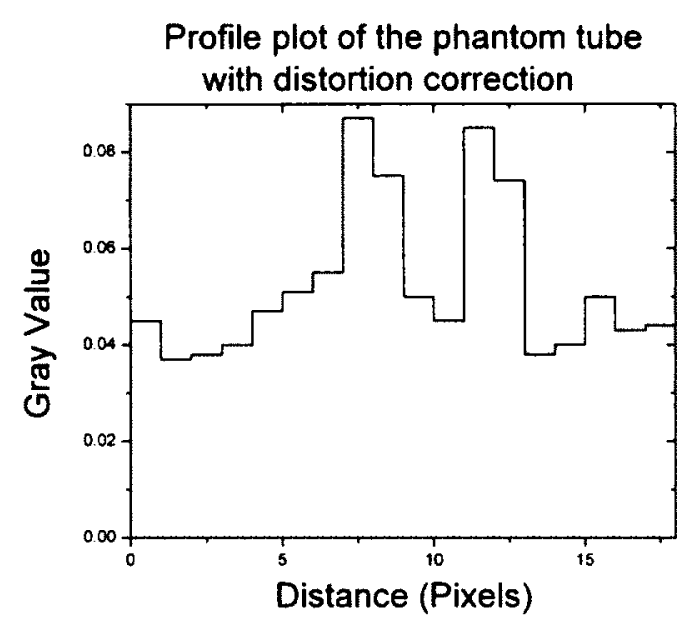

(a) Profile plot with distortion correction

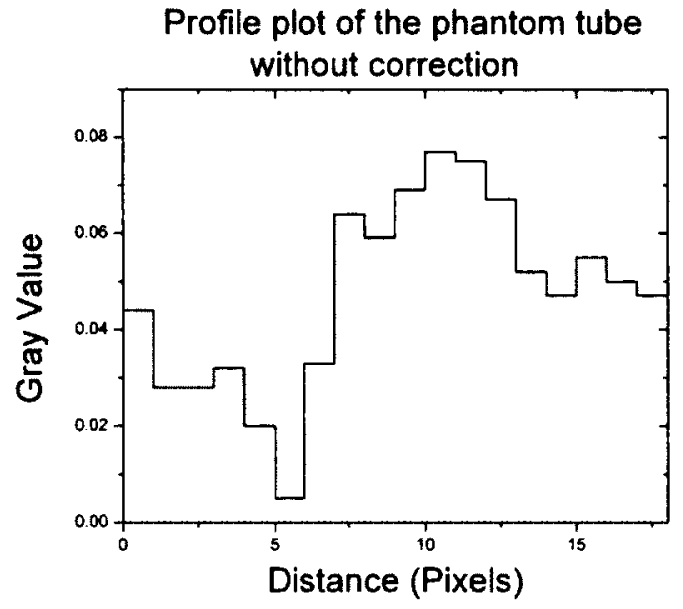

(b) Profile plot without distortion correction

Figure 4.5: Profile plot of the calibration phantom: (a) plot along the white line with distortion correction (Fig. 4.3(a), (b) plot along the white line without distortion correction (Fig. 4.3(b)

thickness of the aluminum tube is $0.355 \mathrm{~mm},(1.71$ pixels), which is the same thickness that is shown in the profile plot of the distortion corrected reconstruction. Figure $4.6 \mathrm{~b})$ clearly shows that the profile of the resolution grid is distorted.

A phantom composed of tissue like material was imaged and reconstructed to examine the quality of the reconstruction of a complex clinical object. Fig. 4.7 shows the slice view of the reconstruction performed with distortion correction and the on-board Siemens reconstruction. As before, the benefits of distortion correction are apparent, however, the Siemens reconstruction also shows signs of "C" shaped artifacts which are caused by an in-accurate geometric calibration [46].

Lastly, a hand phantom was imaged and reconstructed to give an indication of the quality of the reconstruction of a clinical object. The hand phantom shown in Fig. 4.8 is composed of the bones of a human hand encased in Lucite to mimic tissue. A slice of the hand phantom reconstructed with distortion correction and reconstructed by the Siemens on-board system is shown in Fig. 4.9(a) and (b). The distortion 


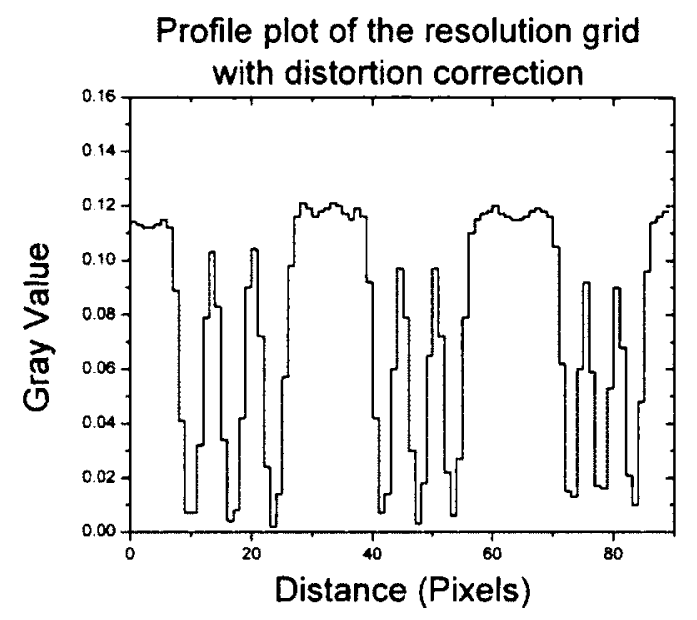

(a) Profile plot with distortion correction

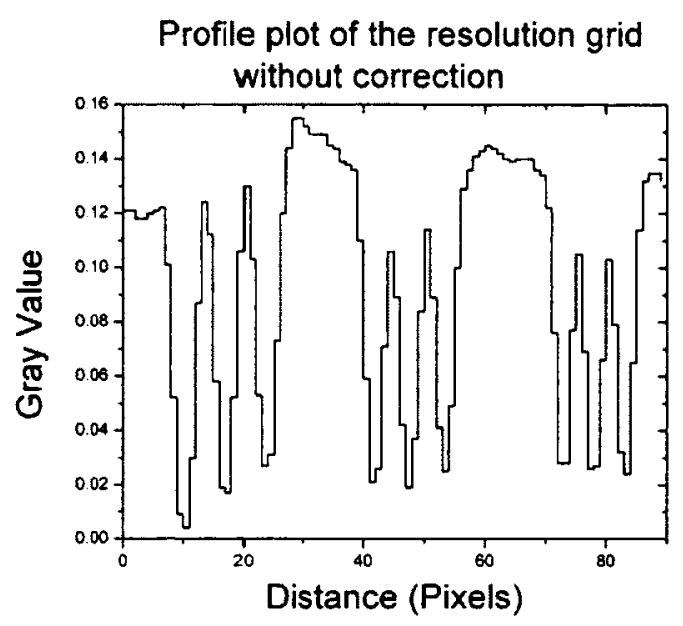

(b) Profile plot without distortion correction

Figure 4.6: Profile plot of the resolution grid phantom: (a) plot along the white line with distortion correction (Fig. 4.4a), (b) plot along the white line without distortion correction (Fig. 4.4b)

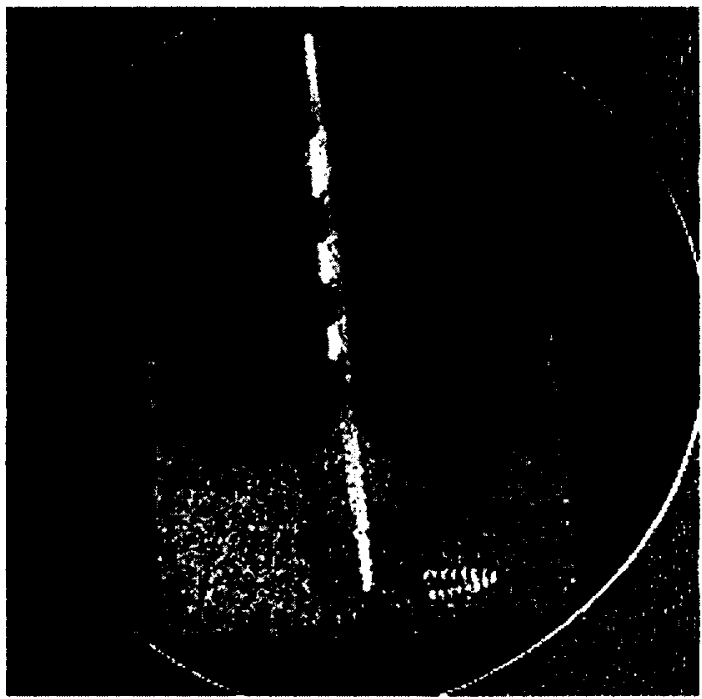

(a) Reconstruction with distortion correction

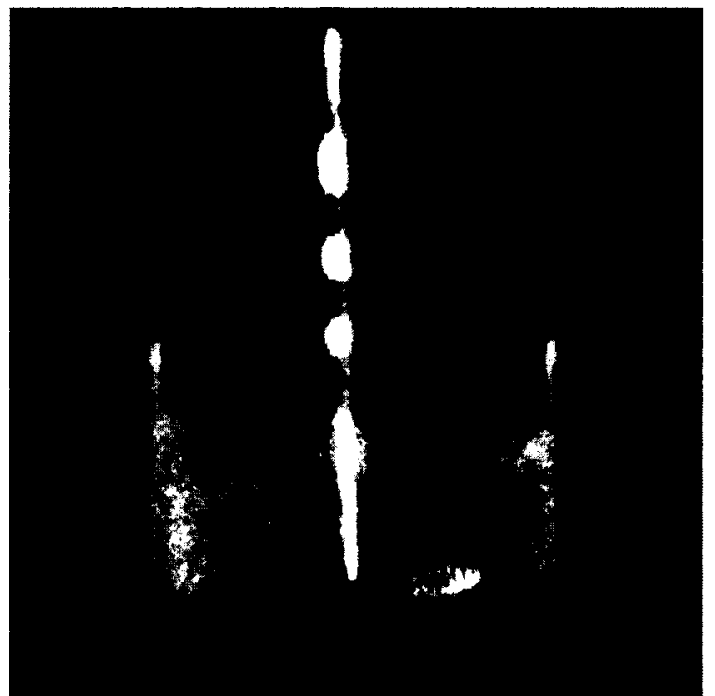

(b) Siemens on-board reconstruction

Figure 4.7: $\mathrm{z}$ direction slice view of quality phantom: (a) Reconstruction with distortion correction, (b) Reconstruction done by on-board system (Voxel size: $0.49 \mathrm{~mm}^{3}$ )) 
corrected reconstructed slice is observed to have more clearly defined edges around the bones while the Siemens reconstructed image is visibly smoothed. The same reconstructions can be seen in volume view in Fig 4.9(c) and (d). In these images the same view of all the slices of the hand phantom are displayed to show the entire bone structure of the hand. All volume view image parameters were identical between the two images except the threshold level which was slightly adjusted to show only bone with little to no tissue. Improved resolution is apparent as the intricacy of the bone structure is much more visible in the distortion corrected reconstruction. The truncation effects around the edge of the field of view, however, are prominent in the distortion corrected reconstruction, which is seen as a bright partial ring around the image. The Siemens on-board reconstructed image in volume view is again smoothed with less obvious truncation effects observed as blurring of the image around its edges.

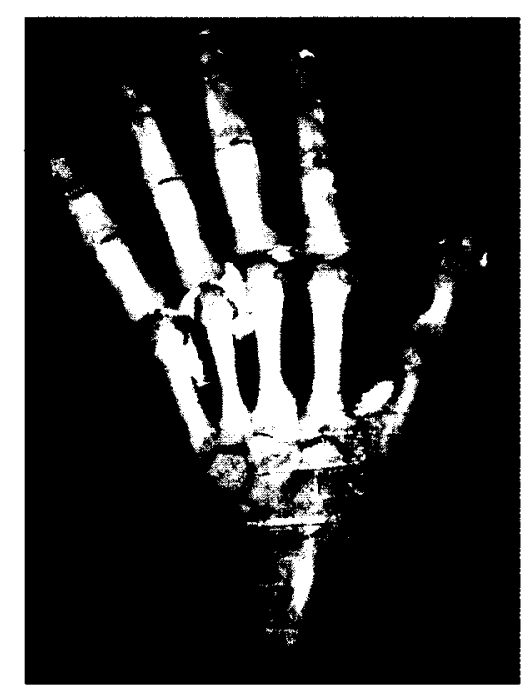

Figure 4.8: Hand phantom composed of bone encased in Lucite.

\subsection{Discussion}

The above image reconstruction validated the geometric calibration method for the first time with real data. Further, the above image comparisons quite possibly indicate 
that the geometric calibration performed in this study, using distortion corrected calibration images, operates as well if not better than the Siemens calibration.

The Siemens on-board reconstruction method implemented a heavy smoothing filter and truncation compensation which made it difficult to compare. Although, it was apparent that distortion affects could be seen in the Siemens image reconstructions and the reconstructions without correction. The evaluations have undoubtedly shown that distortion correction is required in order to achieve the best image quality. Our method of correcting for S-distortion is a process that the on-board Siemens system cannot do without a procedure to measure the local magnetic field strength affecting each individual scan. Consequently, improved resolution and the elimination of artifacts in the reconstructed images has been shown. Furthermore, the observed reconstructed resolution in the corrected resolution grid image, see Fig. 4.4, established that the resolution desired for PeTrack can be achieved.

Comparisons with Siemens reconstruction have in fact shown the need for truncation compensation around the edge of the field of view. These artifacts could possibly interfere with PeTrack performance in the future. 


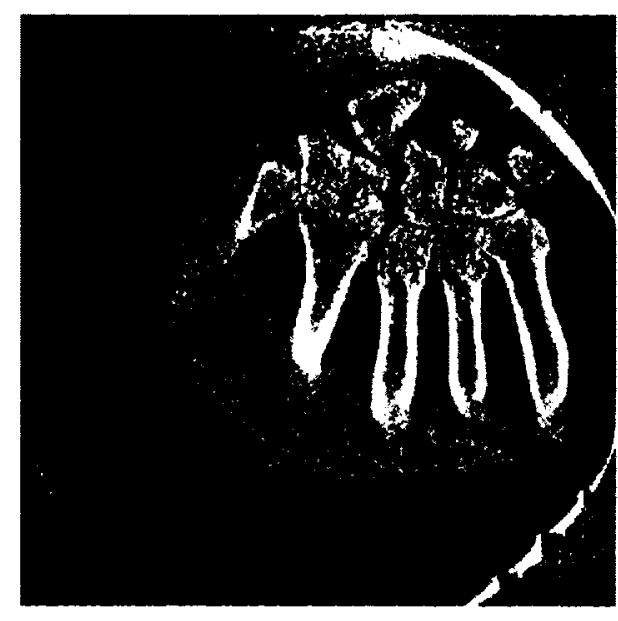

(a) Slice view reconstruction with distortion correction

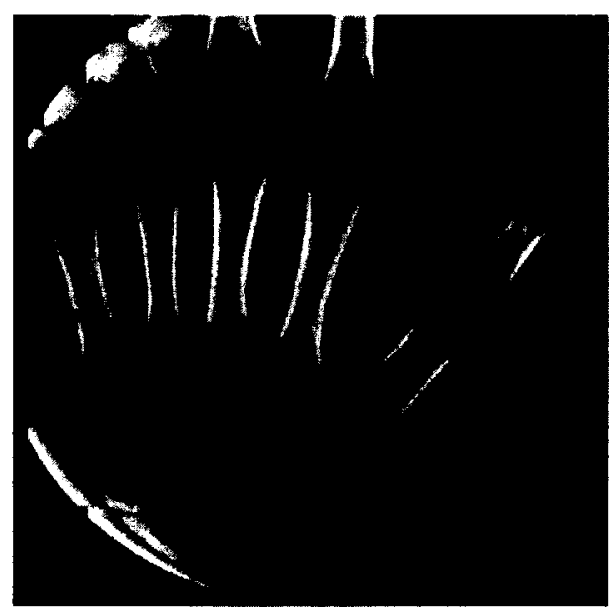

(c) Volume view reconstruction with distortion correction

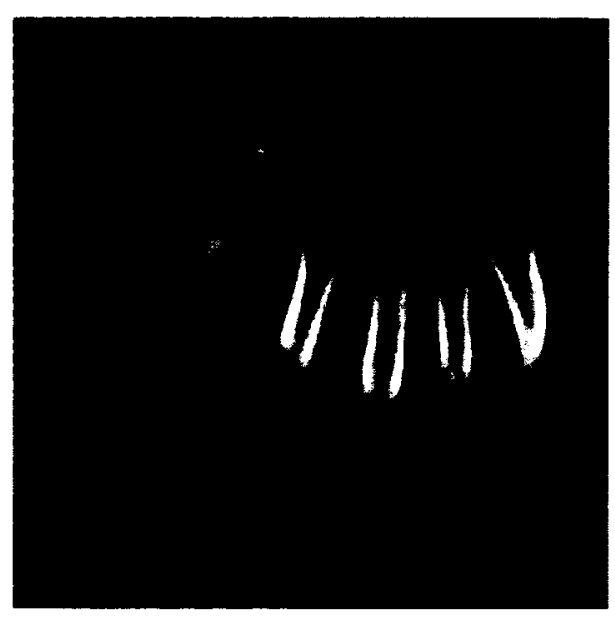

(b) Slice view Siemens reconstruction

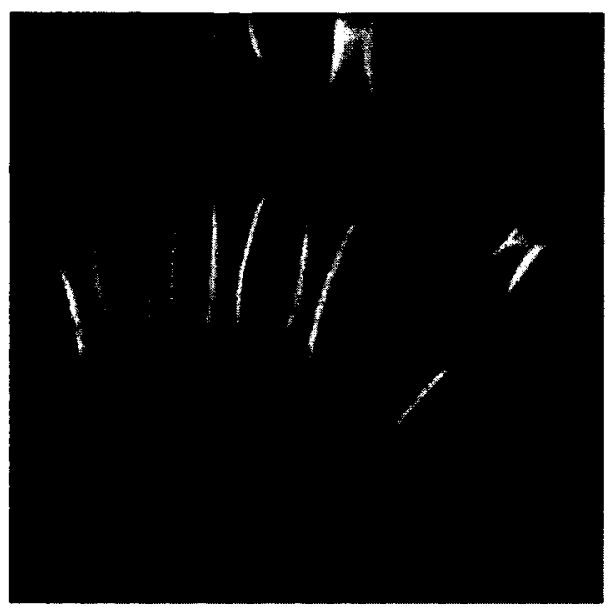

(d) Volume view Siemens reconstruction

Figure 4.9: Volume view and $x$ direction slice view of the reconstruction of the hand phantom (Voxel size: $(0.49 \mathrm{~mm})^{3}$ ). 


\section{Chapter 5}

\section{PeTrack: Prototype construction, Calibration \& Co-registration}

This chapter focuses on three main aspects of the development of the PeTrack system: the PeTrack prototype assembly and data acquisition, the development of a method of geometric calibration of the PeTrack system and then evaluation of this with a simulation study, and finally the co-registration of the PeTrack system with the x-ray imaging system with the use of a dual calibration phantom and some evaluations.

\subsection{Prototype construction}

The PeTrack system prototype contains: a light-weight stand to mount the detector modules onto and, four detector modules, which each contain 4 PMT detectors. Each module has an electronics system for data acquisition and a separate high voltage supply.

\subsubsection{Prototype assembly}

As described in section 1.3.1 each detector contains a BGO scintillation crystal, a PMT and a pre-amplifier in a light-tight housing. Each one of the detectors was 
carefully assembled by hand and involved many steps. The basics of the construction method and main components will be briefly described below with the help of figure 5.1 .

1. The detector housings were individually machined for our purposes. They are comprised of a black plastic case, an aluminum front cap, an aluminum end cap and a plastic screw.

2. The pre-amplifier was connected to the PMT.

3. The BGO crystal array was un-wrapped and cleaned with iso-propanol. A smooth coating of silicon grease was applied to one face of the BGO crystal array.

4. The PMT with connected pre-amplifier was firmly placed on the silicon grease on the BGO crystal array to remove trapped air bubbles between the grease and PMT face.

5. Without separating the PMT from the crystal, the PMT with connected preamplifier, BGO crystal, aluminum front cap, and end cap were carefully placed in the black plastic case before it was closed, securing all the components.

6. Aluminum tape was placed along the opening of the case to keep it closed and the plastic screw was fastened through the end cap into the preamplifier to further hold everything in place.

7. The entire detector was then securely placed into the module.

This was repeated for 16 detectors in the 4 modules. Each module has a separate high voltage power supply, a gating signal input and a data acquisition output, connected to each PMT of the module. 


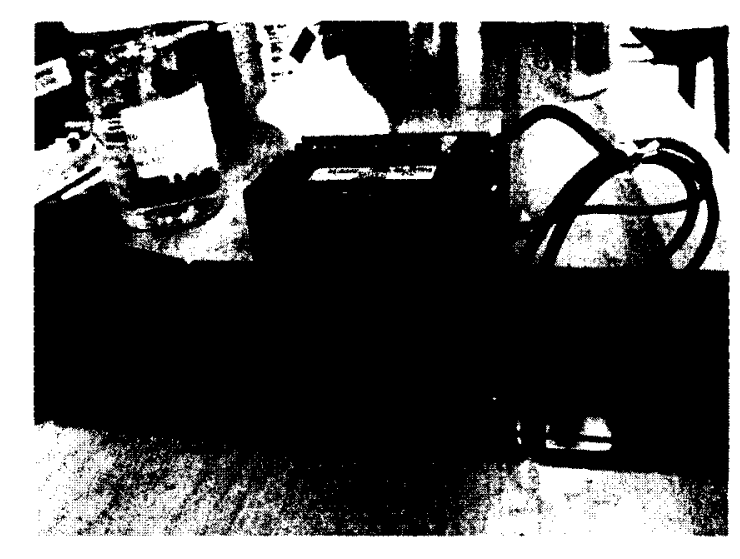

(a) PMT and black plastic case

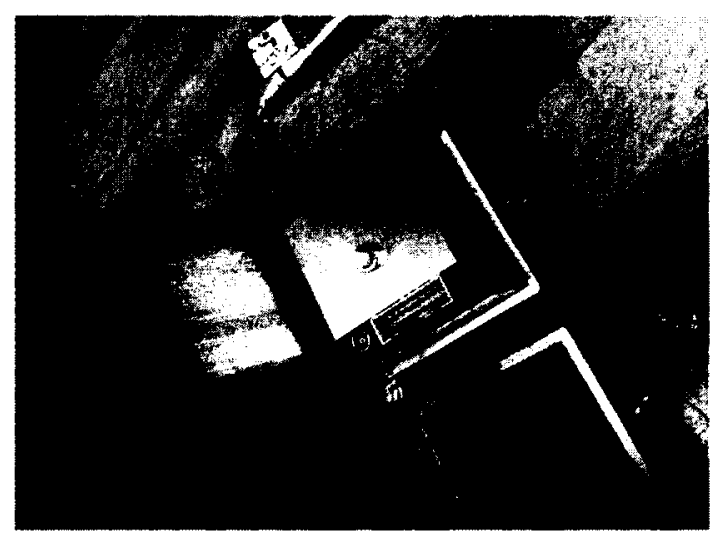

(c) Back view of constructed detector placed in detector module

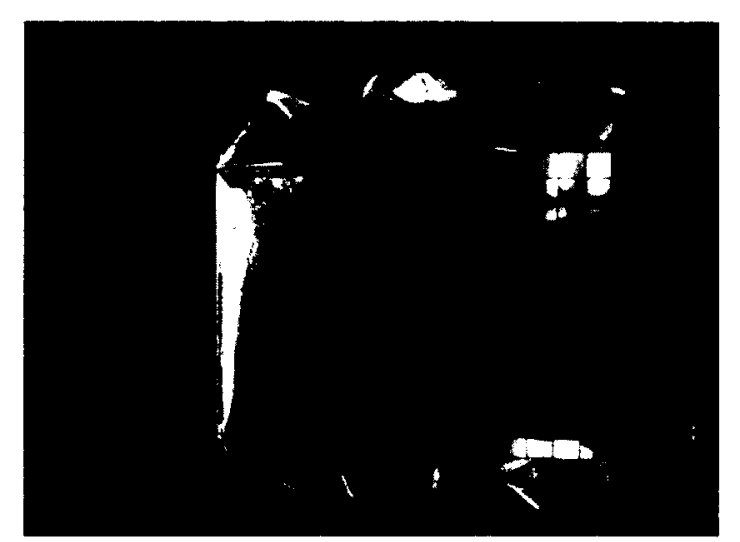

(b) BGO crystal array

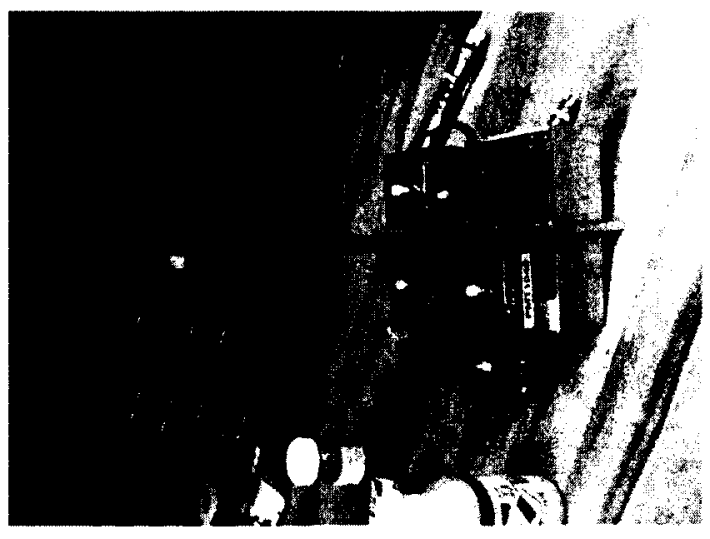

(d) Three detectors in a module

Figure 5.1: Various steps of PMT construction. 
The four modules were mounted on an aluminum supporting structure placed on the floor, see Fig 5.2. The structure can be adjusted to be extended or leveled based on the needs of the system. As well, each module can be moved vertically and rotated to a desired angle allowing proper alignment of the PeTrack system.

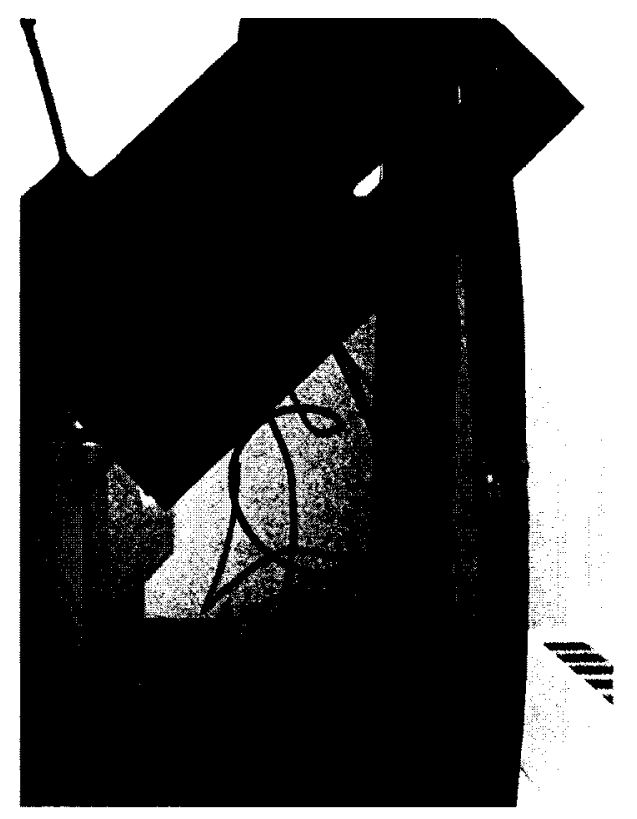

Figure 5.2: Detector module mounted on frame.

The constructed prototype along with the relevant electronics were then added to the C-arm imaging system, see Fig 5.3. With the help of a cross-line laser the PeTrack modules were aligned to face the C-arm isocenter with a $90^{\circ}$ angle between the two pairs of modules. This facilitates co-registration and tracking.

\subsubsection{Data acquisition}

The two pairs of modules are read out by MPET- 8 component. An intelligent central processing device (MCPD2) transmits the data from the MPET-8 readouts to a PC to be analyzed, see Fig. 5.4.

For each detected gamma ray, the readout system determines the following information: the energy of the detected gamma ray, the location the gamma ray was 


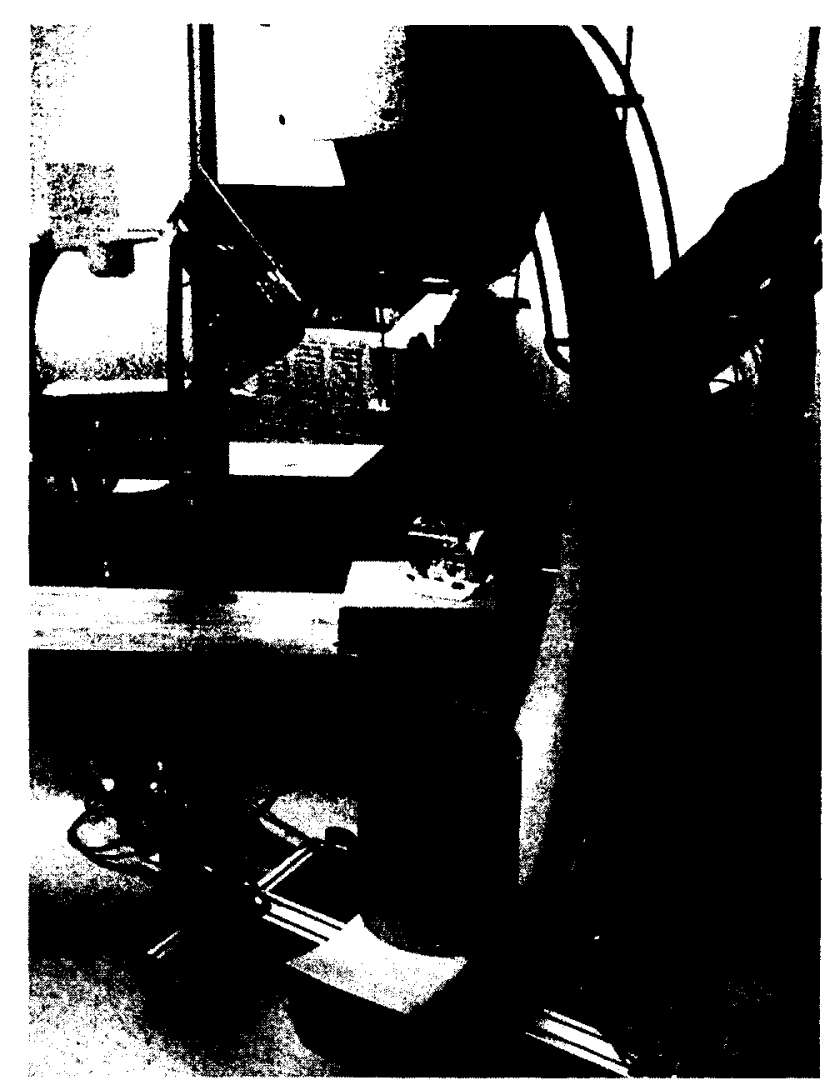

Figure 5.3: PeTrack detectors and structure.

detected on the position sensitive PMT, and the time the interaction occurred. The MPET-8 component receives the detection information from both detector modules of a pair. Based on the time of detection the MPET-8 determines which events from a pair of detector modules were likely the detection of two back-to-back gamma rays emitted as result of a positron annihilation, a coincidence event. The coincidence window of the system in $30 \mathrm{~ns}$. All other non-coincidence events are discarded.

The positions at which the two photons of a coincidence event interacted on the detector make the two end points of a coincidence line. Theoretically, the positron annihilation occurred at some point along this line. The coincidence lines are passed to the expectation-maximization clustering algorithm [26]. The number of positron sources being localized is assumed to be known by the algorithm. The algorithm sorts the coincidence events into a number of clusters that cross at close to the same 


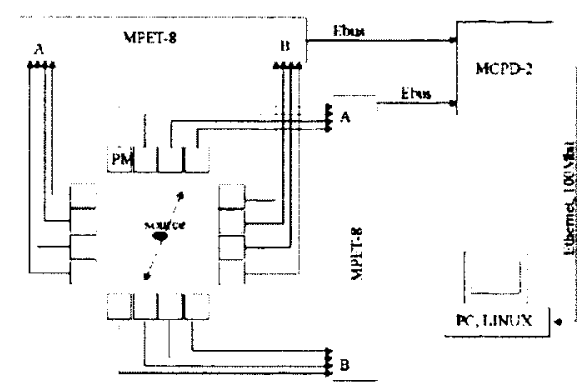

Figure 5.4: The MPET- 8 and MCPD2 electronics. (Source: The mesytec users manual [30])

locations based on the number of sources. After a given number of coincidence lines have been detected, the algorithm determines a source localization by determining the center of mass of all the detected coincidence lines of each cluster. The algorithm continually updates the source position as more coincidence data is acquired.

\section{PeTrack resolution}

There are three main factors which affect the accuracy of the detection of positron sources: positron range, non-collinearity of annihilation events, and scintillator crystal size of the detector.

When a positron is emitted from a source it is emitted with some amount of kinetic energy. It can more readily interact with matter and annihilate once it disperses this kinetic energy, hence once it slows down. Therefore most emitted positrons travel some distance away from the source while slowing down before they annihilate. The average distance traveled is called the positron range and will depend on the energy of the emitted positron and the material the source is in.

When a positron annihilates with an electron both particles will have some kinetic energy and momentum. This energy and momentum is transfered to the photons created in the annihilation causing them to be emitted at an angle from one another 
which deviates slightly from $180^{\circ}$. If the two photons are detected from this noncollinear annihilation the coincidence line between their detection locations would be incorrect under the assumption the annihilation was exactly collinear.

The PeTrack localization also relies on the exact location of the detected photons to be known. When the photon interacts in the scintillation crystal the actual location of interaction within the crystal is unknown and assumed to be at the center of the crystal. To reduce this detection uncertainty to a more reasonable amount the crystal is cut into small $4 \mathrm{~mm}$ square pieces. The photon interaction site in the crystal is thus known to about $\pm 2 \mathrm{~mm}$ accuracy.

These 3 factors which affect the accuracy of the detection of positron sources all contribute a random error to the source location. This random error causes coincidence lines to be determined at a random distance and direction away from the source location. The detected coincidence lines are assumed to be evenly distributed around the true source location due to this random error. Therefore, by simply using several coincidence lines to calculate the center of mass source location these random errors are significantly depreciated and the source location can be precisely determined. Given these factors a resolution of $0.5 \mathrm{~mm}$ should be attainable with the detection of less then 100 coincidence lines per second per positron source localization.

\subsection{PeTrack calibration}

PeTrack geometric calibration uses a phantom of known specifications to determine the exact location of the detector modules relative to the phantom frame. First, point sources are placed on the phantom at known positions relative to the phantom. Those sources are then tracked by the PeTrack system and determined in the PeTrack coordinate frame (Fig. 5.5) which is defined by the detectors. The relation between the tracked source locations in the PeTrack frame and the known locations in the 
phantom frame determines the mapping of all locations from the PeTrack frame to the phantom frame. The goal is to add sources to the already existing $x$-ray phantom to provide the common frame for co-registration from the PeTrack to the x-ray imaging frame.

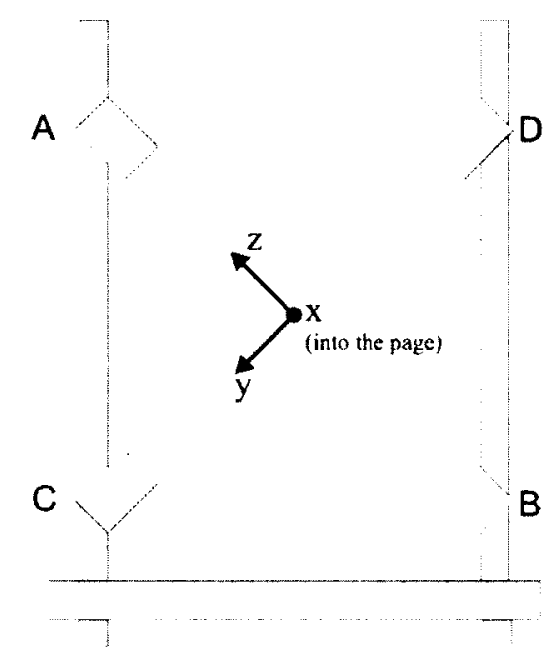

Figure 5.5: Illustration of the PeTrack coordinate frame.

\subsubsection{PeTrack geometric calibration}

It was decided that 4 sources were to be used for the PeTrack calibration. The sources were to be positioned in a similar way to the x-ray calibration phantom: 4 sources extending from the faces of a cube, two on one axis and one on each of the other two axes. The center of the cube defines the phantom reference frame.

The PeTrack system would determine $4(x, y, z)$ locations of the sources, which is 12 scalars. Therefore at most 12 scalars can be determined by the calibration method. Examination of the PeTrack system shows that there are actually 24 parameters required to completely specify it: an $(x, y, z)$ location for each detector and a $(\phi, \theta, \eta)$ detector orientation for each detector. 
Some information must be assumed or measured to minimize the number of parameters to be determined. After careful alignment of the detectors it is feasible to assume:

- that each pair of detector modules exactly face each other

- that each pair of detectors has a fixed distance $z$ between them, and they are not laterally shifted from each other

- the distance between the two modules in each pair can be measured

- the angle between the two pairs of gamma detectors is known

These assumptions leave only 6 parameters to find: a $3 \mathrm{D}$ position corresponding to the center of the detector system in the phantom frame, and a $3 \mathrm{D}$ rotation $(\phi, \theta, \eta)$ to define the rotation from the detector coordinate system to the phantom coordinate system.

From these 6 parameters a localization in the tracked frame can be re-expressed in the phantom frame. A source location would first be translated, to account for the translation between the detector frame and the phantom frame, and then a rotation matrix, given in Eqn. 5.1, would rotate any position in the tracked frame, $(x, y, z)$, to the phantom coordinate system, $\left(x^{\prime}, y^{\prime} z^{\prime}\right)$.

$\left[\begin{array}{l}x^{\prime} \\ y^{\prime} \\ z^{\prime}\end{array}\right]=\left[\begin{array}{ccc}\cos \theta \cos \eta & -\cos \phi \sin \eta+\sin \phi \sin \theta \sin \eta & \sin \phi \sin \eta+\cos \phi \sin \theta \cos \eta \\ \cos \theta \sin \eta & \cos \phi \cos \eta+\sin \phi \sin \theta \sin \eta & -\sin \phi \cos \eta+\cos \phi \sin \theta \sin \eta \\ -\sin \theta & \sin \phi \cos \theta & \cos \phi \cos \theta\end{array}\right]\left[\begin{array}{l}x \\ z\end{array}\right]$

To solve for the angles $(\phi, \theta, \eta)$ of the rotation matrix is, in general quite a difficult task. An easier more rigorous way to solve the rotation matrix is to simply determine 
the elements of a 3 by 3, 9 parameter matrix using 3 orthogonally placed sources, identified in both frames. The solution to this rotation matrix is now an over determined system which not only represents the rotation but also compensates for some inaccuracies in both the measured phantom and the PeTrack tracking system. This rotation matrix, as well as being more robust and easily solvable, does not require complex calculation of the angles $(\phi, \theta, \eta)$.

This geometric calibration implementation is described below.

\subsubsection{Implementation}

Geometric calibration is done off-line using a $\mathrm{C}++$ program. The program contains the PeTrack expectation-maximization clustering algorithm as it is designed for future integration into the tracking software for on-line calibration. In the on-line case the program would continually receive coincident event data from the 4 sources which would be immediately used for tracking and calibration. In the off-line case the program reads in data of the detected coincidence events of the 4 source phantom measured at an earlier time. Either case applies to the description below.

A phantom is constructed based on the x-ray calibration phantom so it can serve as a dual calibration phantom for co-registration. The 4 phantom source locations are known in the phantom frame and input. The sources are extended from four faces of a cube so an example of such a phantom could have the following source locations: $K_{x}=\left(k_{x}, 0,0\right), K_{y}=\left(0, k_{y}, 0\right), K_{+z}=\left(0,0, k_{z}\right), K_{-z}=\left(0,0,-k_{z}\right)$ as shown in Fig. 5.6 where $k_{z} \neq k_{x} \neq k_{y}$. The values of $k$ would be measured precisely relative to the center of the phantom.

The calibration program would start by first handing the input data to the tracking algorithm which would localize the 4 sources in the PeTrack frame. The 4 tracked locations, $\left(P_{1}, P_{2}, P_{3}, P_{4}\right)$ are in an arbitrary order so they need to be sorted to determine which locations correspond to $K_{+z}, K_{-z}, K_{x}, K_{y}$, respectively. 


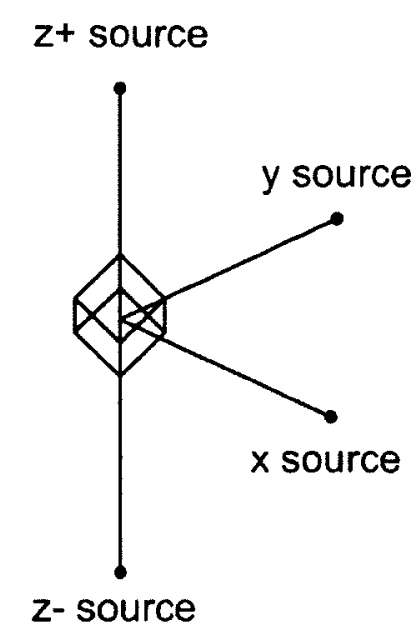

Figure 5.6: Illustration of the calibration phantom design.

Determining the arrangement of the sources is not a trivial task when their locations are given in an unknown reference frame. Information of the phantom and the values of $k$ must be utilized. The source arrangement is determined by realizing that given the 4 source locations there are 3 combinations of two line segments between the points: $P_{1} P_{2} \& P_{3} P_{4}, P_{1} P_{3} \& P_{2} P_{4}, P_{1} P_{4} \& P_{2} P_{3}$. Of these 3 combinations only one of them will have the property that the two line segments are perpendicular, hence their dot product is zero, or near zero. In the example above, see Fig. 5.6, this would correspond to the lines $K_{+z} K_{-z}$ and $K_{x} K_{y}$. The length of the line segments then determines which pair of points corresponds to the points on the $z$ axis and which points correspond to the $x y$ plane. The center of the phantom in the PeTrack frame was then determined as the middle of the projection points of $K_{x}$ and $K_{y}$ onto the line $K_{+z} K_{-z}$. The $K_{x}$ and $K_{y}$ points can now be determined by their unique distance from the center of the phantom. The $K_{+z}$ and $K_{-z}$ points can be differentiated using the right hand rule along with the $K_{x}$ and $K_{y}$ points.

The center of the phantom in the PeTrack frame was calculated in the above. The 
center is known in the phantom frame(which is usually $(0,0,0)$ ). The rigid translation between the two frames is then simply the difference between the center of the phantom in the two frames.

This translation is then directly applied to the tracked source locations before the next step of calibration. The rotation matrix to be determined is a 9 parameter, $a-i$, matrix. To accurately solve this matrix the 3 orthogonal source locations are required, hence only one of the two sources on the same axis is used. The matrix is defined as follows:

$$
\left[\begin{array}{l}
x_{1}^{\prime} \\
y_{1}^{\prime} \\
z_{1}^{\prime}
\end{array}\right]=\left[\begin{array}{lll}
a & b & c \\
d & e & f \\
g & h & i
\end{array}\right]\left[\begin{array}{l}
x_{1} \\
y_{1} \\
z_{1}
\end{array}\right]
$$

where $\left(x_{1}, y_{1}, z_{1}\right)$ is the location in the phantom frame and $\left(x_{1}^{\prime}, y_{1}^{\prime}, z_{1}^{\prime}\right)$ is the location in the PeTrack frame of source one of three. With the 3 known phantom locations, and the corresponding 3 known PeTrack source locations 3 separate matrices are obtained using Eqn. 5.2. By manipulating these 3 matrix equations, the following ensues:

$$
\left[\begin{array}{l}
a \\
b \\
c
\end{array}\right]=\left[\begin{array}{lll}
x_{1} & y_{1} & z_{1} \\
x_{2} & y_{2} & z_{2} \\
x_{3} & y_{3} & z_{3}
\end{array}\right]^{-1}\left[\begin{array}{l}
x_{1}^{\prime} \\
x_{2}^{\prime} \\
x_{3}^{\prime}
\end{array}\right]
$$

where $\left(x_{1}^{\prime}, x_{2}^{\prime}, x_{3}^{\prime}\right)$ are the three $x$ components of the source locations in the PeTrack frame. The parameters $d-f$ are solved using the $y$ components of the PeTrack source locations, $\left(y_{1}^{\prime}, y_{2}^{\prime}, y_{3}^{\prime}\right)$, and $g-i$ are solved with the $z$ components.

The matrix parameters, $a-i$, define a rotation from the phantom frame to the PeTrack frame. The inverse of this matrix defines the rotation from the PeTrack 
frame to the phantom frame.

The translation and the rotation matrix can be stored to re-express any subsequent source localizations in the phantom frame.

\subsubsection{Calibration simulation}

A simulation study was performed to test and validate the PeTrack geometric calibration using the GATE Monte Carlo package. The detector system was simulated assuming perfect geometry. A calibration phantom with 4 sources was designated as follows, (in mm): $(30,0,0),(0,40,0),(0,0,20),(0,0,-20)$. These 4 source locations were manually rotated and translated by user input amounts, see table 5.1. Four sources were positioned at these 4 new locations in the simulation. The simulation collected many coincidence events; while only a few hundred events are needed for localization, 4,000 coincidence events were handed to the tracking algorithm. This was done as a means to increase the accuracy of the tracking [22] and thereby increase the accuracy of the geometric calibration as a means to test and validate the calibration method while eliminating any PeTrack tracking effects.

The calibration procedure followed the description in section 5.2 .2 above to determine the calibration rigid translation and rotation matrix. The translated and rotated PeTrack source locations should correspond to the original phantom locations within the error of the PeTrack localization and geometric calibration.

Table 5.1 shows the true phantom location, the simulated translation, and simulated rotation angles, and then the position of the source locations after translation and rotation by Eqn. 5.1. Ten independent calibration runs were completed with the simulated data. The 10 runs were used to calculate the average and standard deviation values of the tracked location of the sources, the calculated rigid translation and

rotation and the location of the 4 sources after applying the calculated translation and rotation, shown in Table 5.2 . 
Table 5.1: True and simulated source locations after rigid translation and rotation.

\begin{tabular}{|c|c|c|c|}
\hline \multicolumn{4}{|c|}{ True phantom position } \\
\hline Source Position & $\mathrm{x}(\mathrm{mm})$ & $\mathrm{y}(\mathrm{mm})$ & $\mathrm{z}(\mathrm{mm})$ \\
\hline Source $\mathrm{x}$ & 30 & 0 & 0 \\
\hline Source $\mathrm{y}$ & 0 & 40 & 0 \\
\hline Source $\mathrm{z}+$ & 0 & 0 & 20 \\
\hline Source $\mathrm{z}-$ & 0 & 0 & -20 \\
\hline
\end{tabular}

\begin{tabular}{|c|c|c|c|}
\hline \multicolumn{4}{|c|}{ Simulated translation and rotation } \\
\hline Translation/Rotation & $\mathrm{x}(\mathrm{mm}) / \phi\left(^{\circ}\right)$ & $\mathrm{y}(\mathrm{mm}) / \theta\left(^{\circ}\right)$ & $\mathrm{z}(\mathrm{mm}) / \eta\left(^{\circ}\right)$ \\
\hline Translation & -3.0 & 5.0 & 2.2 \\
\hline Rotation & 45 & 15 & 60 \\
\hline
\end{tabular}

\begin{tabular}{|c|c|c|c|}
\hline \hline \multicolumn{4}{|c|}{ Source locations after translation and rotation } \\
\hline Source Position & $\mathrm{x}(\mathrm{mm})$ & $\mathrm{y}(\mathrm{mm})$ & $\mathrm{z}(\mathrm{mm})$ \\
\hline Source $\mathrm{x}$ & 17.08 & 20.10 & -9.96 \\
\hline Source $\mathrm{y}$ & -17.83 & 15.48 & 25.12 \\
\hline Source $\mathrm{z}+$ & 17.08 & -8.90 & 11.46 \\
\hline Source $\mathrm{z}-$ & -11.08 & -1.10 & -15.86 \\
\hline
\end{tabular}


Table 5.2: PeTrack localization of simulated source locations and the determination and application of a rigid translation and rotation calibration.

\begin{tabular}{|c|c|c|c|}
\hline \multicolumn{5}{|c|}{ PeTrack determined source locations } \\
\hline Source Position & $\mathrm{x}(\mathrm{mm})$ & $\mathrm{y}(\mathrm{mm})$ & $\mathrm{z}(\mathrm{mm})$ \\
\hline Source $\mathrm{x}$ & $17.42 \pm 0.08$ & $19.95 \pm 0.05$ & $-9.89 \pm 0.05$ \\
\hline Source $\mathrm{y}$ & $-17.71 \pm 0.07$ & $15.40 \pm 0.07$ & $25.08 \pm 0.06$ \\
\hline Source z+ & $17.03 \pm 0.08$ & $-8.80 \pm 0.04$ & $11.41 \pm 0.03$ \\
\hline Source z- & $-10.97 \pm 0.05$ & $-1.06 \pm 0.05$ & $-15.81 \pm 0.04$ \\
\hline \multicolumn{4}{|c|}{ Determined translation and rotation } \\
\hline Translation/Rotation & $\mathrm{x}(\mathrm{mm}) / \phi\left(^{\circ}\right)$ & $\mathrm{y}(\mathrm{mm}) / \theta\left(^{\circ}\right)$ & $\mathrm{z}(\mathrm{mm}) / \eta\left(^{\circ}\right)$ \\
\hline Translation & $-3.08 \pm 0.07$ & $4.94 \pm 0.04$ & $2.15 \pm 0.07$ \\
\hline Rotation & $44.80 \pm 0.12$ & $14.95 \pm 0.13$ & $59.20 \pm 0.25$ \\
\hline Source locations after translation and rotation \\
\hline Source Position & $\mathrm{x}(\mathrm{mm})$ & $\mathrm{y}(\mathrm{mm})$ & $\mathrm{z}(\mathrm{mm})$ \\
\hline Source $\mathrm{x}$ & 30.000 & 0.000 & 0.000 \\
\hline Source y & 0.000 & 40.000 & 0.000 \\
\hline Source z+ & 0.000 & 0.000 & $19.86 \pm 0.21$ \\
\hline Source z- & 0.000 & 0.000 & -20.000 \\
\hline
\end{tabular}


Given that the three source positions $(x, y$, and $z-)$ were used to determine the coefficients of the rotation matrix, when the sources are rotated into the phantom frame they should be exactly at their ideal locations which is in fact observed. The $z+$ source position, on the other hand, will not be translated and rotated exactly to it's ideal location. Therefore this source locations deviation from it's ideal location and it's uncertainty illustrate the accuracy of the calibration.

\subsection{PeTrack co-registration}

The method of co-registration between the x-ray imaging and PeTrack localization systems can finally be evaluated and discussed. The co-registration is fairly simple at this point, a dual x-ray/PeTrack calibration phantom is required and the calibrations are completed as described in section 3.2 for $\mathrm{x}$-ray calibration and section 5.2 for PeTrack calibration.

\subsubsection{Dual x-ray/PeTrack calibration phantom}

To accommodate the needs of the PeTrack system the previous x-ray calibration phantom design had to be modified and a new phantom was constructed. The phantom contains 6 steel balls for x-ray calibration with the addition of 4 positron point sources for PeTrack calibration.

The $\mathrm{x}$-ray calibration phantom was constructed as before except the $k_{z}$ value of the new phantom was decreased by $30 \mathrm{~mm}: k_{x}=30 \mathrm{~mm} k_{y}=40 \mathrm{~mm} k_{z}=20 \mathrm{~mm}$. As before, the x-ray calibration was comprised of 6 steel balls extended by aluminum tubes from the faces of a cube.

Each positron source is enclosed in a $3 \times 3 \times 8 \mathrm{~mm}^{3}$ acrylic housing. The active volume is $1 \times 0.5 \times 0.5 \mathrm{~mm}^{3}$. The positron sources were added to the $\mathrm{x}$-ray phantom by securing them over the already in place aluminum tubes with the sources extended 
approximately $4 \mathrm{~mm}$ past the steel balls. A carbon fiber tube and another aluminum tube were used to secure the positron source onto the phantom, see Fig. 5.7. The source tubes are placed over the carbon fiber tubes and not secured in order to allow the sources to be removed from the phantom when needed.

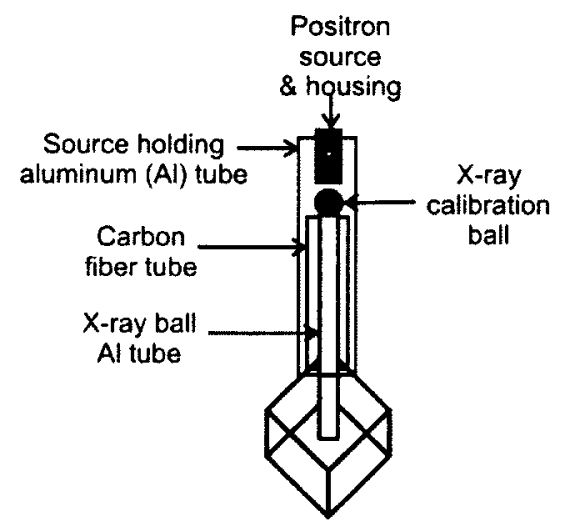

Figure 5.7: Schematic of the dual calibration phantom design.

As the sources must be extended from the cube farther than the steel balls the x-ray calibration phantom had to be modified to ensure the sources would not be farther than $5 \mathrm{~cm}$ from the center of the cube. The PeTrack field of view is on the order of $(10 \mathrm{~cm})^{3}$ and therefore the sources should not exceed $5 \mathrm{~cm}$ from the center of the phantom as they would become difficult, if not impossible, to localize.

As a last step a phantom holder was constructed for easy placement and manipulation, see Fig. 5.8 .

The $\mathrm{x}$-ray geometric calibration is very sensitive to the location of the phantom balls. The x-ray phantom was machined precisely such that the ball locations were known to within $\pm 50 \mu \mathrm{m}$. The PeTrack calibration, on the other hand, is not as sensitive to the position of the sources and they only had a desired accuracy of \pm 0.5 $\mathrm{mm}$. The exterior ball to ball distances of the x-ray phantom were measured for each axis and can be seen in table 5.3. The $k$ values of the $\mathrm{x}$-ray phantom were simply 


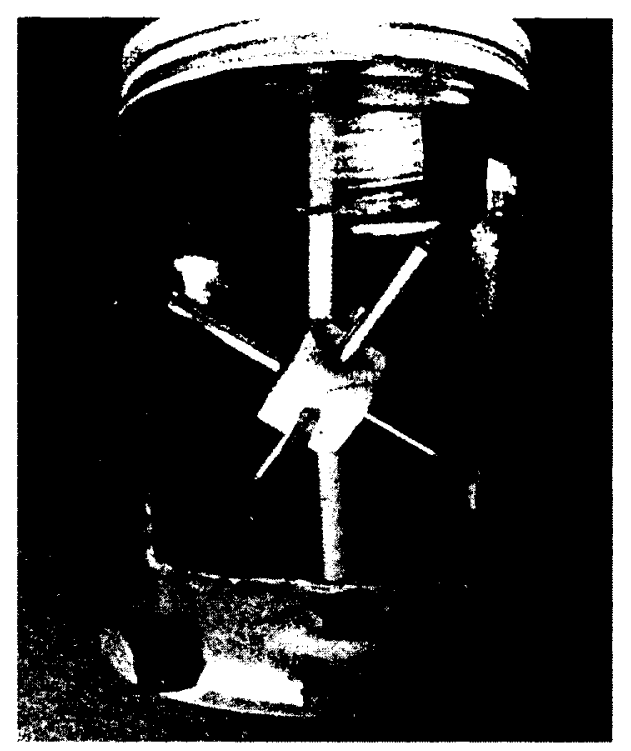

Figure 5.8: Picture of the Phantom and container.

Table 5.3: Measurements of the x-ray markers exterior ball to ball length

\begin{tabular}{|c|c|}
\hline Measured axis & Length $(\mathrm{mm})$ \\
\hline \hline $\mathrm{x}$ axis & $62.35 \pm 0.02$ \\
\hline $\mathrm{y}$ axis & $82.41 \pm 0.02$ \\
\hline $\mathrm{z}$ axis & $42.41 \pm 0.02$ \\
\hline
\end{tabular}

half of the exterior ball to ball distance. The locations of the positron sources were determined based on the $\mathrm{x}$-ray phantom ball to ball measurements and the length of the housing of the source $(8.1 \pm 0.1 \mathrm{~mm})$, see table 5.4 . The active volume of the source was assumed to be at the middle of the source housing.

\subsubsection{Co-registration method}

As described above, the PeTrack system was combined with the $\mathrm{x}$-ray system and aligned to the center of the $x$-ray phantom iso-center with a cross line laser. The dual calibration phantom was then placed in the field of view of both systems and aligned with the C-arm system for calibration imaging. The phantom was imaged for a full 
Table 5.4: Positron marker $k$ values calculated based on the $\mathrm{x}$-ray marker measurements and source housing length.

\begin{tabular}{|c|c|}
\hline$k$ value & Length $(\mathrm{mm})$ \\
\hline \hline$k_{x}$ & $35.2 \pm 0.1$ \\
\hline$k_{y}$ & $45.3 \pm 0.1$ \\
\hline$k_{z+}=k_{z-}$ & $25.3 \pm 0.1$ \\
\hline
\end{tabular}

100 projection $190^{\circ}$ scan and tracked by the PeTrack system, see Fig. 5.9.

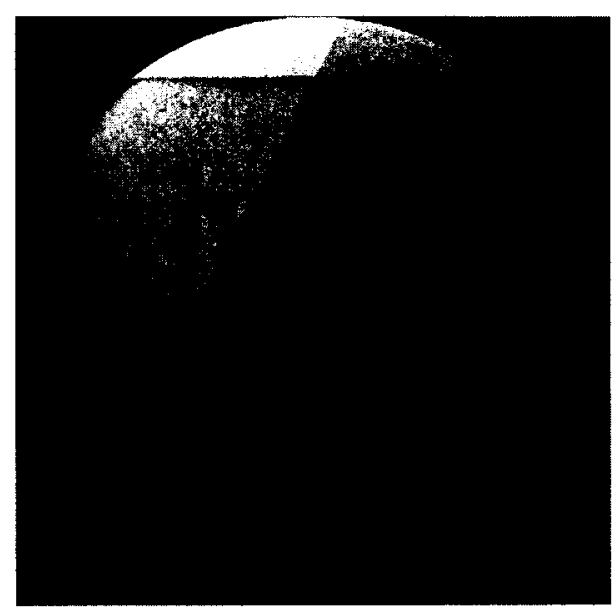

Figure 5.9: Projection image of the dual calibration phantom.

The 100 projections of the scan were corrected for distortion before the x-ray geometric calibration was done. The calibration determined and stored the parameters described in section 3.2.2, for every projection of the scan. The parameters were determined in the phantom frame. The projection of any $3 \mathrm{D}$ point (expressed in the reference frame of the phantom) onto the detector is described by Equation 3.3 and the determined calibration parameters.

The PeTrack system localized the 4 markers of the geometric calibration phantom in the PeTrack frame. The PeTrack geometric calibration determined the rigid translation and the rotation matrix and stored these parameters.

The two calibrations co-registered the systems. As long as the two calibrations 
are applicable, for instance neither system has moved (with the exception of the Carm gantry motion), sources localized and tracked by PeTrack can be translated and rotated into the phantom frame and then the $\mathrm{x}$-ray calibration parameters can be used to determine the tracked source locations on any acquired image.

\subsubsection{Co-registration evaluation}

The co-registration was evaluated by means of a static method of projecting source locations onto a $2 \mathrm{D}$ projection image. The sources were tracked at several locations in the image field of view, however the source locations were always static with no movement. This evaluation would allow the assessment and validation of the coregistration without the affects of motion tracking.

After co-registration the phantom was placed in the field of view of the system and rotated by an arbitrary angle around its axis within its case. A digital radiography (DR), single projection image was acquired and the 4 source locations of the phantom were localized by PeTrack simultaneously.

This process was repeated for a total of 5 different phantom rotations while the $\mathrm{C}$-arm gantry was at $0^{\circ}$ (with the $\mathrm{x}$-ray tube directly above the II). The C-arm was rotated to approximately $30^{\circ}$ and the phantom was imaged and tracked at 2 more arbitrary angles. The acquired data consist of 4 tracked locations for each of the 7 projection images.

The tracked source locations were all translated and rotated into the phantom frame using the PeTrack calibration. These 3D points were then projected onto their respective projection images using the $\mathrm{x}$-ray geometric calibration.

The projected source locations were identified and displayed on a projection image acquired at $0^{\circ}$ and another at $30^{\circ}$ of gantry rotation(see Fig. 5.10). The projected source locations are indicated by the center of the white circle. The projection images were already corrected for distortion. 


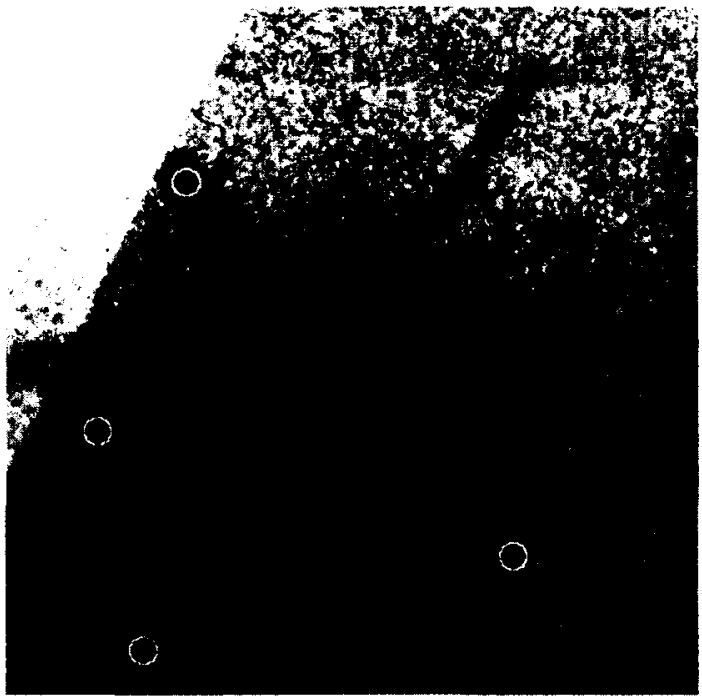

(a) Projection acquired at $0^{\circ}$ of gantry rotation.

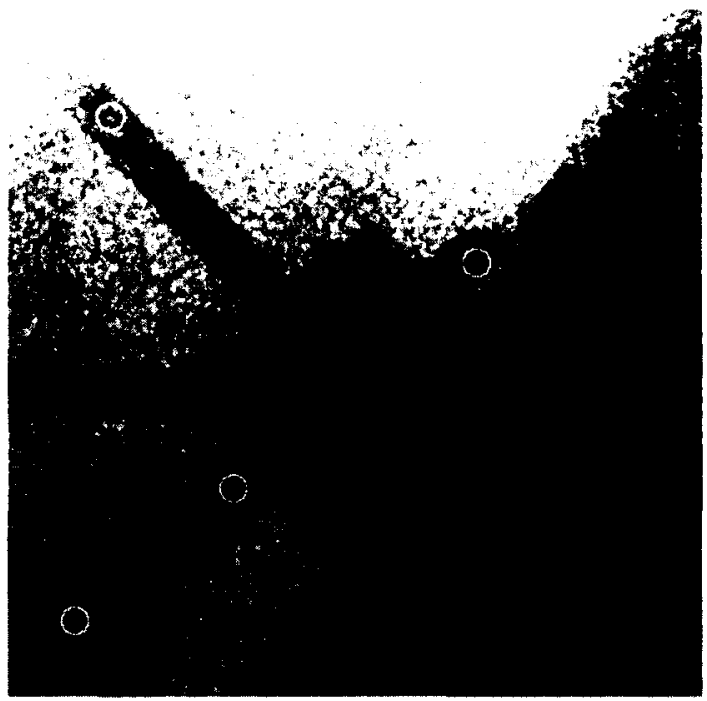

(b) Projection acquired at $30^{\circ}$ of gantry rotation.

Figure 5.10: The projected location of tracked sources onto a projection image. The projected source locations are indicated by the center of the white circle.

In order to perform a quantitative analysis of the co-registration, before obtaining the 7 projection images, two small lead x-ray markers (approximately $0.5 \mathrm{~mm}$ diameter) were glued onto one of the positron sources housings. The lead markers were visually attached to the source housing on opposite sides of the active volume of the source. The x-ray markers can be distinguished on the projection image approximately indicating the source location, see Fig. 5.11a).

To determine the source location in the image using the x-ray markers, the image was magnified, inverted, and the average background value surrounding the x-ray markers was subtracted using ImageJ software [38]. Each x-ray markers location was measured in the image using a center of mass weighting and the source location in the image was assumed to be at the center of the two x-ray markers. The source locations determined from the image and the projected locations of the source are compared in table 5.5 . 


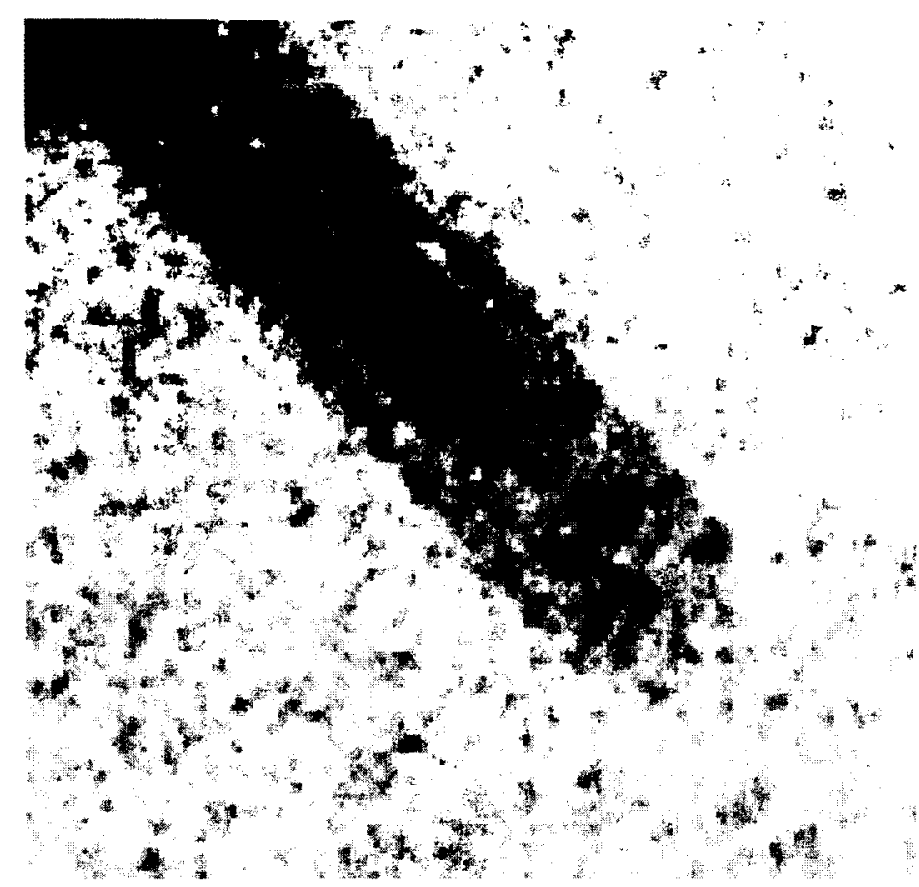

Figure 5.11: Example of $x$-ray markers visually observed on projection image.

The source locations were averaged over several localizations to remove the random uncertainty of the real-time tracking algorithm. The table shows the discrepancy between the projected source location and the location identified on the image of all co-registration trials. It is important to note that the projected source location is greater than the location identified in the image in every observed case suggesting a systematic offset. The average discrepancy and its associated standard deviation is shown. This discrepancy is significant and will be discussed below.

As an additional observation, the PeTrack tracking of the 4 static markers of the phantom at one of the above rotations is shown in table 5.6. The table shows the average and sample standard deviation of $30(x, y, z)$ localizations of the static markers to give an indication of the precision of the PeTrack tracking. Given that the average standard deviation is less then $0.2 \mathrm{~mm}$ it is apparent that the PeTrack tracking precision is fairly good. 
Table 5.5: Comparison between the projected $(u, v)$ source locations (determined from the tracking and co-registration) and the estimated source locations in the image

\begin{tabular}{|c|c|c|c|c|c|c|}
\hline \multirow{2}{*}{$\begin{array}{c}\text { Gantry } \\
\text { angle }\end{array}$} & \multicolumn{2}{|c|}{ Point between centers } & \multicolumn{2}{|c|}{ Projected source location } & \multicolumn{2}{|c|}{ Discrepancy } \\
\hline & $\mathrm{u}$ (pixels) & $\mathrm{v}$ (pixels) & $\mathrm{u}$ (pixels) & $\mathrm{v}($ pixels $)$ & length (pixels) & length (mm) \\
\hline $0^{\circ}$ & 600.0 & 570.5 & 605.8 & 577.0 & 8.7 & 1.8 \\
\hline $0^{\circ}$ & 613.2 & 639.6 & 617.7 & 644.1 & 6.2 & 1.3 \\
\hline $0^{\circ}$ & 618.3 & 657.6 & 622.5 & 660.8 & 5.3 & 1.1 \\
\hline $0^{\circ}$ & 625.7 & 663.6 & 631.9 & 664.5 & 6.2 & 1.3 \\
\hline $0^{\circ}$ & 629.5 & 605.9 & 641.7 & 607.5 & 12.3 & 2.5 \\
\hline $30^{\circ}$ & 597.3 & 440.6 & 602.5 & 444.4 & 6.4 & 1.3 \\
\hline $30^{\circ}$ & 599.3 & 491.6 & 605.0 & 496.6 & 7.6 & 1.6 \\
\hline \multicolumn{5}{|c|}{ Average discrepancy: } & $7.5 \pm 2.4$ & $1.6 \pm 0.5$ \\
\hline
\end{tabular}

Table 5.6: PeTrack tracking precision. The average and standard deviation of 30 measurements of the $(x, y, z)$ location of four tracked sources.

\begin{tabular}{|c|c|c|c|}
\hline Source & $\bar{x} \pm \sigma(\mathrm{mm})$ & $\bar{y} \pm \sigma(\mathrm{mm})$ & $\bar{z} \pm \sigma(\mathrm{mm})$ \\
\hline \hline 1 & $-1.39 \pm 0.15$ & $-17.18 \pm 0.17$ & $23.00 \pm 0.18$ \\
\hline 2 & $4.69 \pm 0.16$ & $-57.55 \pm 0.41$ & $-16.47 \pm 0.24$ \\
\hline 3 & $-2.54 \pm 0.11$ & $-6.79 \pm 0.10$ & $-18.92 \pm 0.22$ \\
\hline 4 & $37.61 \pm 0.08$ & $-25.52 \pm 0.18$ & $4.96 \pm 0.09$ \\
\hline
\end{tabular}




\subsection{Discussion}

The results obtained during the co-registration evaluation did prove the correctness of the technique, though the results obtained were not ideal. The desired accuracy of PeTrack real-time 3D motion tracking is on the order of $0.5 \mathrm{~mm}$. The static coregistration evaluation produced an average discrepancy of $1.6 \pm 0.5 \mathrm{~mm}$. There are several factors that may have attributed to these discrepancies.

The co-registration results relied on the determination of the location of the source on the image by $\mathrm{x}$-ray markers, the accurate PeTrack calibration and the accurate $\mathrm{x}$-ray geometric calibration to project the source locations onto the image. Both calibrations rely on the accuracy of the constructed dual calibration phantom. The observations in table 5.5 show that there is a systematic error on either the projected source locations or the identified source location in the image, or both. The $(u, v)$ locations projected onto the image are always greater than the source location identified in the image. This systematic error must be identified and corrected.

The source location in the image was determined based on very small $\mathrm{x}$-ray markers located in the projection image. The $\mathrm{x}$-ray markers were glued on the source housing at the estimated location of the active source volume. This procedure had an accuracy of $\pm 0.5 \mathrm{~mm}$. Additionally, there would also be error in the determination of the $\mathrm{x}$-ray marker in the image, estimated at approximately \pm 3 pixels, or $\pm 0.62 \mathrm{~mm}$. The combination of these errors would be substantial and a better method for the determination of the source location in the image should be used for evaluation.

The $\mathrm{x}$-ray geometric calibration is known to be very sensitive to the measured and imaged phantom. Small inaccuracies in the determination of the geometric parameters could cause large fluctuations in the projection of a 3D point onto the image and

possibly a systematic error on the projected source locations. The are several factors, 
described in section 3.5, that may cause error on the imaged and measured ball locations of the phantom. The $c$ vector of the vectors $a, b, c$, which are used to project the 3D source locations onto the image, (see Eqn. 3.3) contains very small values which are rounded off during calibration, possibly affecting the results. Two methods could be utilized to further evaluate this x-ray geometric calibration. First, an image reconstruction of the dual calibration phantom could simply attempt to test the calibration parameters. Secondly, the 6 balls of dual calibration could be projected back onto the image after their determination to test the precision of the geometric calibration while removing any inaccuracies due to the PeTrack source localization and system calibration.

The results in table 5.6 show that the precision of the PeTrack localization is good, and the tracked locations do not significantly vary during tracking. Further, section 5.2.3 shows that under ideal conditions the PeTrack calibration performs exceptionally well. In actual operation however the PeTrack system may still have quite substantial systematic errors from imperfect alignment. The PeTrack geometric calibration fits for a translation and rigid rotation are based on many assumptions about the alignment of the detector modules. For example, the pairs of modules are assumed to be oriented at exactly $90^{\circ}$ to one another, each detector module is assumed to have no rotation relative to the field of view and they are assumed to have no lateral shift relative to each other. If any oné of these assumptions is not true due to improper alignment, a systematic error on the localization of tracked sources would be observed.

\subsubsection{Summary}

Despite the observed inadequacies, this chapter described several advancements towards the successful completion of the PeTrack project. The construction and demonstration of a PeTrack system prototype was shown. The first ever positron emission 
detector module geometric calibration technique was designed, validated, and employed. A unique dual x-ray/PeTrack phantom was designed, constructed, and utilized. Finally, the successful co-registration between the x-ray C-arm imaging system and the positron emission tracking system was accomplished. Further investigation is required to determine and correct any systematic errors affecting the co-registration. 


\section{Chapter 6}

\section{Conclusions \& Future Work}

\subsection{Distortion measurement \& correction}

Angle dependent S-distortion was shown to have a significant influence on accurate image acquisition, and distortion correction must be implemented. The necessity and benefits of distortion correction have also been thoroughly demonstrated in chapters $3 \& 4$.

Though the distortion correction procedure presented here is not as rigorous as other methods in the literature, it can correct for angle dependent S-distortion within our desired level of accuracy. If the accuracy of the correction needs improvement, the same distortion correction method could be implemented with a better grid phantom. The grid phantom could be improved by simply quadrupling the number of balls in the grid by essentially halving the center to center distance between balls and quadrupling the number of quadrilateral correction regions.

The process of correcting a single projection image requires only 25 seconds of computation, however, that relates to 42 minutes of computation time for a 100 projection scan. Future work must be done to reduce this correction time to an acceptable level for on-demand correction and reconstruction; $10-30$ fold increase in computation speed is desired. This could possibly be accomplished by both programing 
the correction procedure in a more efficient language as well as using parallel graphic processing cards.

\subsection{Scanner geometry calibration}

A robust and advanced method of geometric calibration was implemented and through successful image reconstruction, was validated for the first time with real data. A simple and unique geometric calibration phantom was designed and constructed for utilization in this calibration. The results of the geometric calibration displayed a fair amount of local fluctuation and global variation of the calibration parameters. Future work should involve an evaluation of the degree of sensitivity to inaccuracies of the imaged calibration ball locations as well as the measured specifications of the calibration phantom. An effective method of accurately measuring the specifications of the geometric calibration phantom should also be investigated.

\subsection{Image reconstruction}

Image reconstruction was an invaluable operation to validate the method of $\mathrm{x}$-ray geometric calibration. It was further useful to evaluate both the geometric calibration and the distortion correction methods and prove their necessity. The image reconstructions showed that correction of S-distortion was essential to obtain the best image quality, a process the C-arm on-board system cannot do. Consequently, improved resolution of reconstructed images has been shown and the image quality desired for accurate co-registration and tracking with PeTrack has been achieved.

Further work should be done to attempt to reduce truncation effects by smoothing projection images around the edge of the field of view. The image quality could also be improved by using a flood field image acquisition to compensate for the detector 
sensitivity variations. Additionally, the construction of a specialized phantom for the testing of CT image quality would be beneficial. Finally, further endeavors should be pursued to increase the speed of the reconstruction process to allow for on demand 3D image reconstruction. The degree to which the computation speed must be increased heavily depends on the desired 3D image quality.

\subsection{PeTrack calibration \& co-registration}

The development and validation of a PeTrack calibration method was accomplished and then utilized for the co-registration between the $\mathrm{x}$-ray imaging and PeTrack tracking systems. This was achieved after the design and construction of a dual $\mathrm{x}$-ray/PeTrack calibration phantom.

The co-registration evaluation exhibited an unaccounted for systematic error. Identification and correction of this source of systematic error is essential. This effect could possibly be attributed to the mis-alignment of the PeTrack system, or inaccuracies in the dual calibration phantom leading to either an erroneous x-ray or PeTrack geometric calibration.

Future efforts must be directed toward the investigation of a better alignment procedure or a more extensive PeTrack calibration method that can measure and correct for the most influential or faulty variables affecting the calibration. Furthermore, it would be beneficial to devise a better way of evaluating the co-registration tracking accuracy. Future evaluations should not require the determination of the location of a source on the image by means of inaccurate $\mathrm{x}$-ray markers. It would also be beneficial to separately evaluate the $\mathrm{x}$-ray geometric calibration and the PeTrack calibration and system alignment to determine the relative effects of the two systems. 


\subsection{Project summary}

The research presented in this project exhibits significant strides towards the completion of a full scale PeTrack prototype which is capable of real-time motion tracking of tools, to allow for precise manipulation and operation, or tumours, as a means of accurate delivery of radiation therapy to tumours undergoing motion due to breathing. There are more improvements which need to be met before this is possible.

In this work positron sources were shown to be tracked by PeTrack and then projected onto a 2D projection image. Though 3D tracking was not shown it can be assumed that if image reconstruction can accurately construct a 3D volume from 2D projection data and the PeTrack source locations can be projected onto those 2D projections, tracked source locations can be accurately projected into a $3 \mathrm{D}$ volume.

This study only demonstrated the static localization and projection of the sources tracked by PeTrack. To extend this to motion tracking would presumably be of little difficulty. Provided the time delay between the imaging and PeTrack tracking of sources was known, motion tracking on an image would only require the continuous projection of the tracked source location onto the image utilizing the $\mathrm{x}$-ray geometric parameters.

Real-time motion tracking, on the other hand, requires the software integration of the PeTrack tracking algorithm, the PeTrack and imaging system calibration methods, and the image acquisition procedure which will be a crucial process to implement in the future.

Once these obstacles have been surpassed only some minor advancements will need to be overcome before the PeTrack system is ready for comprehensive phantom testing, and subsequently clinical implementation. 


\section{List of References}

[1] C. Mennessier, R. Clackdoyle, and F. Noo, "Direct determination of geometric alignment parameters for cone-beam scanners.," Physics in Medicine and Biology, vol. 54, pp. 1633-60, Mar. 2009.

[2] C. Mennessier, B. Spencer, R. Clackdoyle, a C. Conneau, and T. Xu, "Distortion correction, geometric calibration, and volume reconstruction for an isocentric CArm X-Ray system," 2011 IEEE Nuclear Science Symposium Conference Record, pp. 2943-2947, Oct. 2011.

[3] B. Spencer, R. Clackdoyle, C. Mennessier, and T. Xu, "Distortion Correction, Geometric Calibration, and Volume Reconstruction for an Isocentric C-Arm XRay System : Preliminary Studies," 2012 international conference of CT imaging proceeding, 2012.

[4] M. K. Chmarra, C. Grimbergen, and J. Dankelman, "Systems for tracking minimally invasive surgical instruments.," Minimally invasive therapy $\&$ allied technologies : MITAT : official journal of the Society for Minimally Invasive Therapy, vol. 16, pp. 328-40, Jan. 2007.

[5] Z. Dvir and T. Prushansky, "Reproducibility and instrument validity of a new ultrasonography-based system for measuring cervical spine kinematics.," Clinical biomechanics (Bristol, Avon), vol. 15, pp. 658-64, Nov. 2000.

[6] F. Banovac, J. Tang, S. Xu, D. Lindisch, H. Y. Chung, E. B. Levy, T. Chang, M. F. McCullough, Z. Yaniv, B. J. Wood, and K. Cleary, "Precision targeting of liver lesions using a novel electromagnetic navigation device in physiologic phantom and swine," Medical Physics, vol. 32, no. 8, p. 2698, 2005.

[7] J. Hummel, M. Figl, C. Kollmann, H. Bergmann, and W. Birkfellner, "Evaluation of a miniature electromagnetic position tracker," Medical Physics, vol. 29, no. 10, p. $2205,2002$. 
[8] J. B. Hummel, M. R. Bax, M. L. Figl, Y. Kang, C. Maurer, W. W. Birkfellner, H. Bergmann, and R. Shahidi, "Design and application of an assessment protocol for electromagnetic tracking systems," Medical Physics, vol. 32, no. 7, p. 2371, 2005 .

[9] K. Schicho, M. Figl, M. Donat, W. Birkfellner, R. Seemann, A. Wagner, H. Bergmann, and R. Ewers, "Stability of miniature electromagnetic tracking systems.," Physics in medicine and biology, vol. 50, pp. 2089-98, May 2005.

[10] J. D. P. Hoisak, K. E. Sixel, R. Tirona, P. C. F. Cheung, and J.-P. Pignol, "Correlation of lung tumor motion with external surrogate indicators of respiration.," International journal of radiation oncology, biology, physics, vol. 60, pp. 1298-306, Nov. 2004.

[11] D. Ionascu, S. B. Jiang, S. Nishioka, H. Shirato, and R. I. Berbeco, "Internalexternal correlation investigations of respiratory induced motion of lung tumors," Medical Physics, vol. 34, no. 10, p. 3893, 2007.

[12] H. Shirato, S. Shimizu, K. Kitamura, T. Nishioka, K. Kagei, S. Hashimoto, H. Aoyama, T. Kunieda, N. Shinohara, H. Dosaka-Akita, and K. Miyasaka, "Four-dimensional treatment planning and fluoroscopic real-time tumor tracking radiotherapy for moving tumor.," International journal of radiation oncology, biology, physics, vol. 48, pp. 435-42, Sept. 2000.

[13] H. Shirato, S. Shimizu, T. Kunieda, K. Kitamura, M. van Herk, K. Kagei, T. Nishioka, S. Hashimoto, K. Fujita, H. Aoyama, K. Tsuchiya, K. Kudo, and K. Miyasaka, "Physical aspects of a real-time tumor-tracking system for gated radiotherapy.," International journal of radiation oncology, biology, physics, vol. 48, pp. 1187-95, Nov. 2000.

[14] H. Shirato, M. Oita, K. Fujita, Y. Watanabe, and K. Miyasaka, "Feasibility of synchronization of real-time tumor-tracking radiotherapy and intensitymodulated radiotherapy from viewpoint of excessive dose from fluoroscopy.," International journal of radiation oncology, biology, physics, vol. 60, pp. 335-41, Sept. 2004.

[15] J. M. Balter, J. N. Wright, L. J. Newell, B. Friemel, S. Dimmer, Y. Cheng, J. Wong, E. Vertatschitsch, and T. P. Mate, "Accuracy of a wireless localization system for radiotherapy.," International journal of radiation oncology, biology, physics, vol. 61, pp. 933-7, Mar. 2005. 
[16] T. R. Willoughby, P. a. Kupelian, J. Pouliot, K. Shinohara, M. Aubin, M. Roach, L. L. Skrumeda, J. M. Balter, D. W. Litzenberg, S. W. Hadley, J. T. Wei, and H. M. Sandler, "Target localization and real-time tracking using the Calypso 4D localization system in patients with localized prostate cancer.," International journal of radiation oncology, biology, physics, vol. 65, pp. 528-34, June 2006.

[17] U. Topal and B. Ediz, "Transthoracic needle biopsy: factors effecting risk of pneumothorax.," European journal of radiology, vol. 48, pp. 263-7, Dec. 2003.

[18] J. W. Cannon, J. A. Stoll, I. S. Salgo, H. B. Knowles, R. D. Howe, P. E. Dupont, G. R. Marx, and P. J. del Nido, "Real-time three-dimensional ultrasound for guiding surgical tasks.," Computer aided surgery : official journal of the International Society for Computer Aided Surgery, vol. 8, pp. 82-90, Jan. 2003.

[19] T. Shchory, D. Schifter, R. Lichtman, D. Neustadter, and B. W. Corn, "Tracking accuracy of a real-time fiducial tracking system for patient positioning and monitoring in radiation therapy.," International journal of radiation oncology, biology, physics, vol. 78, pp. 1227-34, Nov. 2010.

[20] W. J. M. de Kruijf, J. Verstraete, D. Neustadter, B. W. Corn, S. Hol, J. L. M. Venselaar, R. J. Davits, B. P. Wijsman, L. Van den Bergh, T. Budiharto, R. Oyen, K. Haustermans, and P. M. P. Poortmans, "Patient Positioning Based on a Radioactive Tracer Implanted in Patients With Localized Prostate Cancer: A Performance and Safety Evaluation.," International journal of radiation oncology, biology, physics, pp. 1-6, May 2012.

[21] D. Neustadter, G. Barnea, S. Stokar, and B. Corn, "Analysis of dose to patient, spouse/caretaker, and staff, from an implanted trackable radioactive fiducial for use in the radiation treatment of prostate cancer," Medical Physics, vol. 37, no. 3, p. 1220,2010 .

[22] T. Xu, J. T. Wong, P. M. Shikhaliev, J. L. Ducote, M. S. Al-Ghazi, and S. Molloi, "Real-time tumor tracking using implanted positron emission markers: Concept and simulation study," Medical Physics, vol. 33, no. 7, p. 2598, 2006.

[23] J. T. Bushberg, J. A. Seibert, E. M. L. Jr., and J. M. Boone, The Essential Physics of Medical Imaging (2nd Edition). Lippincott Williams \& Wilkins, 2001.

[24] N. W. Churchill, M. Chamberland, and T. Xu, "Algorithm and simulation for real-time positron emission based tumor tracking using a linear fiducial marker.," Medical physics, vol. 36, pp. 1576-86, May 2009. 
[25] S. Jan, G. Santin, D. Strul, S. Staelens, K. Assié, D. Autret, S. Avner, R. Barbier, M. Bardiès, P. M. Bloomfield, D. Brasse, V. Breton, P. Bruyndonckx, I. Buvat, A. F. Chatziioannou, Y. Choi, Y. H. Chung, C. Comtat, D. Donnarieix, L. Ferrer, S. J. Glick, C. J. Groiselle, D. Guez, P.-F. Honore, S. Kerhoas-Cavata, A. S. Kirov, V. Kohli, M. Koole, M. Krieguer, D. J. van der Laan, F. Lamare, G. Largeron, C. Lartizien, D. Lazaro, M. C. Maas, L. Maigne, F. Mayet, F. Melot, C. Merheb, E. Pennacchio, J. Perez, U. Pietrzyk, F. R. Rannou, M. Rey, D. R. Schaart, C. R. Schmidtlein, L. Simon, T. Y. Song, J.-M. Vieira, D. Visvikis, R. V. de Walle, E. Wieërs, and C. Morel, "GATE: a simulation toolkit for PET and SPECT," Physics in Medicine and Biology, vol. 49, pp. 4543-4561, Oct. 2004.

[26] M. Chamberland, R. Wassenaar, B. Spencer, and T. Xu, "Performance evaluation of real-time motion tracking using positron emission fiducial markers," Medical Physics, vol. 38, no. 2, p. 810, 2011.

[27] M. Chamberland, Performance evaluation and algorithm development for realtime tumour tracking using positron emi ... Msc thesis, Carleton University, 2009 .

[28] M. Soleimani, Detector development for positron emission based real-time tumor tracking. Msc thesis, Carleton University, 2009.

[29] T. Nakamoto, Y. Fukazawa, T. Ohsugi, T. Kamae, and J. Kataoka, "BGO readout with photodiodes as a soft gamma-ray detector at," Nuclear Instruments and Methods in Physics Research Section A: Accelerators, Spectrometers, Detectors and Associated Equipment, vol. 536, pp. 136-145, Jan. 2005.

[30] mesytec GmbH \& co KG, "MPET: Readout System for Position Sesnitive PMTs, Coincidence Timing - Users Manual," 2007.

[31] Siemens Medical, "Enhanced precision in the OR," 2007.

[32] S. Rudin, D. Bednarek, and R. Wong, "Accurate characterization of image intensifier distortion," Medical physics, 1991.

[33] E. Pietka and H. Huang, "Correction of aberration in image-intensifier systems," Computerized medical imaging and graphics, vol. 16, no. 4, pp. 253-258, 1992.

[34] R. Fahrig, M. Moreau, and D. W. Holdsworth, "Three-dimensional computed tomographic reconstruction using a C-arm mounted XRII: correction of image intensifier distortion.," Medical Physics, vol. 24, pp. 1097-106, July 1997. 
[35] R. R. Liu, S. Rudin, and D. R. Bednarek, "Super-global distortion correction for a rotational C-arm x-ray image intensifier," Medical Physics, vol. 26, pp. 180210, Sept. 1999.

[36] B. Schueler and X. Hu, "Correction of image intensifier distortion for threedimensional x-ray angiography," Proceedings of SPIE, vol. 2432, pp. 272-913, 1995.

[37] X. Wang and R. Ning, "Accurate and efficient image intensifier distortion correction algorithm for volume tomographic angiography," Optical engineering, no. May 2011, p. 1802, 1998.

[38] S. J. Abramoff, Magalhães, Paulo J., Ram, "Image processing with ImageJ," Biophotonics international, vol. 11, no. 7, pp. 36-42, 2004.

[39] R. Fahrig and D. W. Holdsworth, "Three-dimensional computed tomographic reconstruction using a C-arm mounted XRII: image-based correction of gantry motion nonidealities.," Medical Physics, vol. 27, pp. 30-8, Jan. 2000.

[40] N. Strobel, B. Heigl, T. Brunner, and O. Schuetz, "Improving 3D image quality of x-ray C-arm imaging systems by using properly designed pose determination systems for calibrating the projection," Proceedings of SPIE, vol. 5030, no. 2003, pp. 943-954, 2003.

[41] R. Clackdoyle and C. Mennessier, "Centers and centroids of the cone-beam projection of a ball.," Physics in medicine and biology, vol. 56, pp. 7371-91, Dec. 2011.

[42] A. Kak and M. Slaney, "Algorithms for Reconstruction with Nondiffracting Sources," in Computerized tomographic imaging, no. 1, IEEE Press., 1999.

[43] L. Feldkamp and L. Davis, "Practical cone-beam algorithm," JOSA A, vol. 1, no. 6 , pp. 612-619, 1984.

[44] D. L. Parker, "Optimal short scan convolution reconstruction for fanbeam CT.," Medical physics, vol. 9, no. 2, pp. 254-7, 1982.

[45] C. Mennessier and R. Clackdoyle, "Automated geometric calibration and reconstruction in circular cone-beam tomography," 2008 IEEE Nuclear Science Symposium Conference Record, pp. 5081-5085, Oct. 2008. 
[46] M. J. Daly, J. H. Siewerdsen, Y. B. Cho, D. Jaffray, and J. C. Irish, "Geometric calibration of a mobile C-arm for intraoperative cone-beam CT," Medical Physics, vol. 35, no. 5, p. 2124, 2008. 